UNIVERSIDADE DE SÃO PAULO

INSTITUTO DE QUÍMICA DE SÃO CARLOS

DEPARTAMENTO DE FÍSICO-QUÍMICA

\title{
Instabilidades Cinéticas em Sistemas Eletroquímicos: Uma Contribuição Teórica
}


Melke Augusto do Nascimento

\section{Instabilidades Cinéticas em Sistemas Eletroquímicos: Uma Contribuição Teórica}

Tese apresentada ao Instituto de Química de São Carlos para obtenção do título de Doutor em Química com ênfase em Físico Química

Orientador: Prof. Dr. Hamilton Varela

São Carlos - SP 
À memória de meu pai, João Augusto do Nascimento pelos ensinamentos ao longo de minha vida;

À minha querida mãe, Iracema Coelho pelo amor $e$ carinho para com seu filho;

À minha amada esposa, Lucélia Cristina pelo amor e apoio nas horas boas e ruins;

E ao meu filho querido, João Augusto por ser luz na minha vida. 


\section{Agradecimentos}

Ao Prof. Dr. Hamilton Varela pela amizade, orientação e disposição nas discussões durante todo o meu doutorado, sempre fazendo questionamentos importantes que levaram à reflexão do trabalho científico;

Ao Prof. Dr. Jason Gallas pela discussão enriquecedora referente a este trabalho;

Ao Prof. Dr. Markus Eiswirth pela oportunidade de poder ajudar com o trabalho referente ao modelo teórico do metanol;

Ao Prof. Dr. Punit Parmananda que durante os dez dias em que esteve no Brasil sempre esteve conversando e dando idéias interessantes relacionadas às modelagens de sistemas dinâmicos espacialmente estendidos;

Aos colegas do laboratório Elton, Eduardo, Nickson, Murilo e Cristiane que sempre estavam presente para tirar algumas dúvidas com relação aos experimentos e pelas conversas na hora do café, sempre com muita descontração. Em especial gostaria de agradecer ao Bruno Batista, Emerson Boscheto e Raphael Nagao pelas discussões relacionada a ciência, filosofia e arte. Gostaria de agradecer também ao Eduardo Ciapina, Manuel Farias, Leandro Gurgel, Luciano dos Santos e Roberto Lima;

Por fim, agradeço à Fundação de Amparo à Pesquisa no Estado de São Paulo (FAPESP) pela aprovação do projeto $(07 / 04522$ - 0) sem a qual não teria condição de realizar esse trabalho. Gostaria de agradecer também ao Instituto de Química de São Carlos (IQSC) pela excelente estrutura que possibilitou saciar um pouco minha curiosidade. 
Tem um sentido a minha vida? A vida de um homem tem sentido? Posso responder a tais perguntas se tenho espírito religioso. Mas, "fazer tais perguntas tem sentido?" Respondo: "Aquele que considera sua vida e a dos outros sem qualquer sentido é fundamentalmente infeliz, pois não tem motivo algum para viver".

Albert Einstein em Como vejo o mundo

Não basta ensinar ao homem uma especialidade. Porque se tornará assim uma máquina utilizável, mas não uma personalidade. É necessário que adquira um sentimento, um senso prático daquilo que vale a pena ser empreendido, daquilo que é belo, do que é moralmente correto. A não ser assim, ele se assemelhará, com seus conhecimentos profissionais, mais a um cão ensinado do que a uma criatura harmoniosamente desenvolvida. Deve aprender a compreender as motivações dos homens, suas quimeras $e$ suas angústias para determinar com exatidão seu lugar exato em relação a seus próximos e à comunidade.

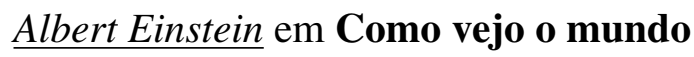




\section{Resumo}

Nascimento, M. A. Instabilidades Cinéticas em Sistemas Eletroquímicos: Uma Contribuição Teórica. 2011. Tese (Doutorado) - Instituto de Química de São Carlos, Universidade de São Paulo, São Carlos, 2011.

Mais que fenômenos exóticos, oscilações de corrente e potencial são bastante comuns em vários sistemas eletroquímicos. Ainda que conhecidos há muito tempo, processos oscilatórios na interface sólido/líquido eletrificada são relativamente pouco investigados sob o ponto de vista teórico. São apresentados nessa Tese dois trabalhos, o primeiro relacionado às instabilidades cinéticas observadas em tais sistemas, por meio de um modelo formado por três equações diferenciais não-lineares ordinárias acopladas, que representam um protótipo mínimo do comportamento complexo observado em reações eletrocatalíticas. Especificamente, este protótipo reproduz as características gerais de osciladores eletroquímicos caracterizados por uma resistência diferencial negativa parcialmente escondida em uma curva de corrente/potencial em forma de $\mathrm{N}$. O modelo foi abordado utilizando as análises convencionais e os diagramas de estabilidade, de Lyapunov e de período. A partir dos diagramas de estabilidade foi possível descrever o comportamento do sistema levando em consideração a condição homoclínica de Shilnikov. Já os diagramas de Lyapunov e período mostraram de forma detalhada o comportamento caótico e periódico do modelo, em que se pode observar a existência de estruturas auto-organizadas nos domínios de periodicidade em um fundo caótico, onde tais estruturas são chamadas de shrimps. A observação de tais estruturas que também são encontradas em outros sistemas reforçando a hipótese da universalidade estrutural para fenômenos de codimensão dois. A segunda parte dessa Tese consiste num estudo do drift observado em séries experimentais aplicando técnicas de análise multivariada a uma série temporal experimental obtida para eletro-oxidação da molécula do metanol em Pt policristalina. O resultado mostrou que podemos descrever a influência do drift no comportamento oscilatório por meio de três variáveis relacionados aos processos superficiais.

Palavras chaves: Modelo eletroquímico; órbitas homoclínicas; expoente de Lyapunov; análise multivariada; série temporal. 


\section{Abstract}

Nascimento, M. A. Kinetic Instabilities in Electrochemical Systems: A Theoretical Contribution. 2011. Tese (Doutorado) - Instituto de Química de São Carlos, Universidade de São Paulo, São Carlos, 2011.

More than just an exotic phenomenon, oscillations of potential and current are often found in several electrochemical systems. Although oscillatory processes at solid/liquid electrified interfaces have been reported a long time ago, just few theoretical studies have been done so far. This Thesis comprises two parts: the first one analyzes kinetic instabilities observed in electrochemical systems by using a model consisting of three non-linear coupled ordinary differential equations that represent a prototype of the complex behavior observed in electrocatalytic systems. Specifically, this prototype captures the general characteristics of electrochemical oscillators that display a negative differential resistance partially hidden for an $\mathrm{N}$-shaped current/potential curve. The model was studied using conventional analyses and stability diagrams, Lyapunov exponents and the evaluation of the period of oscillations. From the stability diagrams it was possible to describe the behavior of the system taking into consideration the homoclinic Shilnikov condition. The Lyapunov and period analyses showed in a very detailed manner the chaotic and periodic behavior of the model, where it is observed the existence of self-organized structures in the domains of periodicity on a chaotic background. Those structures are known as shrimps. The observation of such structures that are also found in other systems reinforces the idea of structural universality for codimension two phenomena. The second part of the Thesis deals with the analysis of the oscillatory drift by using multivariate analysis techniques to an experimental time series obtained for the electroxidation of methanol on polycrystalline $P t$. The results showed that it is possible to describe the influence of the

drift during the oscillatory behavior by means of three variables that act on the surface of the electrode.

Keywords: Electrochemical model; homoclinics orbtis; Lyapunov's exponent; multivariate analysis; time series. 


\section{Lista de Figuras}

1 Diagrama de órbitas para o mapa logístico com o parâmetro $\alpha$ variando discretamente. . . . . . . . . . . . . . . . . . 21

2 Esquema generalizado da interação entre os ciclos de retro-alimentação positivo e negativo. . . . . . . . . . . . . . . . . . . . 23

3 Circuito equivalente referente a uma interface eletrificada sob controle potenciostático.

4 (a) curva no estado estacionário para $I$ versus $\phi$, (b) diagrama de bifurcação para $I$ versus o parâmetro $U$ e (c) diagrama de bifurcação sela-nó para o espaço

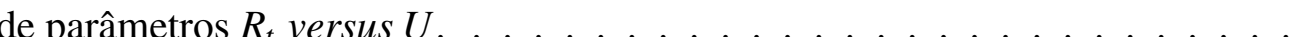

5 Esquema ilustrativo da variação de concentração próximo ao eletrodo no inter-

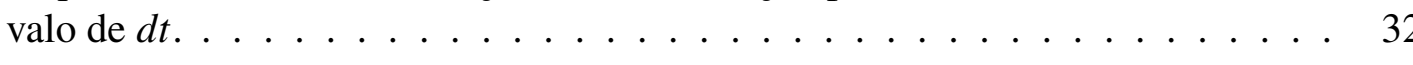

6 (a) curva no estado estacionário para $I$ versus $\phi$ e (b) diagrama de bifurcação sela-nó para o espaço de parâmetros $\rho^{\prime}$ versus $U$.

7 Curva estacionária para o modelo $H N-N D R$ no espaço de fase $I$ versus $\phi$. A linha vermelha tracejada corresponde à região instável do modelo.

8 Perfis estacionários das contribuições para o modelo constituído pelas Equações 19, 20 e 21, onde a linha preta corresponde ao perfil $N-N D R$, linha vermelha ao recobrimento da espécie bloqueante e a linha azul o comportamento observado $H N-N D R . \ldots \ldots \ldots \ldots \ldots \ldots \ldots \ldots$

9 (a) e (c) correspondem às séries temporais periótica e cáotica para as condições de $\varepsilon=0,001, \mu=50$ e $\rho=106,7$ com dois valores distintos de $U=244$ e $U=246$. As figuras (b) e (d) são os respectivos atratores periódico e cáotico de (a) e $($ b) . . . . . . . . . . . . . . . . . . . . . . .

10 Diagramas de bifurcação referente aos perfis de $I_{F}$ versus $U$ para valores de $\rho$ (a) 30 , (b) 50 e (c) 180 com $\varepsilon=0,001$ e $\mu=50$.

11 Diagramas de orbitas refrente a $\rho=180$, onde (a) corresponde ao diagrama na faixa de $200<U<500$ e (b) $288.5<U<289.3 \ldots \ldots \ldots$. . . . . . . . . 50

12 Curvas de bifurcação no plano de parâmetros $\rho$ versus $U$ para $\varepsilon=0,001$ e $\mu=50.51$ 
13 Esboço das possíveis trajetórias adotadas pelos pontos fixos no espaço de fase tridimensional. Nesse esboço são mostradas duas possíveis configurações para os pontos de sela-nó (c) tipo I e (d) tipo II, assim como os pontos de sela-foco (g) tipo I e (h) tipo II. . . . . . . . . . . . . . . . . . . . . . . . .

14 Diagramas de bifurcação 3D para (a) plano de variáveis e parâmetro $(\phi, c, \rho) \mathrm{e}$ (b) plano de parâmetros e variável $(\rho, U, c)$, nas condições de $\varepsilon=0,001$ e $\mu=50.56$

15 Curvas estacionárias para os valore de (a) $\rho=100$ e (b) $\rho=280$ caculadas para os valores de $\varepsilon=0,001$ e $\mu=50$. As séries temporais referentes às órbitas $P_{A 1}$, $P_{A 2}, P_{B 1}$ e $P_{B 2}$ são mostradas em (a2), (a3), (b2) e (b3) respactivamente. . . . . .

16 Órbitas nas condições de Hopf, sela-nó I e II calculadas para o parâmetro $\rho=$ $280, \varepsilon=0,001$ e $\mu=50$, onde (a) e (b) correspondem às órbitas referentes aos pontos de Hopf no plano de fase $(\phi, c)$ e $(\phi, \theta)$. Já (c) e (d) referem-se às órbitas dos pontos de sela-nó no plano de fase $(\phi, c)$ e $(\phi, \theta) \ldots \ldots \ldots$. . . . .

17 Diagrama de estabilidade pontual para (a) espaço de parâmetro $\rho$ versus $U$ e (b) diagrama de estabilidade pontual deformado em relação ao eixo $U$, obtidos para os valores de $\varepsilon=0,001$ e $\mu=50 \ldots \ldots \ldots \ldots$

18 Diagramas de bifurcações de Hopf no espaço $\rho$ versus $U$ comparando a infuência dos parâmetros (a) $\varepsilon$ mantendo constante o valor de $\mu=50$ e (c) $\mu$ mantendo o parâmetro $\varepsilon$ constante e igual a 0,001 . Já os gráficos (b) e (d) correspondem às curvas das intensidade normalizada para $\phi_{N}, c_{N}, \theta_{N}$ e $I_{F N}$ quando variamos os parâmetros $\varepsilon$ e $\mu$ nas condições de $\rho=50, U=250$ para (b) e $\rho=55$, $U=250,2$ para $(\mathrm{d}) \ldots \ldots \ldots \ldots \ldots \ldots$

19 Estabilidades pontuais para o espaço de parâmetros $\rho$ versus $U$ calculados para o valore de $\varepsilon=0,005$ e $\mu=50$, onde (a) corresponde ao diagrama com o parâmetro $U$ corrigido e (b) sem a correção. . . . . . . . . . . . . . .

20 Órbitas nas condições de Hopf, Sela-nó I e II calculadas para o parâmetro $\rho=$ 280, $\varepsilon=0,005$ e $\mu=50$, onde (a) corresponde a curva estacionária no plano de fase $(\phi, c)$ e $(b)$ referente ao plano $(\phi, \theta) \ldots \ldots \ldots$

21 Diagramas de estabilidades pontuais no espaço de parâmetros $\rho$ versus $U$ com uma variação de $\varepsilon$ dada por (a) 0,0005, (b) 0,004, (c) 0,006 e (d) 0,01, man-

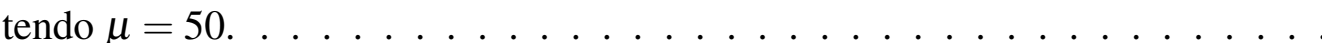

22 Diagramas de Lyapunov calculados para $\varepsilon=0,001$ e $\mu=50$ no plano de parâmetro $\rho$ versus $U$, onde a Figura 22a corresponde a uma resolução de 700x700, já os gráficos (b), (c), (d) e (e) corresponde a uma resolução de 500x500. 71

23 Diagramas de Lyapunov para $\varepsilon=0,005$ e $\mu=50$ no plano $\rho$ versus $U$, onde o gráfico (a) possui uma resolução de $700 \times 700$. Os gráficos (b) e (c) correspondem as áreas $\mathrm{A}$ e $\mathrm{B}$, já o gráfico (d) corresponde a área $\mathrm{C}$ do gráfico (c), todos com resolução de $500 x 500 \ldots \ldots \ldots \ldots$ 
24 Diagramas de Lyapunov com resolução de $700 \times 700$ no espaço de parâmetros $\rho$ versus $U$ para o sistema $H N-N D R$ em modo potenciostatico com o parâmetro $\varepsilon$ variando de (a) 0,0005, (b) 0,004, (c) 0,006 e (d) 0,01 mantendo o parâmetro

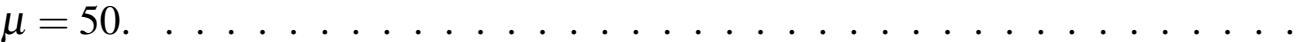

25 Diagramas de período obtidos para o espaço de parâmetros $\rho$ versus $U \operatorname{com} \varepsilon=$ 0,001 e $\mu=50$, onde a escala de cor indica o período entre 1 e 11 . Os períodos acima de 11 estão indicados pela cor preta. A Figura 25 (a) corresponde a uma resolução de 700x700, já os gráficos (b), (c), (d), e (e) possuem uma resolução

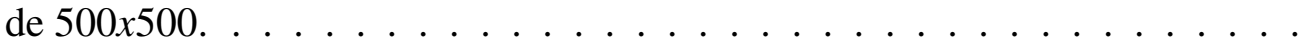

26 Diagrama de órbitas para a linha vermelha tracejada indicada na Figura 25 (a) referente ao valor de $U=280 \operatorname{com} \rho$ variando. O gráfico (b) corresponde a uma ampliação da região interna ao retângulo roxo em (a). As linhas azuis e vermelhas representam as sequências $A$ e $B$ respectivamente. . . . . . . . . . .

27 Esquema mostrando a distribuição dos período para a árvore de (a) Farey e (b) Stern-Brocot. . . . . . . . . . . . . . . . . . . . 79

28 Diagramas de órbitas para as condições referente às linhas (a) $F$ calculada para $\rho=106,63$ e (b) $H$ correspondente variação de $U$ e $\rho$ dada pela Equação 39. As linhas continuas e tracejadas nas figuras indicam o período referentes aos pontos roxos na Figura 25 (e).

29 (a) Série temporal para a eletro-oxidação do metanol à $0,5 \mathrm{ml} \mathrm{L}^{-1}$ obtida para a densidade de corrente $1,00 \mathrm{~mA} \mathrm{~cm}^{-2}$, onde (b) e (c) correspondem às extremidades da região utilizada na análise multivariada. . . . . . . . . . . . . . . . .

30 Função de informação mútua para a série temporal selecionada na Figura 29 (a), onde a seta em vermelho indica a posição do primeiro mínimo da função e o valor do $\tau_{\text {ideal }}=4$

31 Dimensão ED para a série temporal selecionada na Figura 29 (a). . . . . . . . . 91

32 (a) Espaço reconstruído pelo método de Takens com $\tau_{\text {ideal }}=4$ e (b) Matriz de monitoramento obtida pelo método $P S W$ com dimensão incorporada de $d=3$.

33 (a) Gráfico dos autovalores em função do números de fatores na matriz M. Já (b) corresponde ao gráfico da função IND definida por Malinowski [103].

34 Autovetores correspondentes à matriz $\mathbf{M}$, onde (a) e (c) correspondem às matrizes $\mathbf{B}$ e $\mathbf{C}$ sem rotação no espaço de variáveis. Já os gráficos (b) e (d) referemse às matrizes $\mathbf{B}$ e $\mathbf{C}$ rotacionadas no espaço por meio da matriz de rotação $\mathbf{T}$. 


\section{Lista de Tabelas}

1 Relação dos comportamentos dinâmicos esperados em função dos sinais e intensidades dos expoentes de Lyapunov. . . . . . . . . . . . . . . . . . 43

2 Método proposto escrito em pseudocódigo para estimar os períodos no interior de uma bifurcação. . . . . . . . . . . . . . . . . . . . . . . . . . . . 4 44

3 Condições para construção das órbitas referente a Figura 15. . . . . . . . . . . 57

4 Condições para construção das oito órbitas referente às Figuras 16 (a), (b), (c) e (d), onde as órbitas para os pontos de Hopf correspondem a Tabela (a). Já as órbitas de sela-nó do tipo I e II Tabela (b). . . . . . . . . . . . . . . . . . . 60

5 Condições para construção das três órbitas mostradas nas Figuras 20 (a) e (b), as órbitas para o ponto de sela-nó I, sela-nó II e Hopf, referem-se as condições limite da região de instabilidade localizada a direita das curvas de estabilidade.

6 Parâmetros estatísticos obtidos para autovalores da matriz $\mathbf{M}$ 


\title{
Lista de Abreviaturas
}

\author{
NDR Resistência Diferencial Negativa \\ HN-NDR Resistência Diferencial Negativa Parcialmente Escondida \\ PSW Espaço de Fase Alternado \\ IND Função Indicadora de Fator \\ SVD Decomposição dos Valores Singulares \\ REV Erro Reduzido do Autovalor \\ RE Erro Real \\ NS Nível de Significância \\ VA Variância Acumulada \\ MCR Resolução de Curva Multivariada
}




\section{Lista de Símbolos}

$\delta \quad$ Coeficiente de difusão

$\varepsilon \quad$ Parâmetro de controle da escala de tempo do potencial (HN-NDR complexo)

$\varepsilon^{\prime \prime} \quad$ Escala de tempo do potencial (HN-NDR simplificado)

$\varepsilon^{\prime} \quad$ Parâmetro de controle da escala de tempo do potencial (N-NDR)

$\mu \quad$ Parâmetro de controle da escala de tempo da variação de concentração

$\phi \quad$ Potencial da dupla camada

$\phi_{e e} \quad$ Estado estacionário para o potencial da dupla camada

$\rho \quad$ Resistência adimensional (HN-NDR complexo)

$\rho^{\prime \prime} \quad$ Resistência adimensional (HN-NDR simplificado)

$\rho^{\prime} \quad$ Resistência adimensional (N-NDR)

$\theta \quad$ Recoprimento de uma espécie

$\theta_{0}(\phi)$ Constante de recobrimento

$\theta_{n} \quad$ Nullcline para o recobrimento

$\theta_{e e} \quad$ Estado estacionário para o recobrimento

A Área do eletrodo

c Concentração de uma espécie

$c^{b} \quad$ Concentração no volume do eletrólito

$c_{0} \quad$ Concentração próxima à superfície do eletrodo

$c_{n} \quad$ Nullcline para concentração

$C_{d l} \quad$ Capacitância da dupla camada elétrica

$c_{e e} \quad$ Estado estacionário para a concentração

$D \quad$ Coeficiente de difusão

F $\quad$ Constante de Faraday

I Corrente total 
$I_{C} \quad$ Corrente capacitiva

$I_{F}(\phi)$ Corrente fadaica

$k(\phi)$ Constante cinética da reação

$n \quad$ Número de elétrons envolvidos na reação

$R_{\Omega} \quad$ Resistência da suloção

$R_{\text {ext }} \quad$ Resistência externa

$R_{t} \quad$ Resistência total

U Potencial aplicado

$Z_{F} \quad$ Impedância fadaraica 


\section{Sumário}

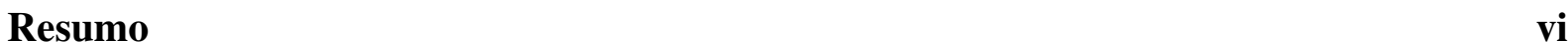

Abstract $\quad$ vii

Lista de Figuras $\quad$ viii

Lista de Tabelas $\quad$ xi

Lista de Abreviaturas $\quad$ xii

Lista de Símbolos $\quad$ xiii

$\begin{array}{ll}\text { Introdução Geral } & 17\end{array}$

Parte I: Dinâmica Temporal de um Modelo Eletrquímico Genérico 18

I.1 Introdução 19

I.1.1 Instabilidades Cinéticas em Sistemas Eletroquímicos . . . . . . . . . . . . 21

I.1.2 Protótipos da Dinâmica Temporal . . . . . . . . . . . . . . . . 24

I.1.2.1 Modelo N-NDR . . . . . . . . . . . . . . . . . . . . 25

I.1.2.1.1 Biestabilidade em Sistemas N-NDR . . . . . . . . . . . . 29

I.1.2.1.2 Oscilações em Sistemas N-NDR . . . . . . . . . . 31

I.1.2.2 Modelo HN-NDR . . . . . . . . . . . . . . . . . . 34

I.1.2.2.1 Comportamento Oscilatório para o Modelo HN-NDR Simples 34

I.1.2.2.2 Modelo HN-NDR Complexo . . . . . . . . . . . . 36

$\begin{array}{llr}\text { I.2 Objetivos } & 39\end{array}$

I.3 Descrição das Simulações $\quad 40$

I.3.1 Diagramas de Bifurcação . . . . . . . . . . . . . . . . . . . . . . 41

I.3.2 Diagramas de Lyapunov . . . . . . . . . . . . . . . . . . . . 42

I.3.3 Diagramas de Período . . . . . . . . . . . . . . . . . . . . . 44

I.4 Resultados e Discussão 46

I.4.1 Análise do Modelo Temporal _. . . . . . . . . . . . . . . . . . 46

I.4.1.1 Discussão Geral do Modelo . . . . . . . . . . . . . . . . . . . . . . 46

I.4.1.2 Diagramas de Bifurcações . . . . . . . . . . . . . . . . . . . 48

I.4.2 Diagramas de Lyapunov . . . . . . . . . . . . . . . . . . . . . . 70 
I.4.3 Diagramas de Período . . . . . . . . . . . . . . . . . . 76

$\begin{array}{lll}\text { I.5 Conclusões } & 82\end{array}$

Parte II: Análise Multivariada Aplicada a Séries Temporais

Experimentais $\quad 83$

$\begin{array}{llr}\text { II.1 Introdução } & 84\end{array}$

$\begin{array}{llr}\text { II.2 } & \text { Objetivos } & 86\end{array}$

II.3 Descrição da Metodologia $\quad 87$

II.3.1 Obtenção da Série Temporal _ . . . . . . . . . . . . . . . . . . . . . 87

II.3.2 Tratamento da Série Temporal . . . . . . . . . . . . . . . . . 87

$\begin{array}{llr}\text { II.4 } & \text { Resultados e Discussão } & 89\end{array}$

$\begin{array}{lll}\text { II.5 Conclusões } & 98\end{array}$

Conclusões Gerais $\quad 99$

$\begin{array}{ll}\text { Perspectivas } & 100\end{array}$

$\begin{array}{ll}\text { Referências Bibliográficas } & 102\end{array}$

$\begin{array}{ll}\text { Curriculum Vitae } & 111\end{array}$ 


\section{Introdução Geral}

O entendimento do comportamento complexo manifestado pela natureza sempre proporcionou um desafio ao intelecto humano. Compreender fenômenos como, padrões nas pelagens de alguns animais, ritmos biológicos e comportamento social, tem de certa forma intro-

duzido importantes questões científicas. É interessante notar que alguns fenômenos complexos podem ser descritos por simples equações diferenciais não-lineares no espaço-tempo, assim como leis organizadas na forma de um autômato celular ou ainda como uma sequência numérica do tipo Farey. Muitos destes fenômenos também estão relacionados ao comportamento de alguns sistemas químicos. Entre as áreas de concentração do conhecimento científico, a química, em especial a eletroquímica, tem sido privilegiada pela sua abrangência tanto no campo experimental com teórico. Nessa Tese será apresentado um estudo constituído por duas partes, uma primeira referente à investigação teórica de um modelo eletroquímico formado por três equações diferenciais não-lineares ordinárias acopladas, que descreve o comportamento complexo observado durante a eletro-oxidação de várias moléculas orgânicas pequenas. Já a segunda parte dessa Tese será constituída por um estudo estatístico de uma série temporal experimental para eletro-oxidação do metanol. A idéia dessa análise é mostrar que pode ser feito um tratamento mais adequado aos dados experimentais de forma a poder inferir com segurança estatística sobre os modelos mecanísticos. 


\section{Parte I}

\section{Dinâmica Temporal de um Modelo Eletroquímico Genérico}




\section{I.1 Introdução}

Sistemas químicos afastados do estado de equilíbrio termodinâmico podem apresentar uma grande variedade de comportamento complexo dito auto-organizado [1, 2]. Tal comportamento, pode ser descrito por um conjunto de equações que descreve a evolução não-linear no tempo e no espaço de forma apropriada. Em sistemas afastados do equilíbrio, são exemplos de comportamento complexo biestabilidade, multiestabilidade, dinâmica oscilatória e formação de padrões espaço-temporais. A forma mais comumente observada em experimentos de laboratório consiste das oscilações temporais, presentes em muitos campos da cinética química, como exemplo podemos citar os trabalhos de Epstein e colaboradores, referente às oscilações de BR (Briggs-Rouscher) e BZ (Belousov-Zhabotinsky) [3-5]. O estudo desses sistemas tem revelado uma grande variedade de comportamentos complexos, incluindo sequências de oscilações simples, de modo misto e caóticas [6, 7].

Os avanços na teoria dos sistemas dinâmicos não-lineares $[1,8]$ têm possibilitado o aprofundamento no entendimento dos processos relacionados a tais sistemas. As propriedades emergentes observadas nesses sistemas dependem diretamente da (a) dinâmica temporal dos elementos reacionais e (b) das interações ou formas de acoplamentos entre esses elementos. Como exemplo das diversas formas apresentadas na dinâmica temporal e acoplamento, podemos citar o trabalho de Bassett e Hudson [9], que relaciona o comportamento dinâmico da eletrodissolução do cobre em solução de ácido clorídrico e os trabalhos de Schell e Albahadily [10, 11] em ácido fosfórico.

Um fato a ser destacado no estudo de sistemas dinâmicos é que a maioria dos fenômenos físicos, químicos e eletroquímicos observados, podem ser modelados por equações diferenciais. Essa descrição matemática possibilita a manipulação de parâmetros vitais no entendimento físico de um sistema dinâmico, que dependendo dos valores assumidos, podem exibir diferentes respostas, como por exemplo pontos fixos estáveis e instáveis, assim como oscilações 
periódicas e caóticas. Essas variações causam uma mudança qualitativa no fluxo ${ }^{\dagger}$ de soluções para o sistema estudado, nesse caso pontos fixos podem ser criados ou destruídos causando uma variação na estabilidade. Tal variação recebe o nome de bifurcação e o valor do parâmetro em que ela ocorre é chamado de ponto de bifurcação.

As mudanças no espaço de fase são discutidas em diversos livros que tratam de sistemas dinâmicos [12-14] que podem ser classificados como sistema contínuos ou discretos. Sistemas contínuos são governados por uma ou mais equações diferenciais ordinárias ou parciais. Já os sistemas discretos têm sua evolução, no tempo discreto, governada por uma ou mais equações de diferenças finitas. Como exemplo de um diagrama que mostra a variação do comportamento dinâmico obtido para um sistema discreto, podemos ver o gráfico na Figura 1, obtido para o modelo do mapa logístico quadrático definido pela Equação 1.

$$
X_{n+1}=\alpha X_{n}\left(1-X_{n}\right)
$$

A Equação logística é utilizada na descrição de populações biológicas, e $X_{n}$ corresponde à variável que representa uma população biológica e $\alpha$ o parâmetro de controle [15].

Observando a Figura 1 que corresponde ao diagrama de órbitas ${ }^{\ddagger}$ para o mapa logístico, Equação 1, percebe-se que a complexidade é manifestada quando se varia o parâmetro de controle. Note que para os valores de $\alpha \leq 3$ a população evolui em direção a um único ponto de equilíbrio estável. Já em $\alpha=3$ há uma bifurcação por uma duplicação de período e um ciclo limite atrativo de período 2 é formado. Na medida em que o parâmetro $\alpha$ é variado, surge uma sequência de bifurcações de duplicação de período até o valor $\alpha=3.5699$, onde o sistema passa por órbitas não-períodicas, sendo que, acima desse valor temos regiões caóticas alternando entre regiões períodico. Este exemplo é deveras interessante, pois mostra claramente como uma simples equação pode representar uma gama enorme de comportamentos dinâmicos.

\footnotetext{
${ }^{\dagger}$ A palavra "fluxo" empregada ao longo desse texto, refere-se ao conjunto de trajetórias obtidas pelas soluções dos sistemas de equações diferenciais e correspondem às órbitas no espaço de fase [14].

‡Os diagrams de órbitas mostram as órbitas dos atratores em função do parâmetro. Já os diagramas de bifurcações mostram, além das órbitas, as regiões de instabilidades [13]. Ambos os diagramas podem ser utilizados para exibir o comportamento interno da região instável.
} 


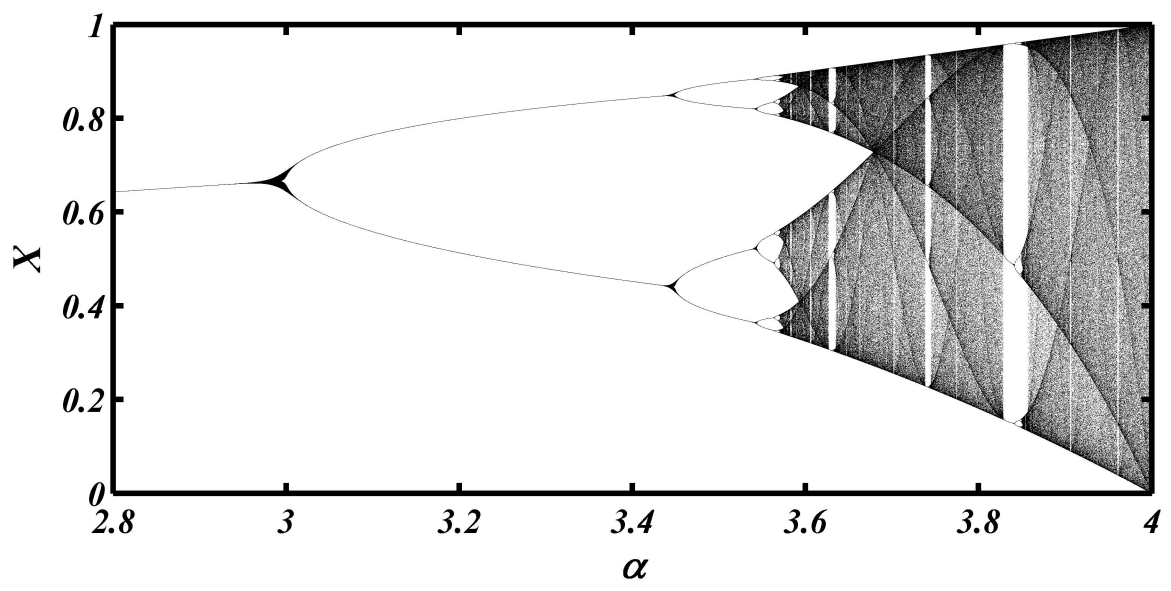

Figura 1: Diagrama de órbitas para o mapa logístico com o parâmetro $\alpha$ variando discretamente.

O exemplo do mapa logístico discutido mostra que a modelagem de sistemas dinâmicos pode ser usada para descrever o comportamento compelxo observado na natureza. O fato de podemos representar o comportamento dinâmico por meio de equações, faz com que modelos químicos, em especial eletroquímicos, se apresentem como protótipos interessantes no entendimento do comportamento complexo encontrado na natureza. Em eletroquímica as instabilidades temporais, são observadas em sistemas como eletrodeposição de metais, reações faradaica com inibição de filmes orgânicos e reações eletrocatalíticas, vem sendo corriqueiramente observadas e estudadas em diversos trabalhos na literatura [16-18].

\section{I.1.1 Instabilidades Cinéticas em Sistemas Eletroquímicos}

Uma grande quantidade de processos que ocorrem na interface eletrodo-solução pode, em princípio, apresentar comportamento instável numa certa faixa de parâmetros experimentais [19]. A cinética complexa em sistemas eletroquímicos está primeiramente associada à presença de auto-catálise no potencial da dupla camada, sendo essa instabilidade expressa na forma de uma resistência diferencial negativa (negative differential resistence, NDR) na curva estacionária de corrente-potencial [20,21]. Essa característica associada à presença de competição entre diferentes espécies por sítios ativos e a consequente depedência do recobrimento com o potencial relacionado pela isoterma de adsorção, fazem com que sistemas eletroquímicos apre- 
sentem comportamento complexo na forma de oscilações de corrente ou potencial [22-24].

Na eletro-oxidação de moléculas orgânicas pequenas, como por exemplo ácido fórmico, metanol, etanol e etileno glicol, postula-se que o mecanismo relacionado envolve duas vias reacionais [25]: (a) uma via direta, que regula a formação de intermediários ativos, esses uma vez formados reagem rapidamente levando a formação do produto final, que nesse caso é o $\mathrm{CO}_{2}$ e (b) uma via indireta, em que o $\mathrm{CO}$ adsorvido bloqueia a superfície sendo removido a altos potencias. Como um exemplo desse tipo de comportamento, temos a eletro-oxidação do metanol na superfície da platina policristalina em meio ácido, onde o $C O$ adsorvido começa a ser removido nos potenciais acima de $0.6 \mathrm{~V}$ por meio de reações com espécies oxigenadas como $\mathrm{OH}$. Geralmente, as instabilidades cinéticas observadas nestes sistemas são descritas em termos da classe dos osciladores eletroquímicos do tipo $H N-N D R$ [23], onde a nomenclatura refere-se à presença de uma $N D R$ em uma curva de polarização na forma de "N" parcialmente escondida (hidden, $H$ ) em condições voltamétricas.

Mecanisticamente, podemos representar o comportamento dinâmico por meio dos ciclos de retro-alimentação que relacionam a interação entre as isotermas de adsorção de espécies como $\mathrm{CO}_{a d}$ e $\mathrm{OH}_{a d}$, que atuam como veneno para a reação de fundo [26]. Especificamente, essas espécies "bloqueiam" em diferentes potenciais a eletro-oxidação direta de moléculas orgânicas e a interação associada à emergência de oscilações simples pode ser explicadas por meio de dois ciclos de retro-alimentação. O primeiro ciclo está relacionado com a retro-alimentação positiva que corresponde à etapa auto-catalítica e o segundo relacionado à retro-alimentação negativa referente à etapa de envenenamento da superfície por uma espécie bloqueante. A Figura 2 ilustra como esses ciclos de retro-alimentação podem atuar na explicação do comportamento oscilatório. 


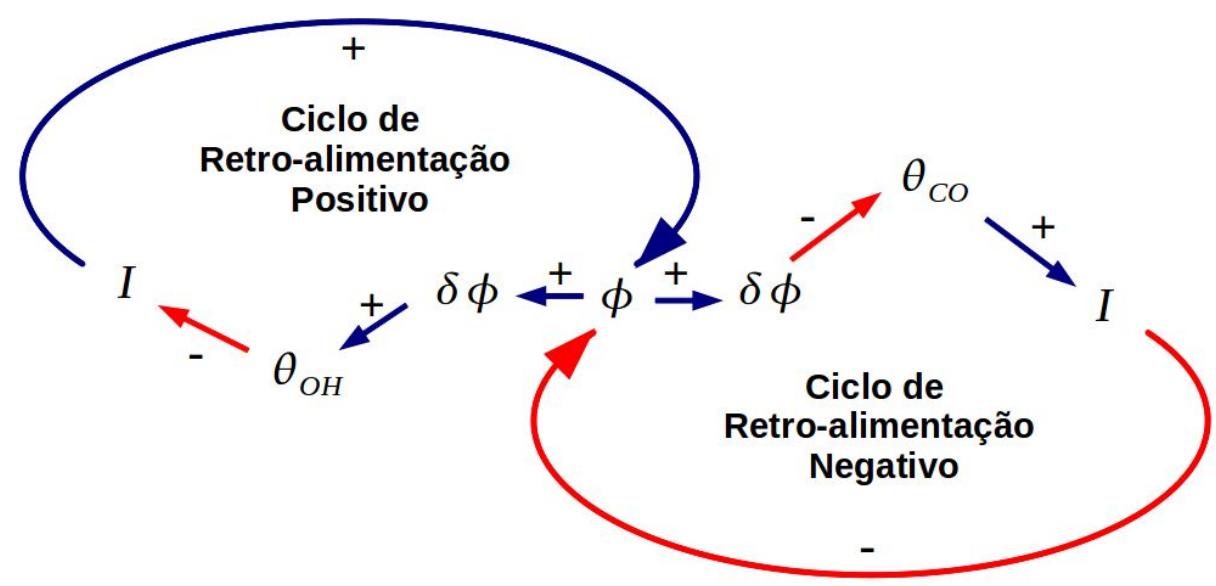

Figura 2: Esquema generalizado da interação entre os ciclos de retro-alimentação positivo e negativo.

Na Figura 2 é mostrado um esquema generalizado da interação entre dois ciclos de retro-alimentação, onde considera-se inicialmente que o sistema está sob controle potenciostático, ou seja, o potencial $U$ aplicado ao sistema é mantido constante. Como exemplo, assumese que o potencial do eletrodo, dado por $\phi$, sofre uma pequena variação $\delta_{+} \phi$ para valores um pouco mais positivos em torno de um valor fixo dado por $\phi_{f}$. Em resposta a essa perturbação a cobertura de $O H_{a d}$, dada por $\theta_{O H}$, aumenta, acarretando a diminuição da corrente total $I$. Essa variação leva o sistema a aumentar o potencial do eletrodo de forma auto-catalítica formando um ciclo de retro-alimentação positivo. Por outro lado, um aumento no recobrimento de $O H_{a d}$ promove uma diminuição no recobrimento de $\mathrm{CO}_{a d}$, a variável inibidora, que reage com $\mathrm{OH}_{a d}$ via o mecanismo de Langmuir-Hinshelwood promovendo assim o processo de reativação superficial e dessa forma disponibilizando novamente sítios ativos. Porém, uma diminuição da cobertura de $C O_{a d}$, representada por $\theta_{C O}$, causa um aumento na corrente total do sistema. Fazendo com que o potencial do eletrodo diminua, formando assim um ciclo de retro-alimentação negativo.

Uma importante característica dos osciladores do tipo $H N-N D R$ é que mecanisticamente eles possuem os pré-requisitos necessários para oscilarem tanto em modo galvanostático, ou corrente total mantida constante, quanto em modo potenciostático, i.e., potencial aplicado constante. Sob controle potenciostático, oscilações ocorrem a partir de certos valores de resistência conectada em série com o eletrodo de trabalho, de forma que a queda ôhmica permita certa liberdade ao potencial do eletrodo $\phi$, tendo em vista que o potencial aplicado $U$ está rela- 
cionado ao potencial do eletrodo através da equação: $U=\phi+I R_{t}$, em que $R_{t}$ representa a soma de todas as resistências em série do sistema. Além da importância na dinâmica temporal dos sistemas pontuais, a forma de controle da interface assume um papel fundamental na estruturação espaço-temporal.

O comportamento característico para experimentos com sistemas $H N-N D R$, pode ser visto em diversos trabalhos na literatura. Como exemplos podemos citar os trabalhos de Schell et al. [27, 28], referentes à oxidação eletroquímica de formaldeído na superfície de $P t$, assim como os artigos de Markovic e Ross [29] e Strasser et al. [30, 31] com relação à eletrooxidação do ácido fórmico.

\section{I.1.2 Protótipos da Dinâmica Temporal}

A modelagem da cinética complexa observada em sistemas químicos pode ser feita basicamente por dois caminhos diferentes. O primeiro tem como base uma descrição realista do mecanismo de reação das espécies envolvidas. Esse tipo de modelagem traz informações do comportamento químico, ou em nosso caso eletroquímico, do sistema estudado, como exemplos podemos citar os trabalhos de Eiswirth e colaboradores que tratam da modelagem mecanística do ácido fórmico e metanol [32,33]. Já o segundo baseia-se na hipótese global, levando em consideração as características comuns do comportamento observado em diversos sistemas. Apesar da modelagem global não trazer informações detalhadas do comportamento de um sistema específico, ela revela informações importantes do comportamento geral referente a uma classe de sistemas estudados, isso aplicado ao fato de que modelos genéricos são mais simples em relação aos modelos específicos, faz com que tais modelos sejam ferramentas importantes para o entendimento do comportamento observado em diferentes sistemas experimentais. Com base nisso, foi abordado nesse trabalho um modelo eletroquímico genérico proposto inicialmente por Krischer [19] que consiste de um sistema formado por três equações diferenciais ordinárias nãolineares, e acopladas. O sistema resltante foi obtido mediante a combinação de dois modelos simples formulado por Koper e Sluyters [34, 35] e descreve de maneira aproximada o comportamento de sistemas do tipo $N-N D R$ e $H N-N D R$. Para relatar como o modelo aqui estudado 
foi formulado, vamos definir primeiramente a classe do modelo $N-N D R$ formulado por Koper $[20,36]$ e logo em seguida definiremos a classe do modelo $H N-N D R$.

\section{I.1.2.1 Modelo N-NDR}

Foi comentado inicialmente que instabilidades cinéticas em sistemas eletroquímicos estão associadas ao fenômeno de auto-catálise no potencial da dupla camada, sendo expressas na forma de uma $N D R$. De fato, uma $N D R$ em uma curva na forma de $N$ forma-se muito facilmente e sua ocorrência é a razão pela quais oscilações ocorrem na maioria dos sistemas eletroquímicos. Para melhor entender a formação de uma $N D R$, vamos inicialmente considerar um circuito elétrico simples constituído por quatros elementos que representa, de forma equivalente, o comportamento de uma célula eletroquímica mantida sob controle potenciostático.

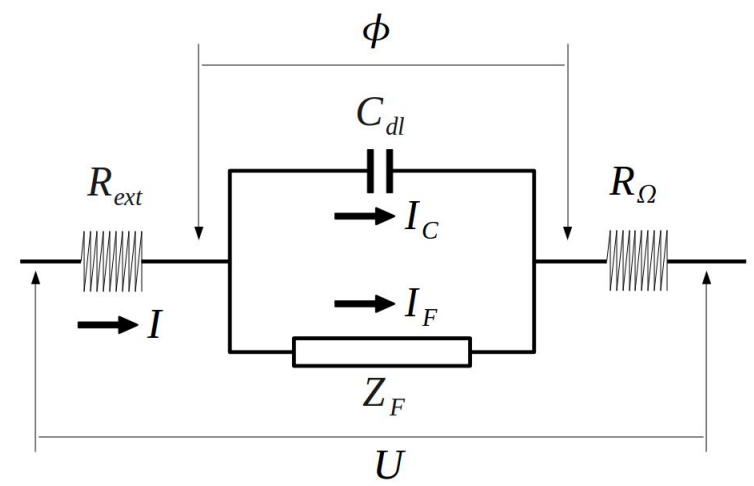

Figura 3: Circuito equivalente referente a uma interface eletrificada sob controle potenciostático.

Na Figura 3 temos que seus elementos são: $R_{\text {ext }}$ que corresponde à resistência externa conectada entre o eletrodo de trabalho e potenciostato, $R_{\Omega}$ a resistência da solução, $C_{d l}$ representa a capacitância da dupla camada elétrica, $\phi$ o potencial da dupla camada e $Z_{F}$ a impedância faradaica referente aos processos de transferências de carga na interface elétrica [37].

Aplicando a lei de Kirchoff ao circuito ilustrado na Figura 3, podemos relacionar o balanço de carga para obter uma equação que governa o comportamento dinâmico do potencial na dupla camada elétrica, essa relação é dada por: 


$$
I=I_{F}(\phi)+I_{C}
$$

sendo $I$ a corrente total do sistema, $I_{F}(\phi)$ a corrente faradaica ou reacional dada por $I_{F}=$ $n F A(\phi) c(\phi) k(\phi)$, em que $n$ é o número de elétrons envolvidos na reação, $F$ constante de Faraday, $c$ concentração de uma espécie e $k(\phi)$ que corresponde à constante cinética da reação. É importante relatar que a dependência de $A(\phi), c(\phi)$ e $k(\phi)$ com o potencial $\phi$ está relacionado com a adsorção de espécies na superfícies do eletrodo, assim como os fenômeno de transferência de elétrons envolvidos na dupla camada. Por fim, o último termo da Equação 2 dado por $I_{C}$ que corresponde à corrente capacitiva descrita pela Equação 3, em que $A$ é a área eletroativa do eletrodo.

$$
I_{C}=C_{d l} A \frac{d \phi}{d t}
$$

O potencial aplicado no sistema pode ser definido como $U=\left(R_{\text {ext }}+R_{\Omega}\right) I+\phi$, que substituindo na Equação 2 e juntamente com a Equação 3, fornece a Equação 4. Nessa equação, a resistência total é representada por $R_{t}=R_{e x t}+R_{\Omega}$. A Equação 4 é de fundamental importância para o entendimento das instabilidades cinéticas observadas em sistemas eletroquímicos.

$$
C_{d l} A \frac{d \phi}{d t}=-I_{F}(\phi)+\frac{U-\phi}{R_{t}}
$$

Para o entendimento do comportamento instável causado pela $N D R$, vamos primeiramente considerar a Equação 4 no estado estacionário ${ }^{\dagger}$ dado por:

$$
\begin{gathered}
\frac{d \phi_{e e}}{d t}=0 \\
I_{F}\left(\phi_{e e}\right)=\frac{U-\phi_{e e}}{R_{t}}
\end{gathered}
$$

\footnotetext{
$\dagger$ Uma vez que o comportamento dinâmico para um sistema é descrito por uma equação diferencial na forma de $\frac{d x}{d t}=f(x)$, o estado estacionário ou estado de equilíbrio representado pelo índice ee, acontece quando os pontos fixos satisfazem a condição de $f\left(x_{e e}\right)=0$, isso implica que a derivada da variável $x$ no tempo é zero ou seja $\frac{d x}{d t}=0$.
} 
A estabilidade no estado estacionário $\phi_{e e}$, pode ser avaliada pela aplicação de uma pequena perturbação $\delta \phi$ e observando a evolução dessa perturbação no tempo. Assim podemos escrever o potencial da dupla camada como $\phi=\phi_{e e}+\delta \phi$. Considerando agora a corrente faradaica como $I_{F}(\phi)=I_{F}\left(\phi_{e e}+\delta \phi\right)$, temos que ela pode ser escrita na forma:

$$
I_{F}\left(\phi_{e e}\right)=I_{F}\left(\phi_{e e}\right)+\left(\frac{d I_{F}}{d \phi}\right)_{\phi=\phi_{e e}} \delta \phi
$$

Ao observar o segundo termo da Equação 6, nota-se a definição de impedância faradaica, $Z_{F}$, dada por Koper [36], onde $Z_{F}^{-1}=\left(\frac{d I_{F}}{d \phi}\right)_{\phi=\phi_{e e}}$. Fazendo a substituição de todos esses termos na Equação 4, obtemos a Equação 7 que descreve o comportamento do sistema em função de uma pequena perturbação $\delta \phi$.

$$
C_{d l} A \frac{d \delta \phi}{d t}=-\left(\frac{1}{Z_{F}}+\frac{1}{R_{t}}\right) \delta \phi
$$

Analisando a Equação 7, percebe-se que as condições para o sistema se encontrar no estado estacionário estável é $Z_{F}>0$, uma vez que a resistência total é sempre positiva. Já para o estado estacionário instável temos que satisfazer duas condições simultâneas: (a) o modulo da impedância faradaica deve ser menor que a resistência total $\left|Z_{F}\right|<R_{t}$ e (b) a impedância faradaica deve ser negativa $Z_{F}<0$.

Mantendo a mesma linha de raciocínio só que agora no modo galvanostático, onde a corrente total $I$ é constante temos que a Equação 4 pode ser reescrita como:

$$
C_{d l} A \frac{d \phi}{d t}=-I_{F}(\phi)+I
$$

essa equação rege o comportamento do potencial da dupla camada em modo galvanostático.

Trabalhando com a Equação 8 sob a ação de uma pequena perturbação $\delta \phi$, concluimos que os estados estacionários instáveis são obtidos pela impedância faradaica negativa, $Z_{F}<0$. Essa observação relatada por Koper [36] deixa clara a importância da impedância faradaica negativa nos fenômenos oscilatórios observados tanto no modo potenciostático como galvanostático. Além da impedância, Koper relata também o papel da resistência externa para a formação 
de oscilações no modo potenciostático em um oscilador eletroquímico. A interpretação física para esses fenômenos observados é que sob condições potenciostáticas se faz necessário um elemento em série para permitir oscilações no potencial do eletrodo, de forma que, a queda de voltagem sobre o elemento em série irá oscilar exatamente fora de fase com o potencial do eletrodo [36].

Como discutido anteriormente, a principal causa das instabilidades em sistemas eletroquímicos é a presença de uma impedância faradaica negativa, $Z_{F}<0$, usualmente chamada de resistência diferencial negativa quando considera-se apenas a parte real. Um ponto muito importante a ser ressaltado aqui é a derivada da lei de Faraday em função do potencial $\phi$ mostrada na Equação 9, que fornece as condições para a obtenção de uma impedância negativa [36],

$$
\frac{d I_{F}}{d \phi}=Z_{F}^{-1}=n F\left[c(\phi) k(\phi) \frac{d A(\phi)}{d \phi}+A(\phi) k(\phi) \frac{d c(\phi)}{d \phi}+A(\phi) c(\phi) \frac{d k(\phi)}{d \phi}\right]
$$

Analisando esta expressão, nota-se que as causas para se ter uma impedância faradaica negativa são:

1. Uma variação negativa da área eletroativa em função do potencial, $\frac{d A(\phi)}{d \phi}<0$ causada pela adsorção de uma espécie inibidora como por exemplo reações de envenenamento causadas tanto pela dissolução de metal como adsorção de espécies [38-43].

2. A variação negativa da constante de velocidade, $\frac{d k(\phi)}{d \phi}<0$, pode ser causada pela adsorção de espécies que inibem a reação [44, 45].

3. A influência negativa da concentração, $\frac{d c(\phi)}{d \phi}<0$, pode ser atribuída aos fenômenos da dupla-camada elétrica, que em soluções diluídas tem a velocidade de transferência de elétrons afetada pelo potencial da dupla-camada, essa influência pode ser vista por exemplo nos trabalhos de Kariuki e Dewald [46, 47]. 


\section{I.1.2.1.1 Biestabilidade em Sistemas N-NDR}

O fenômeno não-linear mais simples encontrado, levando em consideração o estado estacionário para os sistemas $N-N D R$ é o comportamento biestável ou biestabilidade causada pela presença dos pontos de sela-nó na curva estacionária de corrente-potencial. Tal comportamento está associado à variável do sistema, nesse caso o potencial $\phi$, sendo encontrado tanto em controle galvanostático como potenciostático. O comportamento biestável para os sistemas $N-N D R$ com uma variável pode ser observado na Figura 4 que mostra um conjunto de gráficos calculados para Equação 4, como essa figura representa um esquema do comportamento dinâmico os índices numéricos foram omitidos. Já os valores as resistências $R_{t}, 5 R_{t}$ e $10 R_{t}$ referem-se aos valores para as resistências utilizadas na obtenção dos diagramas de bifurcação da Figura 4.

(a)

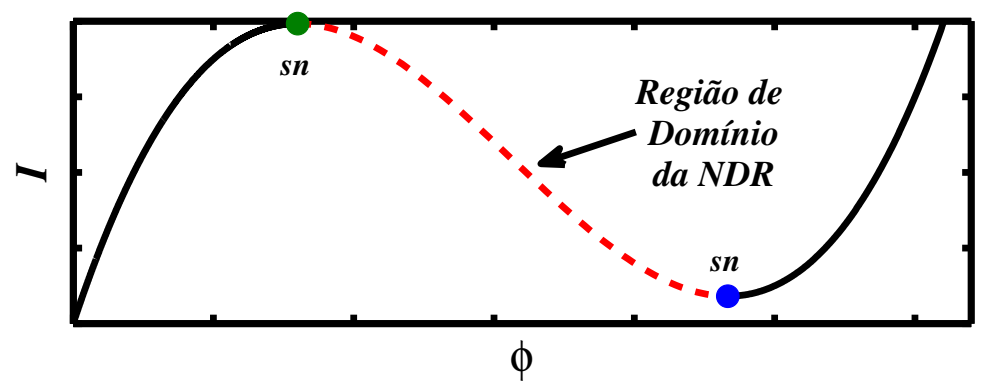

(b)

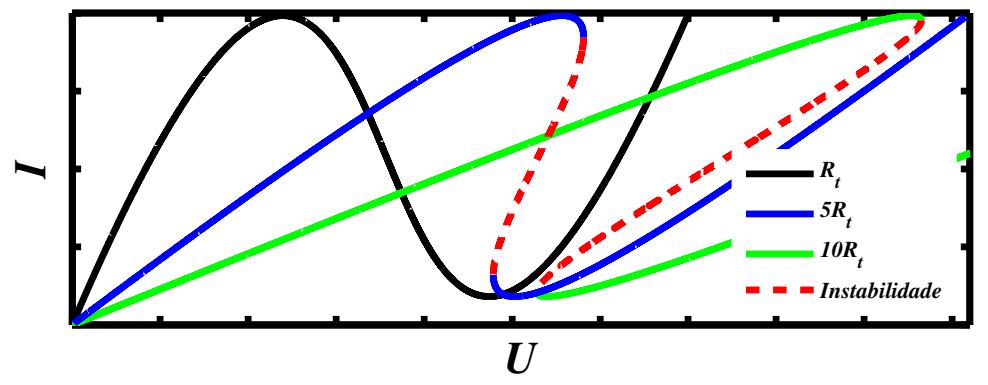

(c)

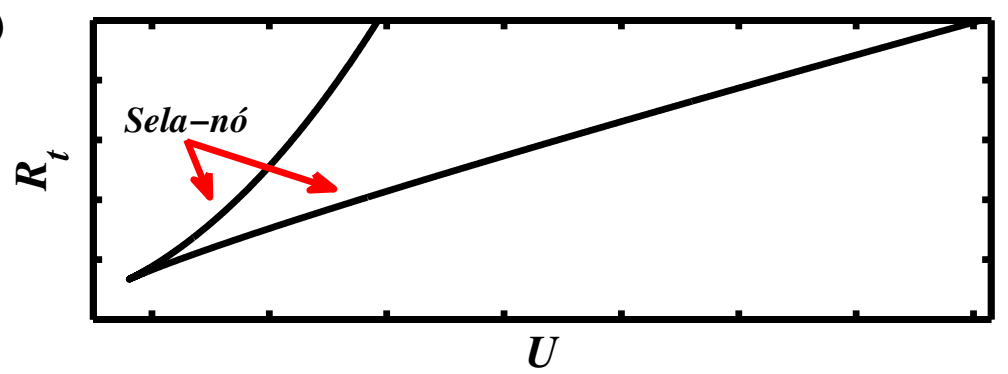

Figura 4: (a) curva no estado estacionário para $I$ versus $\phi$, (b) diagrama de bifurcação para $I$ versus o parâmetro $U$ e (c) diagrama de bifurcação sela-nó para o espaço de parâmetros $R_{t}$ versus $U$. 
Na Figura 4 (a) é exibido um gráfico de $I$ versus $\phi$ onde a região de domínio da NDR é expressa como uma linha tracejada na cor vermelha e os pontos verde e azul representam os pontos de sela-nó, representados por sn que correspondem às extremidades do domínio da NDR. O ramo dessa curva dá origem ao ciclo de retro-alimentação positivo e pode ser melhor explicado supondo uma pequena flutuação de $\phi$, localizada no ponto verde da Figura 4 (a), para valores maiores provocando uma diminuição de $I$ além de crescer auto-cataliticamente até o sistema atingir o outro ramo estável da curva $N$, representado pelo ponto azul, que possui uma inclinação positiva.

Se variamos o parâmetro $U$ na Equação 4, o comportamento dinâmico do sistema será alterado, resultando em uma bifurcação de sela-nó. Tal variação faz com que pontos fixos sejam criados ou destruídos acarretando na mudança de estabilidade do sistema. Isso faz com que dois estados estacionários definidos como estável e instável se formem. Esse comportamento pode ser observado na Figura 4 (b), que mostra exatamente a resposta numa curva $I$ versus $U$ para uma variação da resistência $R_{t}$. Note que para certo valor de $R_{t}$ a curva estacionária indicada pela linha preta na Figura 4 (b), não apresenta região de instabilidade formada pela presença dos pontos de sela-nó. Nesse caso, todos os ramos da curva podem ser acessados pelo sistema. Entretanto, quando aumentamos o valor de $R_{t}$ para $5 R_{t}$ e $10 R_{t}$ em relação ao valor inicial, curvas azul e verde na Figura 4 (b) respectivamente, percebemos a formação de regiões instáveis, linhas vermelhas na Figura 4 (b), delimitadas pelos pontos de sela. Tais regiões de instabilidade não são acessadas pelo sistema, que chegando nessas regiões salta para o outro ramo estável da curva. Essa característica é muito interessante pois provoca no sistema um comportamento chamado de histerese, que esta relacionado com diferentes estados no sistema.

Outro ponto muito importante a ser ressaltado nos diagramas de bifurcações da Figura 4 (b), é a inclinação das curvas quando $R_{t}$ aumenta. Observa-se que na medida em que $R_{t}$ aumenta vai ficando cada vez mais difícil acessar a região de instabilidade no sistema. Quando o valor de $R_{t}$ é muito alto o sistema passa a não enxergar a instabilidade da curva, que nessas condições não deve apresentar biestabilidade. Tal comportamento é compatível com a condição galvanostática obtida para o limite de $R_{t} \rightarrow \infty$ e $U \rightarrow \infty$ na Equação 10 para a corrente total do sistema, em que o ramo instável nunca será atingido pelo sistema. Essa característica é 
muito interessante, pois implica em dizer que para sistemas onde o comportamento apresentado é $N-N D R$, jamais poderão apresentar oscilações em condições galvanostática.

$$
I=\lim _{\substack{R_{t} \rightarrow \infty \\ U \rightarrow \infty}} \frac{U-\phi}{R_{t}}
$$

Analisando a Figura 4 (c), observa-se o comportamento da bifurcação de sela-nó para o espaço de parâmetros $R_{t}$ versus $U$. Como mostrado no diagrama de bifurcação, Figura 4 (c), a região de biestabilidade localizada no interior da curva de sela aumenta na medida em que $R_{t}$ e $U$ aumenta. É interessante notar que o aumento na região de biestabilidade colabora para que o sistema não tenha acesso ao ramo instável, dessa forma confirmando o resultado obtido para a Figura 4 (b).

\section{I.1.2.1.2 Oscilações em Sistemas N-NDR}

Para observar comportamento oscilatório em modelos $N-N D R$ um segundo processo deve ser introduzido. Esse processo corresponde ao ciclo de retro-alimentação negativo, como descrito na Figura 2. O ciclo de retro-alimentação pode ser explicado por uma função que relaciona a dependência da concentração com o tempo. Koper e Sluyters [20] elaboraram um modelo matemático simplificado que descreve o comportamento oscilatório para sistemas $\mathrm{N}-$ $N D R$. Koper e Sluyters propõem que a dependência da concentração superficial $c$ com o tempo é dada em termos do valor no seio da solução $c^{b}$, assumindo a simplificação de que o gradiente de concentração próximo ao eletrodo é sempre linear e consequentemente a camada de difusão tem sempre a mesma espessura $\delta$. Um esquema ilustrativo da variação no perfil de concentração pode ser visualizado na Figura 5. 


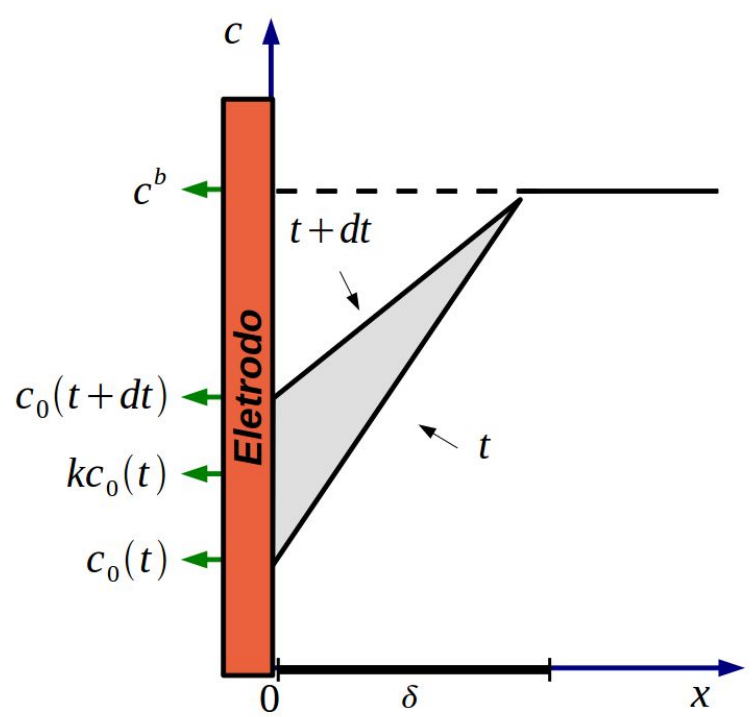

Figura 5: Esquema ilustrativo da variação de concentração próximo ao eletrodo no intervalo de $d t$.

A área cinza mostrada na Figura 5 pode ser comparada à diferença entre a quantidade de matéria que difunde para a superfície do eletrodo e a quantidade que reage na superfície. Essa diferença pode ser expressa como:

$$
\frac{1}{2}\left[c_{0}(t+d t)-c_{0}(t)\right] \delta=\frac{D\left(c^{b}-c_{0}\right)}{\delta} d t-k(\phi) c_{0}(t) d t
$$

em que $D$ é o coeficiente de difusão, $\delta$ refere-se à espessura da dupla camada, $c_{0}$ a concentração próxima à superfície do eletrodo, $c^{b}$ a concentração no volume do eletrólito e $k(\phi)$ a constante cinética da reação. Essa equação é equivalente à Equação 12, que descreve o comportamento da concentração de uma espécie variando no tempo em função do potencial na dupla camada.

$$
\frac{d c}{d t}=-\frac{2}{\delta} c k(\phi)+\frac{2 D}{\delta^{2}}\left(c^{b}-c\right)
$$

Quando adicionamos a Equação 4 juntamente com a Equação 12, formamos um modelo que mimetiza o comportamento oscilatório de forma simplificada para um sistema $N-N D R$.

O modelo pode ser simplificado fazendo uma adimensionalização das equações por meio das seguintes relações: 


$$
\begin{gathered}
\frac{n F}{R T} \phi \rightarrow \phi, \quad \frac{n F}{R T} U \rightarrow U, \quad \frac{c}{c^{b}} \rightarrow c, \quad k(\phi) \frac{\delta}{D} \rightarrow k(\phi), \quad \frac{2 D}{\delta^{2}} t \rightarrow t \\
\varepsilon^{\prime}=\frac{2 C_{d l} R T}{c^{b} n^{2} F^{2} \delta} \text { e } \rho^{\prime}=\frac{c^{b} D n^{2} F^{2} R_{t} A}{\delta R T}
\end{gathered}
$$

A versão adimensional do modelo gerada pelas relações descritas fornece um conjunto de Equações 13 e 14.

$$
\begin{aligned}
\varepsilon^{\prime} \frac{d \phi}{d t} & =-c k(\phi)+\frac{U-\phi}{\rho^{\prime}} \\
\frac{d c}{d t} & =-c k(\phi)+1-c
\end{aligned}
$$

No modelo adimensional os símbolos $\varepsilon^{\prime}$ e $\rho^{\prime}$ representam os parâmetros de controle da escala de tempo do potencial e a resistência adimensional respectivamente. Uma visão geral do comportamento dinâmico para o modelo $N-N D R$ simplificado, proposto por Koper e Sluyters, pode ser descrito por meio das Figuras 6 (a) e (b).
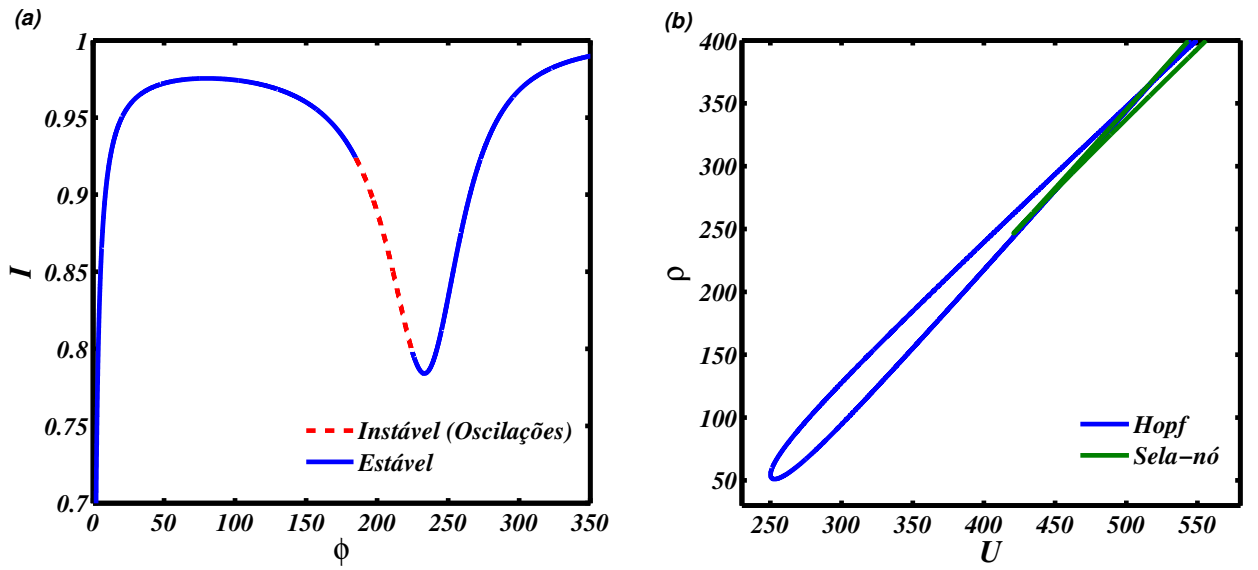

Figura 6: (a) curva no estado estacionário para I versus $\phi$ e (b) diagrama de bifurcação sela-nó para o espaço de parâmetros $\rho^{\prime}$ versus $U$.

A Figura 6 (a) mostra uma curva estacionária no plano de fase dado por $I$ versus $\phi$ para o modelo $N-N D R$ formado pelo conjunto de equações diferenciais em 13 e 14 . Observe que a região da curva correspondente à linha vermelha tracejada refere-se à região de instabilidade onde são observadas oscilações simples. Já a Figura 6 (b) mostra um típico diagrama 
de parâmetros, em que é possível observar as bifurcações de Hopf e sela-nó para esse modelo, linhas azul e verde na Figura 6 (b), respectivamente. A bifurcação de Hopf mostra a região de parâmetros em que são observadas oscilações de corrente no tempo. Note que essa região é fechada indicando que para valores de $\rho^{\prime}$ e $U$ tendendo ao infinito o sistema não apresenta oscilações.

\section{I.1.2.2 Modelo HN-NDR}

Foi comentada anteriormente a existência de duas classes de sistemas que apresentam comportamento dinâmico relacionado a uma curva no formato de $N$ em condições de correntepotencial: $N-N D R$ e $H N-N D R$. Nesse trecho da Tese será discutido um pouco do modelo $H N-N D R$ e logo em seguida será apresentado um protótipo sugerido inicialmente por Krischer [19]. A dinâmica apresentada por esse protótipo constituído por três equações diferenciais será o assunto central abordado na Parte I dessa Tese.

\section{I.1.2.2.1 Comportamento Oscilatório para o Modelo HN-NDR Simples}

A forma mecanística mais comum pela qual uma $N-N D R$ possa ser ocultada é fazendo a consideração de que uma espécie inibidora do processo de transferência de elétrons que se adsorve na superfície do eletrodo. Tais espécies podem estar dissolvidas no eletrólito ou podem ser formadas durante a aplicação de um potencial. Esse tipo de comportamento pode ser observado em quase todos os experimentos envolvendo a eletro-oxidação de moléculas orgânicas pequenas.

Koper e Sluyters [35] propuseram um modelo simplificado constituído por duas equações diferenciais ordinárias, onde uma delas descreve o comportamento do recobrimento de uma espécie na superfície do eletrodo. Foi ainda discutido que as três explicações mais frequentes para os fenômenos oscilatórios citados na literatura [48] levam em consideração: (a) os desvios das leis de adsorção de Langmir, (b) as reações autocatalíticas na superfície do eletrodo gerando sítios livres e (c) transformações estruturais impulsionadas pelo adsorbato. Levando isso em 
consideração, Krischer [19, 49] propôs um modelo simplificado formado por duas equações diferenciais. Esse modelo também pode ser visto como uma derivação do modelo de Koper e Sluyters.

O modelo de Krischer considera que uma espécie eletroativa sofre uma oxidação ou uma redução formando uma mova espécie $P$ com caráter inibidor, que tem sua isoterma de adsorção dependente do potencial aplicado. A formulação matemática para esse modelo é expressa pelo conjunto de Equações 15 e 16.

$$
\begin{gathered}
C_{d l} \frac{d \phi}{d t}=-n F c k_{E}(\phi)(1-\theta)+\frac{U-\phi}{A R_{t}} \\
\frac{d \theta}{d t}=k_{p}\left[\theta_{0}(\phi)-\theta\right]
\end{gathered}
$$

onde $\theta$ representa o recobrimento da espécie $P, \theta_{0}(\phi)$ é uma função que descreve o comportamento do recobrimento no estado de equilíbrio e $k_{p}$ corresponde a uma constante que determina a velocidade de relaxação do recobrimento em relação ao equilíbrio.

O conjunto formado pelas Equações 15 e 16 pode ser adimensionalizado usando as relações abaixo:

$$
\begin{gathered}
\frac{n F}{R T} \phi \rightarrow \phi, \quad \frac{n F}{R T} U \rightarrow U, \quad k_{E}(\phi) \rightarrow k_{0} k(\phi), \quad k_{p} t \rightarrow t \\
\varepsilon^{\prime \prime}=\frac{C_{d l} R T k_{p}}{n^{2} F^{2} k_{0}} \text { e } \rho^{\prime \prime}=\frac{n^{2} F^{2} R_{t} A k_{0}}{R T}
\end{gathered}
$$

Essas relações geram o sistema adimensional dado pelas Equações 17 e 18. Em que os parâmetros $\varepsilon^{\prime \prime}$ e $\rho^{\prime \prime}$ se referem à escala de tempo do potencial e à resistência adimensional para o modelo $H N-N D R$.

$$
\begin{gathered}
\varepsilon^{\prime \prime} \frac{d \phi}{d t}=-k(\phi)(1-\theta)+\frac{U-\phi}{\rho^{\prime \prime}} \\
\frac{d \theta}{d t}=\theta_{0}(\phi)-\theta
\end{gathered}
$$




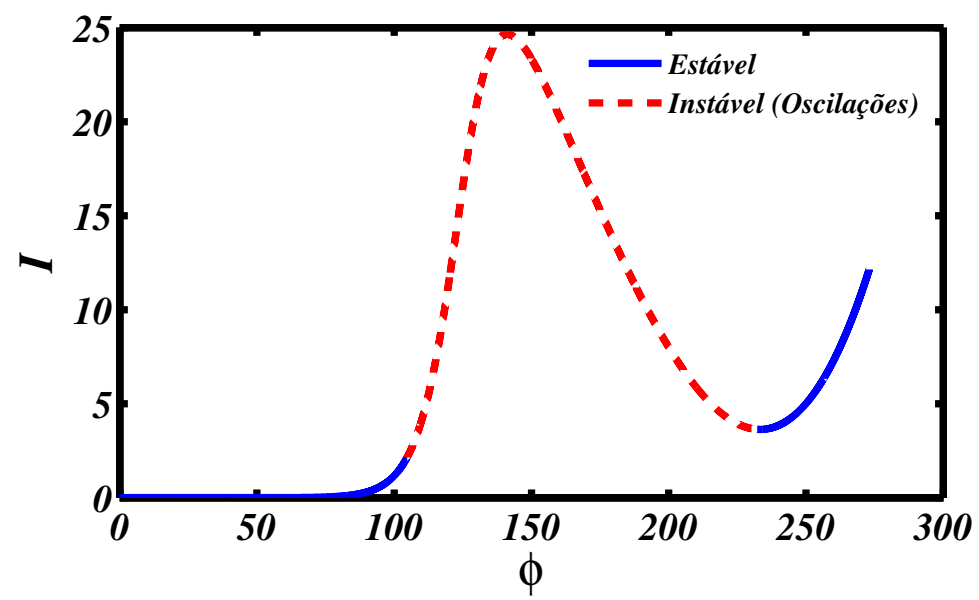

Figura 7: Curva estacionária para o modelo $H N-N D R$ no espaço de fase $I$ versus $\phi$. A linha vermelha tracejada corresponde à região instável do modelo.

A Figura 7 mostra uma curva no estado estacionário levando em consideração a presença de uma espécie química que bloqueia a superfície do eletrodo. Observe que agora temos uma $N-N D R$ parcialmente escondida, onde a linha vermelha tracejada corresponde à região de instabilidade da $H N-N D R$. Esta mesma curva, assim como a curva da $N-N D R$, tem como característica mudar sua inclinação quando aumentamos a resistência externa. Entretanto, ao contrário da curva $N-N D R$, a curva $H N-N D R$ sempre terá sua região de instabilidade acessada mesmo se a curva estiver inclinada devido ao valor elevado do parâmetro $\rho$ ". Tal comportamento faz com que em modo galvanostático o sistema apresente oscilações.

\section{I.1.2.2.2 Modelo HN-NDR Complexo}

O modelo completo consiste de três equações diferenciais ordinárias e descreve a evolução temporal do potencial do eletrodo, da concentração superficial de uma espécie eletroativa à superfície eletródica e da cobertura de uma espécie bloqueante, resultando da combinação dos subsistemas $N-N D R$ e $H N-N D R$, referentes aos modelos descritos pelas Equações 4, 12 , 15 e 16 respectivamente. 


$$
\begin{gathered}
C_{d l} \frac{d \phi}{d t}=-n F c k(\phi)(1-\theta)+\frac{U-\phi}{A R_{t}} \\
\frac{d c}{d t}=-\frac{2}{\delta} c k(\phi)+\frac{2 D}{\delta^{2}}\left(c^{b}-c\right) \\
\frac{d \theta}{d t}=k_{p}\left[\theta_{0}(\phi)-\theta\right]
\end{gathered}
$$

As equações adimensionais podem ser obtidas usando as relações matemáticas logo abaixo, onde temos que o parâmetro de controle da escala de tempo da variação da concentração é representado por $\mu, \varepsilon$ refere-se ao parâmetros de controle da escala de tempo do potencial, $\rho$ a resistência adimensional e $U$ o potencial aplicado ao sistema. Os outros parâmetros correspondem aos mesmos citados anteriormente.

$$
\begin{aligned}
\frac{n F}{R T} \phi & \rightarrow \phi, \quad \frac{n F}{R T} U \rightarrow U, \quad \frac{c}{c^{b}} \rightarrow c, \quad \frac{\delta}{D} k(\phi) \rightarrow k(\phi), \quad k_{p} t \rightarrow t \\
\varepsilon & =k_{p} \frac{C_{d l} R T \delta}{c^{b} n^{2} F^{2} D}, \quad \mu=k_{p} \frac{\delta^{2}}{2 D} \quad e \rho=R_{t} \frac{A c^{b} D n^{2} F^{2}}{\delta R T}
\end{aligned}
$$

O modelo adimensional é fornecido pelo conjunto de Equações 22, 23 e 24.

$$
\begin{gathered}
\varepsilon \frac{d \phi}{d t}=-c(1-\theta) k(\phi)+\frac{U-\phi}{\rho} \\
\mu \frac{d c}{d t}=-c(1-\theta) k(\phi)-c+1 \\
\frac{d \theta}{d t}=\theta_{0}(\phi)-\theta
\end{gathered}
$$

No caso galvanostático, a Equação 22 é modificada pela substituição do segundo termo da equação que corresponde à lei de Ohm pela corrente $I$. As funções para as constantes de velocidade e recobrimento em função do potencial dadas por: 


$$
\begin{gathered}
k(\phi)=0,00002 \phi^{3}-0,0094 \phi^{2}+1,12 \phi \\
\theta_{0}(\phi)=\left[1+\exp \left(\frac{\phi-\phi_{0}}{b}\right)\right]^{-1}
\end{gathered}
$$

onde os valores dos termos referentes à Equação 26 são: $\phi_{0}=124,6$ e $b=7,1204$.

Um fator importante a ser ressaltado sobre a constante de recobrimento dada pela Equação 26 é o seu significado físico, que segundo Koper [20] deve relacionar o comportamento de $\theta$ causado pelos processos de envenenamento na superfície do eletrodo. 


\section{I.2 Objetivos}

Nessa primeira parte da Tese será apresentado um estudo criterioso do comportamento dinâmico apresentado pelo modelo genérico $H N-N D R$. Tal modelo é constituído por três equações diferenciais não-lineares acopladas, assumindo como variáveis do sistema a adsorção de uma espécie que bloqueia a superfície do eletrodo $\theta$, a concentração do reagente no plano interfacial $c$ e o potencial interfacial $\phi$. A principal característica apresentada por esse modelo é a descrição do comportamento complexo observado na eletro-oxidação de várias moléculas orgânicas pequenas. Tal estudo é de fundamental importância para compreender, do ponto de vista dinâmico, os resultados experimentais. Outro ponto abordado nessa parte da Tese consiste numa discussão com relação à formação das estruturas observadas no interior das bifurcações de Hopf por meio do comportamento homoclínico de sela-nó e sela-foco apresentado para esse modelo.

Para sustentar nossa argumentação o problema foi abordado utilizando técnicas numéricas de continuação e integração para construir os diagramas de bifurcações e diagramas bidimensionais de estabilidade, de Lyapunov e de período. 


\section{I.3 Descrição das Simulações}

Uma dificuldade quando lidamos com equações diferenciais não-lineares é que a resolução analítica não é factível. Tal característica nos leva a interpretar um conjunto de equações como um campo vetorial que pode ser feita analiticamente para um conjunto simplificado. Contudo, quando temos um conjunto de equações não-lineares mais complexo se faz necessário o uso de ferramentas numéricas [50].

Os métodos numéricos empregados para se trabalhar com sistemas de equações diferenciais são conhecidos com métodos de continuação numérica [51]. Esses métodos são baseados na localização e continuação de curvas de bifurcação no espaço de parâmetros e variáveis. A utilização de tais ferramentas agrega algumas vantagens como, por exemplo, (a) localização das regiões instáveis e construção de suas respectivas bifurcações e (b) identificação dos diversos tipos de instabilidade existentes referente aos pontos fixos no sistema estudado. Entretanto, tais métodos têm a limitação de não poderem identificar regiões caóticas, assim como regiões periódicas dentro de regiões caóticas, o que torna essa metodologia insuficiente para descrever o comportamento dinâmico no interior da bifurcação.

Outra limitação, agora com relação aos pacotes de softwares disponíveis para fazer o tratamentos dos sistemas tais como AUTO [52] e MATCONT [53], é o fato de não ser possível construir curvas de superfície com regiões de estabilidade, assim como descrever o tipo de estabilidade existente na superfície. Problema esse, que é facilmente resolvido utilizando métodos que resolvem sistemas não-lineares como, por exemplo, o método de Newton [54] e o método das nullclines [13].

A seguir serão descritos em detalhes as metodologias utilizadas para calcular os diagramas de estabilidade, de Lyapunov e de período. 


\section{I.3.1 Diagramas de Bifurcação}

Os diagramas de bifurcação mostrados nessa Tese, referente às linhas de bifurcação apresentadas, foram calculados usando o software MATCONT. No cálculo foi utilizado um passo variado de 0,01 como valor máximo e $1 \times 10^{-15}$ como mínimo. O motivo de escolher essa região de variação se deve à necessidade de tentar localizar outros tipos de pontos fixos no interior da bifurcação, porém mesmo com um passo tão pequeno não foi possível descrever as regiões com estruturas finas no interior da bifurcação devido ao fenômeno de multiesbilidade existente no modelo.

Já os gráficos de estabilidade foram calculados usando o método das nullclines ao invés do método de Newton. O motivo de não utilizar o método de Newton, se deve à extrema dificuldade na convergência dentro de uma região delimitada por pontos de sela-nó. Essa dificuldade se deve ao fato da matriz Jacobiana possuir pontos de singularidades nessa região, que tornam a solução do sistema não-linear impossível.

O método das nullclines é baseado na análise dos limites para os quais uma função $f(x)$ se aproxima de zero, onde essa função é escrita substituindo as variáveis diferentes de $x$ pelas suas respectivas nullclines.

Como demonstração vamos calcular a função $f(\phi)$ para o modelo constituído das Equações 22, 23 e 24, em que a constante de velocidade $k(\phi)$ é expressa na Equação 25 e a constante de recobrimento $\theta_{0}(\phi)$ é dada pela Equação 26 .

As nullclines para o modelo $H N-N D R$ adotado são representadas por $\theta_{n}$ e $c_{n}$, Equações 27 e 28 repctivamente. Já a $f(\phi)$ é dado pela Equação 29.

$$
\begin{gathered}
\theta_{n}=\left[1+\exp \left(\frac{\phi-\phi_{0}}{b}\right)\right]^{-1} \\
c_{n}=\frac{1}{1+k(\phi)\left(1-\theta_{n}\right)} \\
f(\phi)=-c_{n} k(\phi)\left(1-\theta_{n}\right)+\frac{\phi-U}{\rho}
\end{gathered}
$$


Conhecendo a função $f(\phi)$, são calculados os limites onde a função é zero dentro de um critério pré-estabelecido. O critério assumido para considerar o zero da função foi estimar os pontos entre os limites $\pm 1 \times 10^{-4}$. Após a obtenção dos zeros da função foi realizado um ajuste spline cúbico para igualar os vetores formando seis matrizes, As três primeiras são referentes às variáveis do sistema no estado estacionário $\left(\phi_{e e}, c_{e e}\right.$ e $\left.\theta_{e e}\right)$, duas são os parâmetros $U$ e $\rho$ corrigidos e a última corresponde à matriz de estabilidade dada por $\mathbf{A}$. Na matriz de estabilidade A estão contidas as informações com respeito ao tipo de estabilidade assumida pelos pontos fixos no estado estacionário.

\section{I.3.2 Diagramas de Lyapunov}

Como discutido anteriormente os métodos de continuação disponíveis nos softwares são limitados e não oferecem condições de determinar o comportamento no interior das bifurcações em um dado sistema. Para resolver tal problema foram calculados gráficos de parâmetros onde foi feita uma estimativa numérica por meio dos expoentes de Lyapunov.

Para estimar os expoentes de Lyapunov foi utilizado o algoritmo proposto por Wolf et al. [55], onde o sistema de equações é integrado juntamente com o sistema obtido após a linearização. A integração do sistema não-linear se faz necessária nesse caso pelo simples motivo de não poder se determinar uma solução analítica para o sistema diferencial.

O método de integração utilizado para resolver os sistemas de equações diferenciais foi o de Rung-Kutta padrão de quarta ordem com passo fixo de 0,01 .

No cálculo do expoente de Lyapunov foram adotados os seguintes procedimentos:

1. Integra-se o sistema nas condições iniciais de $\phi=1, c=0.1$ e $\theta=0.1$ dentro de uma faixa de tempo considerada entre $0<t<2000$. Esse procedimento tem por finalidade eliminar a existência do transiente com o propósito de melhorar a convergência.

2. Após essa primeira integração são coletadas as novas condições iniciais das variáveis referentes ao sistema no ponto $t=2000$. Feito isso, é reiniciado o cálculo com as novas 
condições iniciais para o intervalo de tempo entre $0<t<1000$. Esse intervalo foi selecionado por apresentar o melhor custo/benefício entre o tempo gasto para integração e a convegência dos expoentes. A utilização de um tempo maior que 1000 não altera de maneira significativa os resultados.

3. Tendo coletado os expoentes de Lyapunov referente ao ponto $t=1000$ na série temporal, salva-se um conjunto formado por três matrizes de ordem $N x M$, onde $N$ e $M$ fazem referência às variações aplicadas aos parâmetros analisados. Cada matriz representa um expoente em uma direção diferente do espaço de fase.

Após efetuar essas três etapas, são somadas as matrizes que representam os expoentes de Lyapunov $\lambda_{1}$ e $\lambda_{2}$ para introduzir as informações com respeito ao comportamento periódico do sistema, uma vez que na condição caótica o valor do segundo expoente de Lyapunov é praticamente zero. A Tabela 1 mostra um resumo do comportamento dinâmico esperado quando se analisa os expoentes de Lyapunov para um espaço de fase tridimensional.

Tabela 1: Relação dos comportamentos dinâmicos esperados em função dos sinais e intensidades dos expoentes de Lyapunov.

\begin{tabular}{c|c|c|c} 
Comportamento dinâmico & $\lambda_{1}$ & $\lambda_{2}$ & $\lambda_{3}$ \\
\hline Pontos fixos estáveis & - & - & - \\
\hline Caótico & + & 0 & - \\
\hline Periódico & 0 & - & - \\
\hline Quasiperiódico & 0 & 0 & - \\
\hline
\end{tabular}

Terminada a obtenção da matriz dos expoentes de Lyapunov $\left(\lambda_{1}+\lambda_{2}\right)$ é feita uma normalização, onde todos os expoentes positivos são divididos pelo maior expoente e todos os negativos pelo menor expoente. Essa normalização serve para definir melhor as regiões no diagrama de Lyapunov e distribuir de forma equivalente as escalas de cores para o mapa de cor. 


\section{I.3.3 Diagramas de Período}

Apesar dos diagramas de Lyapunov descrevem o comportamento dinâmico no interior das bifurcações, não é possível caracterizar as regiões periódicas do comportamento dinâmico. Contudo, tal limitação pode ser complementada por meio da construção de diagramas de período.

Os diagramas de período abordados nesse trabalho foram calculados usando um algoritmo proposto pelos próprios autores, sendo que uma parte desse algoritmo escrito em pseudocódigo [56], onde é feita a estimativa do período de uma série temporal, é descrita na Tabela

2.

Tabela 2: Método proposto escrito em pseudocódigo para estimar os períodos no interior de uma bifurcação.

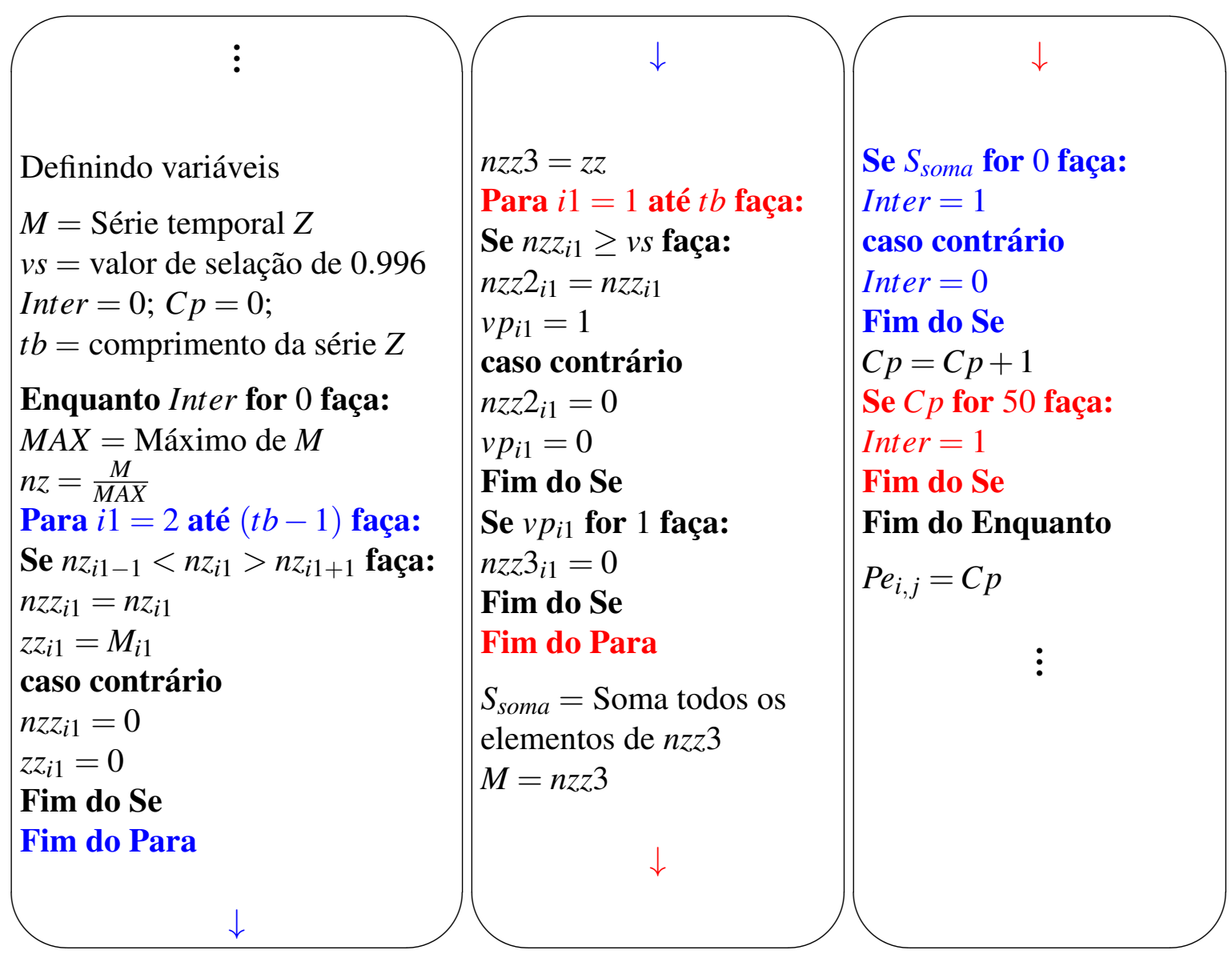


Esse algoritmo propõe estimar os períodos das séries temporais por meio de duas etapas simples que são:

1. Encontrar o máximo da intensidade da série temporal para fazer uma normalização de forma a poder comparar as séries com um mesmo processo de descriminação de intensidades referentes aos picos de oscilações. Além disso, a normalização também atuará como um agente para intensificar as diferenças entre os picos que serão comparados pelo valor de seleção dado por $v s=0,996$. Esse valor diz que o programa irá considerar como iguais os picos que tiverem uma diferença de $4 \times 10^{-3}$ no valor da intensidade normalizada.

2. Tendo identificado os picos da série temporal, o programa irá retirar esses picos que possuem a mesma intensidade normalizada. Essa etapa irá se repetir até que todos os picos existentes na série sejam retirados e o número de vezes em que essa etapa é repetida é proporcional ao período morfológicos da série temporal representado por Pe no Algoritmo descrito na Tabela 2.

O procedimento utilizado no cálculo dos diagramas de período é praticamente o mesmo dos diagramas de Lyapunov, com duas únicas exceções. Uma com relação ao segundo tempo de integração do sistema que foi de $0<t<500$ ao invés de $0<t<1000$ como no diagrama de Lyapunov e a outra exceção é com relação ao passo utilizdo para realizar a integração do sistema pela segunda vez com as novas condições iniciais que foi de 0,001 . Esse passo foi selecionado para minimizar as diferenças nas intensidades normalizadas durante a etapa de seleção dos picos na série temporal. 


\section{I.4 Resultados e Discussão}

\section{I.4.1 Análise do Modelo Temporal}

\section{I.4.1.1 Discussão Geral do Modelo}

Alguns resultados para o modelo híbrido constituído pelas equações diferenciais 22, 23 e 24 são mostrados na Figura 8 que descreve os perfis estacionários de $k(\phi)$ e $\theta_{0}(\phi)$ em função de $\phi$. Os comportamentos dos perfis estacionários mostram as principais características do modelo em termos da curva $N D R$ presente na forma de $N$ (linha preta), a isoterma de adsorção referente à espécie que envenena a superfície do eletrodo (linha vermelha) e a combinação da curva $N$ com a isoterma de adsorção representando o comportamento da corrente faradaica (linha azul).

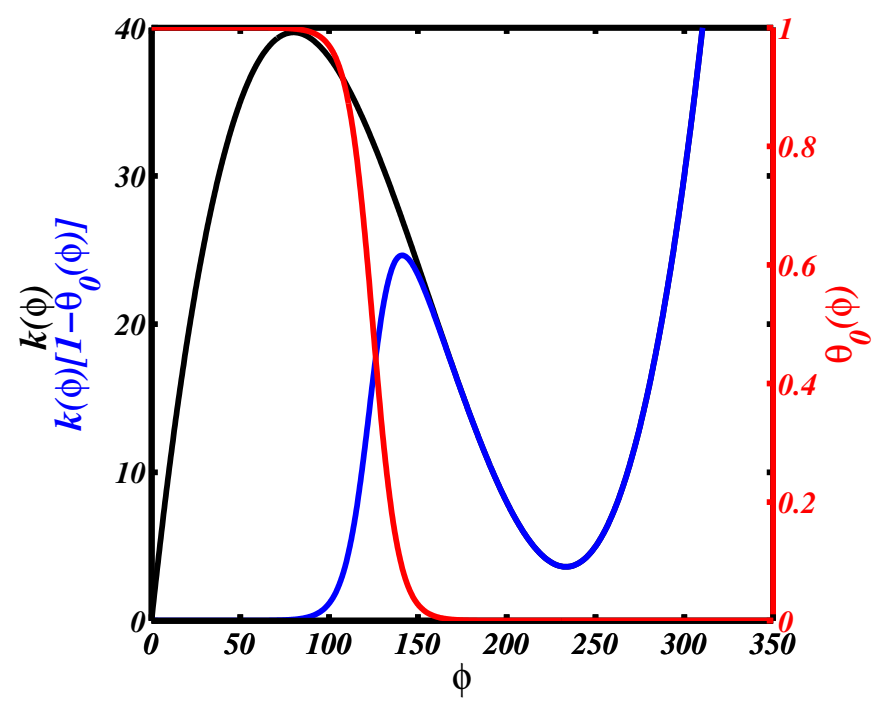

Figura 8: Perfis estacionários das contribuições para o modelo constituído pelas Equações 19, 20 e 21 , onde a linha preta corresponde ao perfil $N-N D R$, linha vermelha ao recobrimento da espécie bloqueante e a linha azul o comportamento observado $H N-N D R$. 
Como aparente na Figura 8, a resistência diferencial negativa da curva $N$ original é parcialmente escondida pela adsorção de uma espécie eletroativa. O comportamento da corrente faradaica, representado pela curva azul na Figura 8, é totalmente comparável ao observado em inúmeros sistemas experimentais tais como a eletro-oxidação de ácido fórmico [57-61], metanol $[62,63]$, etanol $[64,65]$ e formaldeído $[66,67]$ sobre platina. Como nos casos dos exemplos experimentais nesta classe, o modelo genérico do oscilador eletroquímico $H N-N D R$ [19] discutido aqui suporta oscilações de corrente para uma combinação de parâmetros entre $U$, $\rho, \varepsilon$ e $\mu$. As Figuras 9 (a) e (c) ilustram a resposta oscilatória da corrente faradaica para os valores de $\varepsilon=0,001, \mu=50$ e uma determinada resistência global dada por $\rho=106,7$ com dois valores diferentes de potencial aplicado, em que o valor de $U=244$, Figura 9 (a), apresenta o comportamento periódico. Já a Figura 9 (c) obtida para $U=246$, demonstra o comportamento caótico. As Figuras 9 (b) e (d) fazem referência aos atratores periódico e caótico no espaço de fase $\phi$ versus $\theta$.
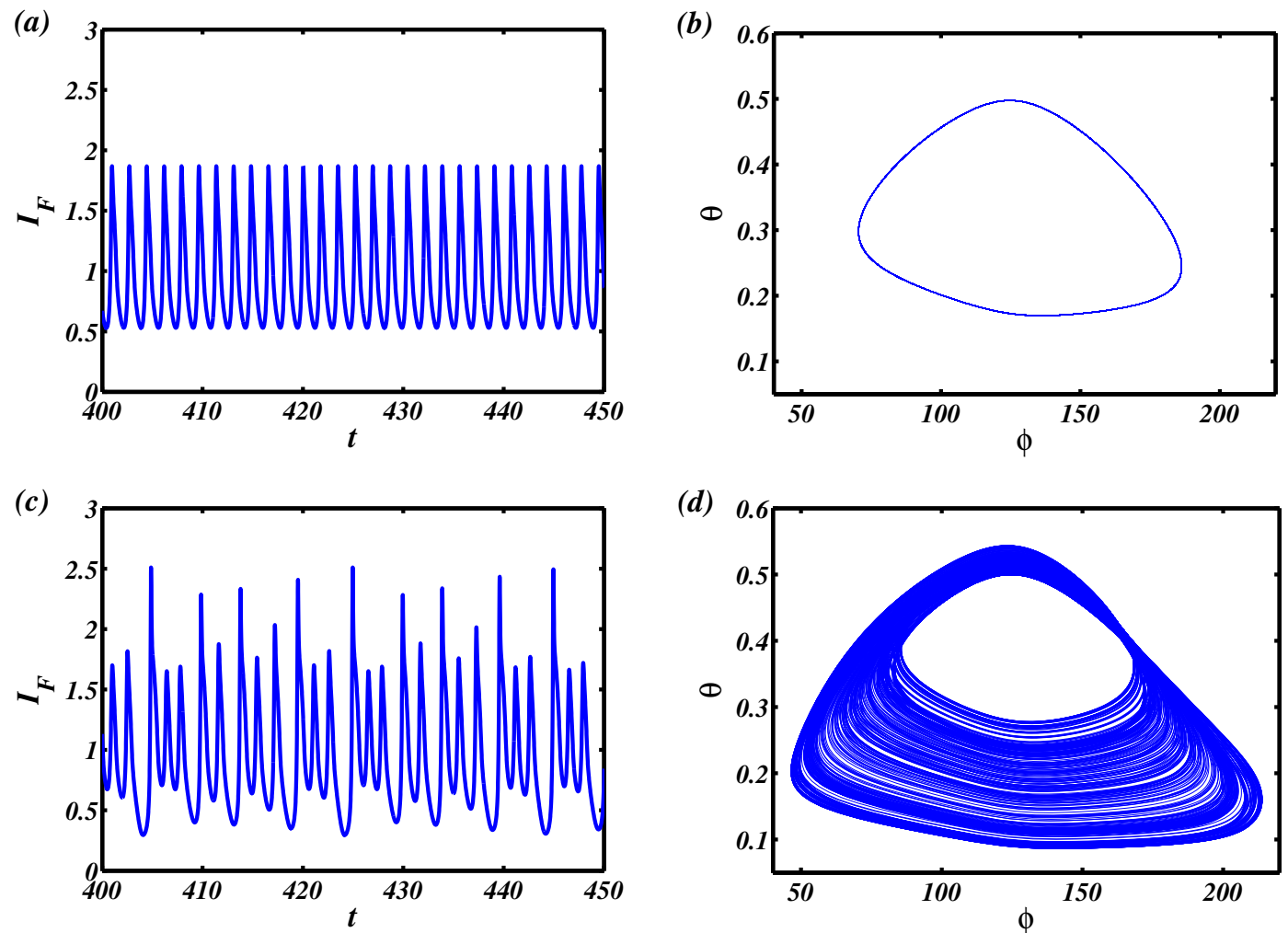

Figura 9: (a) e (c) correspondem às séries temporais periótica e cáotica para as condições de $\varepsilon=0,001, \mu=50$ e $\rho=106,7$ com dois valores distintos de $U=244$ e $U=246$. As figuras (b) e (d) são os respectivos atratores periódico e cáotico de (a) e (b). 
Observando as Figuras 8 e 9, percebe-se claramente que o modelo contempla tanto o comportamento apresentado nos voltamogramas, obtidos para moléculas orgânicas pequenas, como também o comportamento oscilatório (periódico e caótico) observado experimentalmente.

\section{I.4.1.2 Diagramas de Bifurcações}

Os primeiros diagramas de bifurcação obtidos para o modelo, podem ser visualizados na Figura 10 que descreve um conjunto de três diagramas no plano $I_{F}$ versus $U$ obtidos para $\varepsilon=0,001$ e $\mu=50$ com três valores distintos de resistências (a) $\rho=30$, (b) $\rho=50$ e (c) $\rho=180$. Nesses diagramas os pontos vermelhos correspondem aos pontos de Hopf e a linha tracejada em vermelho faz referência à região de instabilidade na solução estacionária.

(a)

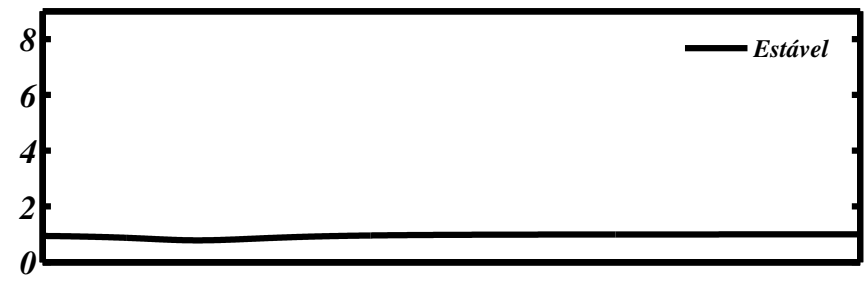

(b)

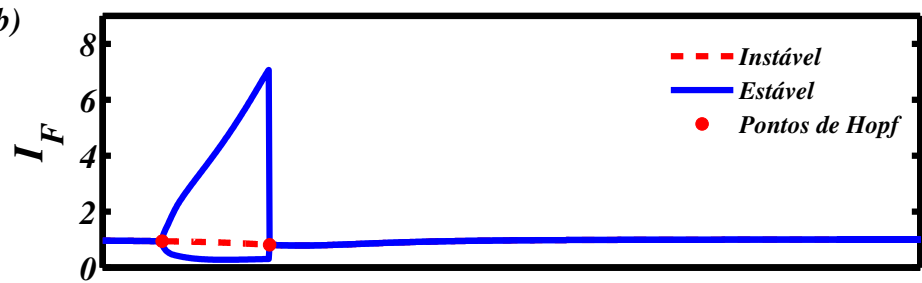

(c)

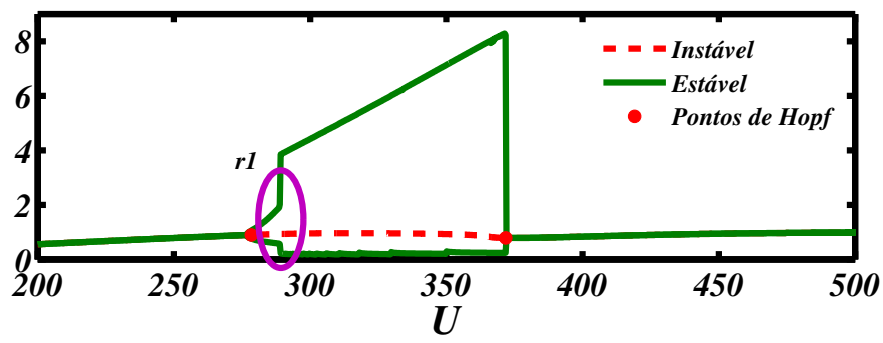

Figura 10: Diagramas de bifurcação referente aos perfis de $I_{F}$ versus $U$ para valores de $\rho$ (a) 30, (b) 50 e (c) 180 com $\varepsilon=0,001$ e $\mu=50$. 
Observando os resultados descritos na Figura 10 em termos dos perfis de $I_{F}$ versus $U$ para o primeiro valor $\rho=30$, Figura 10 (a), observamos que nessas condições o sistema não apresenta comportamento oscilatório para a variação de $U$, isso se deve a ausência de uma região de instabilidade indicada pelos pontos de Hopf. Nessa condição temos que todos os ramos da curva estacionária são acessados pelo sistema e o comportamento dinâmico é equivalente ao observado no estado estacionário. Contudo, para os valores de $\rho=50$ e $\rho=180$, Figuras 10 (b) e (c), os estados do sistema mudam devido à presença de pontos de Hopf. Nessas condições o sistema passa a apresentar comportamento oscilatório dentro dos limites indicado pelos pontos. Em ambos os casos, as oscilações nascem através de uma bifurcação supercrítica de Hopf a baixos potenciais e desaparecem no limite superior de forma abrupta. O desaparecimento rápido das oscilações no limite superior da curva de bifurcação indicado pelo segundo ponto de Hopf é causado devido à superfície do espaço de fase, no estado estacionário, ter uma curvatura na forma de $S$, tal comportamento será abordado em maiores detalhes mais adiante quando introduzirmos os diagramas de bifurcações no espaço de fase e parâmetros 3D. Como visualizado nas Figuras 10 (a), (b) e (c), os diagramas de bifurcação mostram como os perfis estacionários de $I_{F}$ versus $U$ variam de acordo com a resistência $\rho$, induzindo assim o aparecimento de ciclos limites.

Um outro ponto muito curioso nos dados exibidos na Figura 10, diz respeito à forma das bifurcações, ver Figura 10 (b) e (c). Na Figura 10 (b) temos uma variação gradativa e contínua para valores de corrente maiores na medida em que o potencial aumenta. Entretanto, o mesmo comportamento não é observado na Figura 10 (c), onde observa-se aparentemente uma quebra na continuidade da bifurcação indicada pela elipse roxa $r_{1}$. Esse comportamento pode ser melhor explicado quando construímos diagramas de órbitas levando em consideração as intensidades das oscilações entre os pontos de Hopf. Os diagramas para a Figura 10 (c) podem ser visualizados nas Figuras 11 (a) e (b), que correspondem aos diagramas de órbitas nas condições de $\rho=180$, calculado para as faixas de $200<U<500$, Figura 11 (a) e $288.5<$ $U<289.3$ Figura 11 (b) que representa uma ampliação dentro do retângulo roxo da Figura 11 (a). 

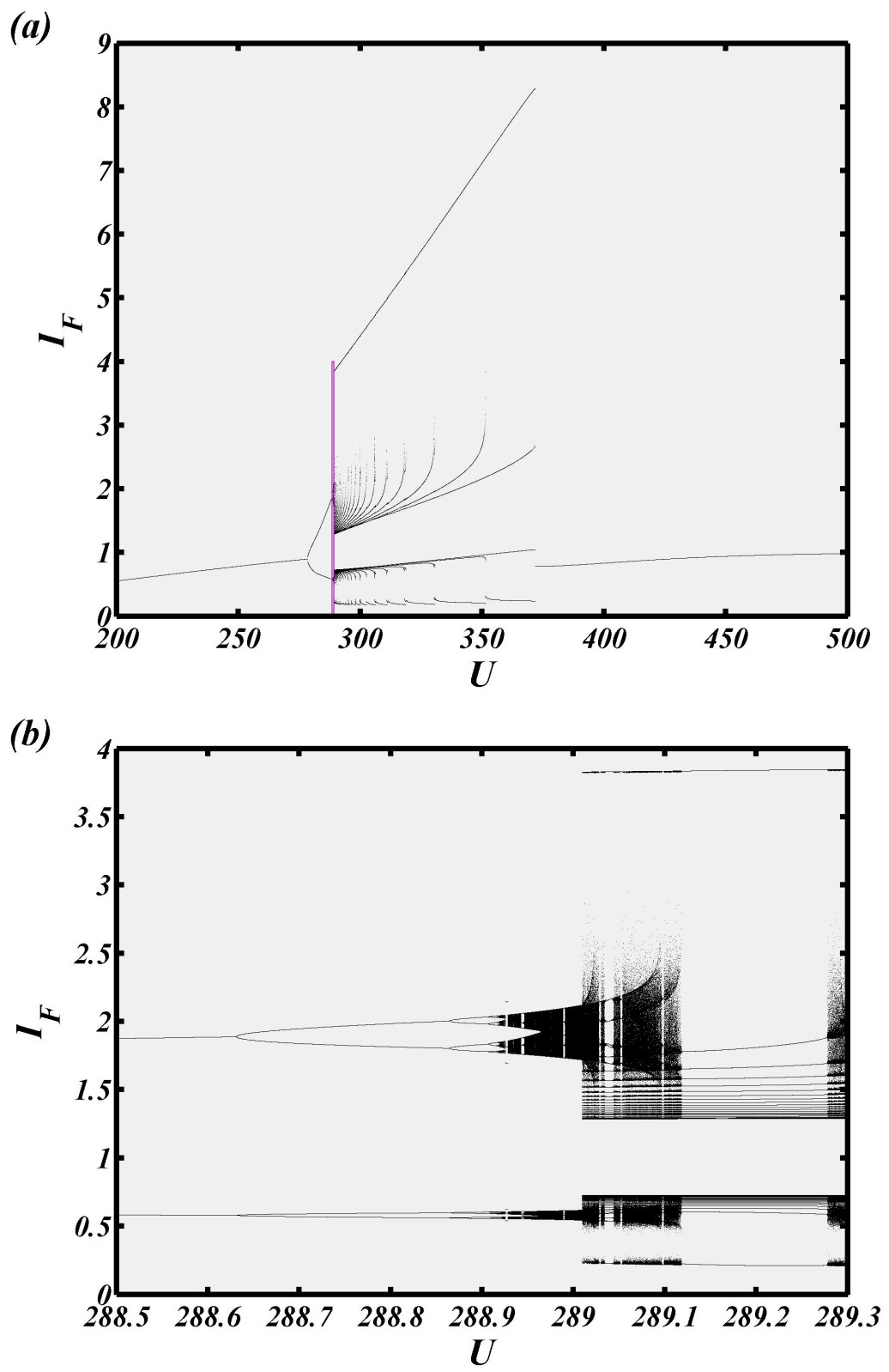

Figura 11: Diagramas de orbitas refrente a $\rho=180$, onde (a) corresponde ao diagrama na faixa de $200<U<500$ e (b) $288.5<U<289.3$.

De acordo com os resultados da Figura 11, vemos que a partir do ponto de Hopf o sistema começa a oscilar com período um, na medida em que o parâmetro $U$ aumenta a amplitude das oscilações aumenta gradativamente. Contudo, após um dado valor crítico em torno de $U=288.64$ o sistema começa a sofrer bifurcações, duplicando o período rapidamente até atingir uma região caótica, esse tipo de comportamento é visto na literatura como uma rota para o caos via duplicação de período. Quando o sistema passa pela "janela caótica" ele volta a apresentar comportamento periódico, porém o período apresentado é diferente em relação ao 
comportamento anterior, gerando uma triplicação de período que desloca-se rapidamente para a nova janela. Esse tipo de comportamento causa uma variação abrupta dos períodos e das intensidades no interior da bifurcação gerando o comportamento observado na Figura 10 (c). Uma forma de analisar o comportamento periódico do sistema é por meio dos gráficos de períodos que serão discutidos posteriormente.

Até agora foram discutidos os perfis dos gráficos de bifurcação para $I_{F}$ versus $U$ de forma simplificada. Contudo, foi demonstrando que a variação na resistência total induz o aparecimento de ciclos-limite, assim como uma mudança na morfologia apresentada pelas bifurcações nas Figuras 10 (b) e (c), tais modificações estão relacionadas ao tipo de rota assumida pelo sistema. No caso da Figura 10 (b) revelou apenas um aumento na intensidade das oscilações, por outro lado, na Figura 10 (c) a rota dinâmica observada foi a duplicação de período antes das janelas caóticas. Entretanto, para uma análise mais aprofundada do comportamento dinâmico do sistema se faz necessário avaliar o diagrama de parâmetros no plano $\rho$ versus $U$, ver Figura 12. A motivação de se trabalhar com esse plano de parâmetros se deve ao fato deles serem facilmente compatíveis aos obtidos experimentalmente [68, 69].

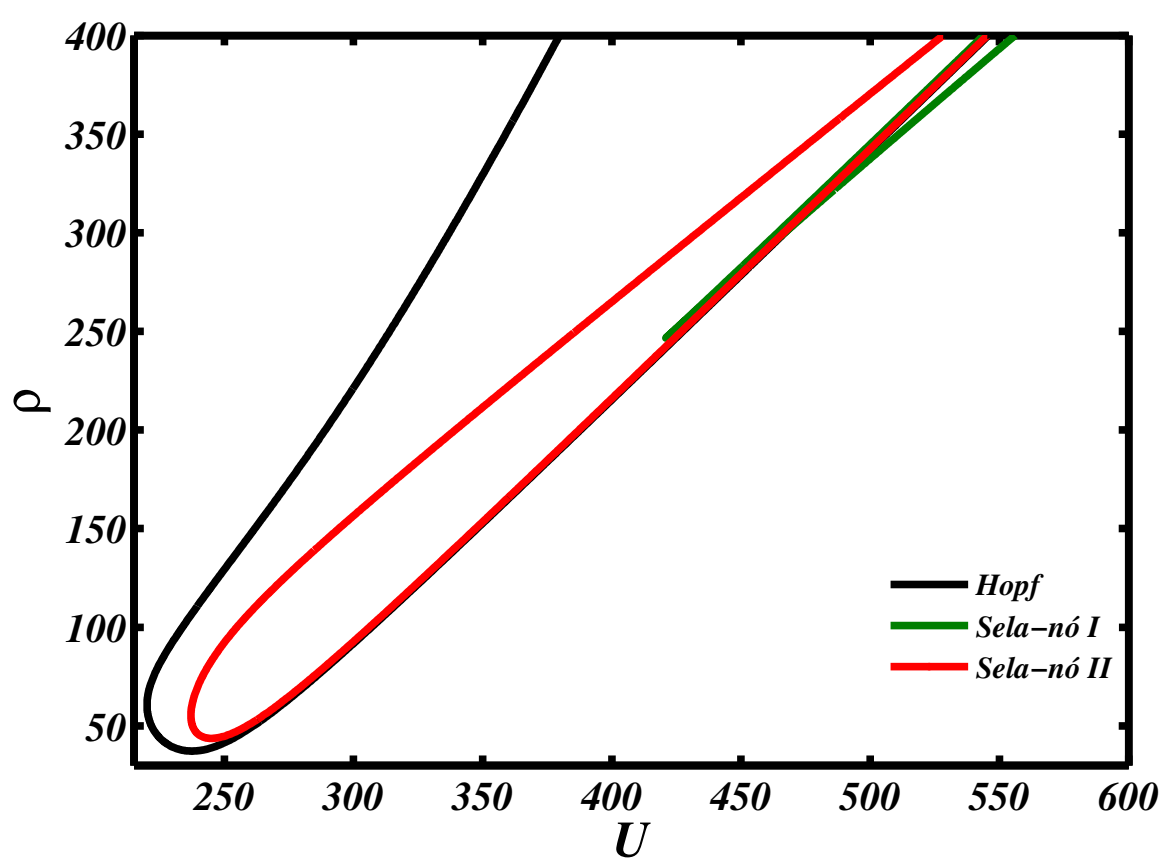

Figura 12: Curvas de bifurcação no plano de parâmetros $\rho$ versus $U$ para $\varepsilon=0,001$ e $\mu=50$. 
A Figura 12 descreve um típico diagrama no plano dos parâmetros $\rho$ versus $U$, onde a curva em preto corresponde à bifurcação de Hopf. Já as curvas em vermelho e verde fazem referência às bifurcações de sela-nó tipo I e II. Nesse tipo de diagrama pode-se distinguir, no interior da bifurcação, três tipos distintos de regiões que são relacionadas ao de valor assumido pelos autovalores no estado estacionário, onde a região delimitada pela linha preta faz referência aos autovalores complexos conjugados com parte real diferente de zero e um autovalor real. Já para as duas curvas em vermelho e verde, temos autovalores reais com (a) sinais $(--+)$ indicando um comportamento de sela-nó tipo I e (b) com sinais $(-++)$ relativos ao comportamento de sela-nó tipo II. Um resumo da dinâmica pontual observada para os pontos fixos num conjunto formado por três equações diferenciais não-lineares é descrito na Figura 13.

(a)

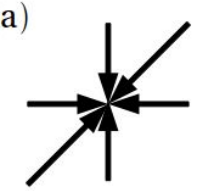

Ponto fixo estável

$\left(-\lambda_{1},-\lambda_{2},-\lambda_{3}\right)$

(e)

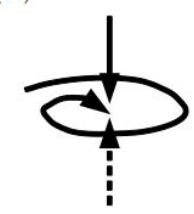

Foco estável $\left(-\lambda_{1},-\sigma \pm \omega \mathrm{i}\right)$ (b)

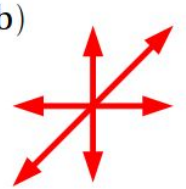

Ponto fixo instável $\left(\lambda_{1}, \lambda_{2}, \lambda_{3}\right)$

(f)

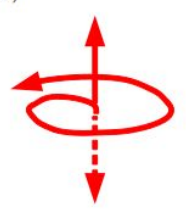

Foco instável $\left(\lambda_{1}, \sigma \pm \omega \mathrm{i}\right)$ (c)

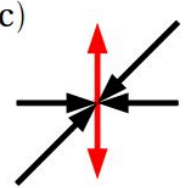

Sela-nó tipo I

$\left(\lambda_{1},-\lambda_{2},-\lambda_{3}\right)$

(g)

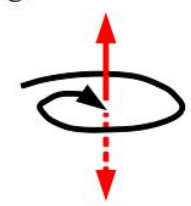

Sela foco tipo I

$\left(\lambda_{1},-\sigma \pm \omega \mathbf{i}\right)$ (d)

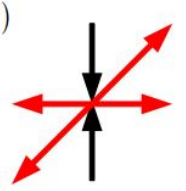

Sela-nó tipo II $\left(-\lambda_{1}, \lambda_{2}, \lambda_{3}\right)$

(h)

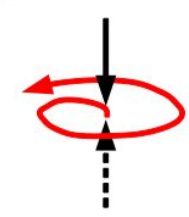

Sela foco tipo II $\left(-\lambda_{1}, \sigma \pm \omega \mathrm{i}\right)$

Figura 13: Esboço das possíveis trajetórias adotadas pelos pontos fixos no espaço de fase tridimensional. Nesse esboço são mostradas duas possíveis configurações para os pontos de sela-nó (c) tipo I e (d) tipo II, assim como os pontos de sela-foco (g) tipo I e (h) tipo II.

Avaliando o cenário apresentado na Figura 12 junto com a informação extraída da Figura 11. Emerge um questionamento interessante com respeito ao tipo de influência exercida pelos pontos fixos sobre a dinâmica global do sistema. Sendo essa uma das maiores complicações encontradas em estudos relacionando às transcições do comportamento dinâmico obser- 
vado em equações não-lineares. Segundo Gaspard e Nicolis [70] o comportamento dinâmico pode ser abordado por dois caminhos diferentes: o primeiro reduz alguns aspectos da dinâmica global para um problema local; o segundo tem um caráter bem mais audacioso e procura estabelecer regras para organizar o comportamento dinâmico observado em sistemas afastados do equilíbrio. Um bom exemplo de como essas regras podem ser estabecidas é a rota para o caos via cenário de Feigenbaum [71] mostrando uma cascata de bifurcações do tipo flip ou duplicação de período para sistemas discretos.

De acordo com Gaspard e colaboradores [70-73] uma outra regra para descrever o comportamento complexo como oscilações de período misto e caos pode ser obtida por meio do estudo das órbitas homoclínicas ${ }^{\dagger}$ em sistemas dinâmicos. Tais órbitas podem ser causadas pela natureza dos autovalores associados ao campo vetorial nos pontos fixos. De acordo com Wiggins [74] existem duas formas para se obter um comportamento homoclínico associado a três autovalores:

1. Pontos de sela-nó que podem ser divididos em dois tipos distintos dados por: (a) sela-nó tipo I e (b) sela-nó tipo II. Esses dois tipos de pontos podem ser encontrados no gráfico de parâmetro exibido na Figura 12. Uma forte característica exibida pelas órbitas homoclínicas referente aos pontos de sela-nó é mostrado no Teorema de Gavrilov e Shilnikov [74] para um sistema genérico na forma de:

$$
\begin{aligned}
& \frac{d x}{d t}=\lambda_{1} x+f_{1}(x, y, z, \eta) \\
& \frac{d y}{d t}=\lambda_{2} x+f_{2}(x, y, z, \eta) \\
& \frac{d z}{d t}=\lambda_{3} x+f_{3}(x, y, z, \eta)
\end{aligned}
$$

\footnotetext{
$\dagger$ Órbitas homoclínicas ocorrem quando a órbita percorrida por um ponto de sela $P$ inicia-se e termina nele mesmo. Já órbitas heteroclínicas acontecem quando a trajetória inicia-se em um ponto de sela $P_{1}$ e termina em outro ponto de sela $P_{2}$ [13].
} 
sendo $(x, y, z, \eta) \in \mathbb{R}^{4}$, onde o termo $\eta$ representa um parâmetro genérico e $\lambda_{1}, \lambda_{2}$ e $\lambda_{3}$ são os autovalores reais do sistema. Segundo esse teorema é possível relacionar os valores correspondentes à bifurcação de sela-nó com os valores correspondentes à bifurcação de duplicação de período. Tal característica se mostra muito interessante, pois sequências de duplicação de período antes e depois das janelas caóticas foram observadas no diagrama de órbitas, Figura 11, para o diagrama de bifurcação da Figura 10 (c).

2. Pontos de sela-foco que também podem ser divididos em dois tipos distintos de comportamento dado por: (a) sela-foco tipo I, onde os autovalores são definidos como $\left(\lambda_{1},-\sigma \pm\right.$ $\omega i)$ e (b) que corresponde ao ponto de sela-foco tipo II dado pelos autovalores $\left(-\lambda_{1}, \sigma \pm\right.$ $\omega i)$. Os fenômenos dinâmicos observados para um ponto de sela-foco são conhecidos como fenômenos de Shilnikov e podem ser definidos a partir do sistema de equações abaixo:

$$
\begin{gathered}
\frac{d x}{d t}=\sigma x-\omega y+P(x, y, z) \\
\frac{d y}{d t}=\omega x+\sigma y+Q(x, y, z) \\
\frac{d z}{d t}=\lambda z+D(x, y, z)
\end{gathered}
$$

onde, $\lambda$ e $\sigma \pm \omega i$ são os autovalores para um ponto de sela-foco. Já $P, Q$ e $D$ são as funções analisadas para $x, y$ e $z$ quando a primeira derivada de $x, y$ e $z$ é zero. Segundo Gaspard e colaboradores [72] a condição para que o sistema apresente comportamento homoclínico de sela-foco descrito pelo Teorema de Shilnikov [75] é dada por:

$$
\begin{gathered}
v=\left|\frac{\sigma}{\lambda}\right| \\
v<1
\end{gathered}
$$


O Teorema de Shilnikov fala que: se $|\sigma|<\lambda$, então o fluxo dado por $\chi_{t}$ pode ser perturbado para $\chi_{t}^{\prime}$, tal que, $\chi_{t}^{\prime}$ tem uma órbita homoclínica $\gamma^{\prime}$ perto de $\gamma$ e o mapa de retorno de $\gamma^{\prime}$ para $\chi_{t}^{\prime}$ tem um conjunto contável de ferraduras (horseshoes).

Além da condição descrita pela Equação 37, temos que as perturbações para sistemas homoclínicos causam um conjunto quase infinito de tangências homoclínicas ${ }^{\dagger}$ que são seguidas por bifurcações de duplicação de período. As órbitas periódicas devem ser atraídas se a condição de Shilnikov for menor que 0,5, como descrito pela Equação 38.

$$
v<0,5
$$

O cenário homoclínico para sistemas não-lineares obtidos por meio dos pontos de selafoco, cenário de Shilnikov, tem sido discutido por Gaspard e colaboradores [72, 73], assim como Arneodo et al. [76], Glendinning e Sparrow [77]. Segundo eles, a relação das trajetórias homoclínicas e comportamento complexo não periódico explorado em sistemas modelos tais como o sistema de Lorenz e Rössler [78, 79], pode ser entendida por meio da perspectiva de Shilnikov, Equação 37. Contudo, vamos observar mais adiante que o comportamento dinâmico não periódico pode surgir na ausência da condição de Shilnikov.

Uma importante característica relatada nos artigos de Koper et al. [80, 81] com relação à condição de Shilnikov observada em experimentos e modelos teóricos é que nem todas as condições do Teorema de Shilnikov são satisfeitas, tal comportamento foi chamado por Koper de cenário homoclínco incompleto. Segundo Koper, esse cenário causa uma contração muito forte no espaço de fase fazendo com que os estados caóticos sejam espremidos em uma linha e o cenário caótico-periódico aparece como uma sequência de Farey.

Para discutirmos melhor o cenário de Shilnikov e o proposto por Koper et al. [80, 81], temos que levar em consideração a forma superficial do espaço de fase e parâmetros no estado estacionário, assim como a estabilidade do sistema apresentada pelos pontos fixos no espaço de

\footnotetext{
†Tangência homoclínica ocorre quando o manifold estável torna-se tangente a um manifold instável por meio de um valor crítico de um parâmetro. Um ponto muito interessante a respeito das tangências homoclínicas é o fato delas serem extremamente instáveis [73].
} 
parâmetros. Para tanto foram calculados os diagramas de bifurcação 3D dados pelas Figuras 14 (a) e (b), além dos diagramas de estabilidade pontual exibidos nas Figuras 17 (a) e (b).

(a)

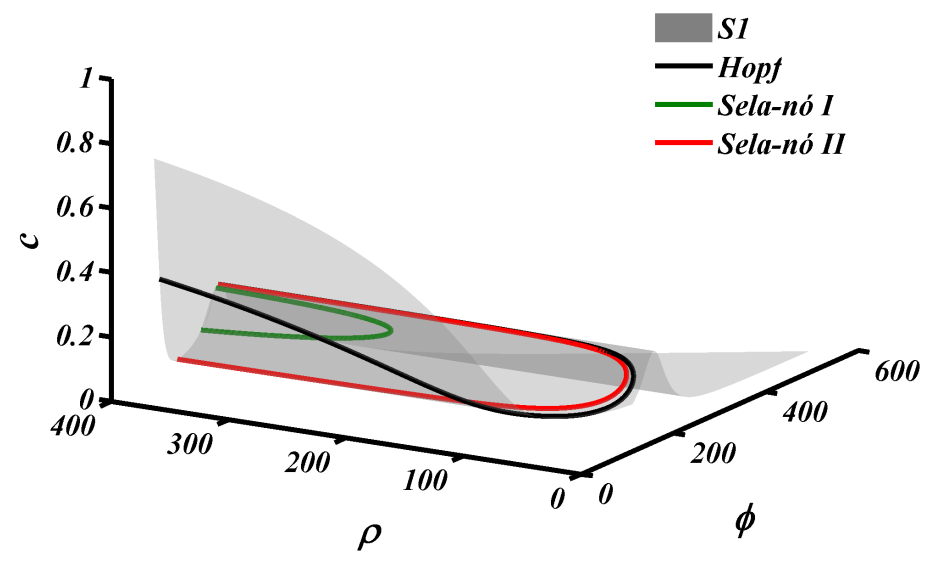

(b)

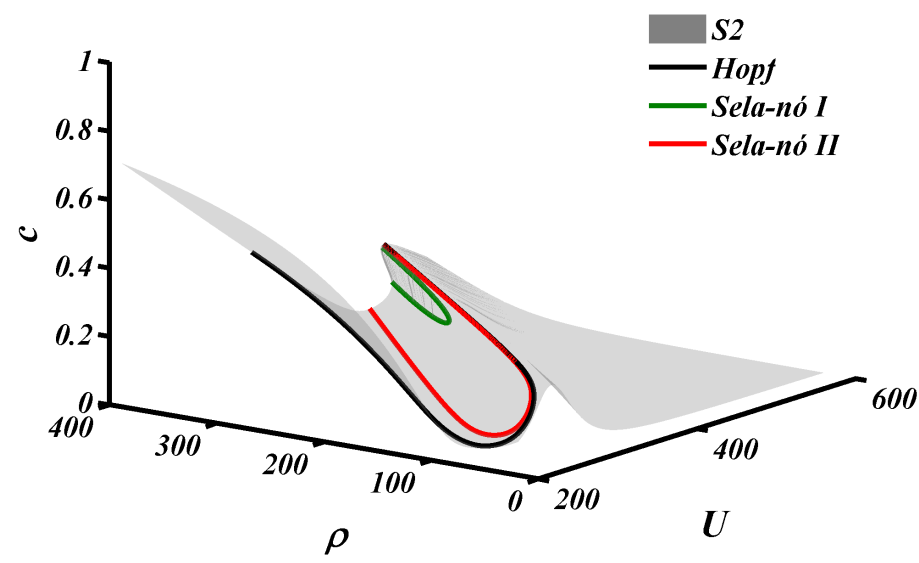

Figura 14: Diagramas de bifurcação 3D para (a) plano de variáveis e parâmetro $(\phi, c, \rho)$ e (b) plano de parâmetros e variável $(\rho, U, c)$, nas condições de $\varepsilon=0,001$ e $\mu=50$.

Observando a Figura 14 (a) e (b) que corresponde aos diagramas de bifurcação 3D no espaço de fase das variáveis e parâmetro $(\phi, c, \rho)$, assim como no espaço dos parâmetros e variável $(\rho, U, c)$, respectivamente. Nota-se que a superfície dada por $S_{1}$ e $S_{2}$, Figuras 14 (a) e (b), têm a forma de $S$. Um ponto intrigante associados às curvas estacionárias com essa forma é a presença de um polinômio de ordem três que pode está explícito ou implícito em uma das equações diferenciais. Tal forma tem como característica a presença de duas escalas de tempo distintas formando uma região rápida denominada de fast manifold e uma região lenta chamada de slow manifold. De acordo com Desroches e Jeffrey $[82,83]$ esse comportamento pode ser relacionado aos fenômenos dinâmicos de oscilações amortecidas e ciclos de Canard, que corres- 
ponde à explosão numa cascata de ciclos crescentes ocorrendo em um intervalo de parâmetros extremamente pequeno, isso implica que tais fenômenos são difíceis de serem observados, quer em situações experimentais ou numéricas. Tais características também foram relatadas por Koper e Gaspard [81] na forma de um princípio de reinjeção que favorece as condições para a existência do comportamento complexo como oscilações de modo misto e caos.

Nas Superfícies $S_{1}$ e $S_{2}$, Figura 14 (a) e (b), foram adicionadas as curvas de bifurcações para Hopf, sela-nó I e II indicadas pelas linhas preta, verde e vermelha respectivamente. Note que as curvas de Hopf e sela-nó I têm suas superfícies praticamente paralelas na condição de $\rho>150$, Figura (a) e $\rho>150$ com $U>300$, Figura (b). Essa situação é muito interessante, pois se aplicarmos o princípio de reinjeção temos que a reinjeção dos pontos de sela-nó I e II será na superfície instável referente aos pontos de sela-foco. Outra característica importante é o fato do ramo direito das curvas de bifurcações estarem praticamente na mesma região de instabilidade. Isso indica que sempre no final dos diagramas de bifurcação obtidos para uma variação crescente do parâmetro $U$ vamos ter um ponto de sela-nó I ou II muito próximo do ponto de Hopf. Essa proximidade faz com que as órbitas referentes ao comportamento de Hopf sejam atraídas pelas órbitas dos pontos de sela que direcionam o fluxo para a outra região da curva. Para visualizar melhor esse comportamento foram calculadas duas curvas no estado estacionário para dois valores distintos de $\rho$ e coletados os pontos fixos de Hopf, sela-nó I e II referentes ao ramo direito da curva, indicado pelo círculo azul nas Figuras 15 (a) e (b). Os valores das condições em que os pontos são encontrados são exibidos na Tabela 3, onde a condição (a) refere-se ao valor de $\rho=100$ e (b) $\rho=280$.

Tabela 3: Condições para construção das órbitas referente a Figura 15.

\begin{tabular}{|c|c|c|c|c|c|}
\hline & & $\phi$ & $c$ & $\theta$ & $U$ \\
\hline \multirow{3}{*}{ (a) $\rho=100$} & \multirow{3}{*}{$\begin{array}{l}\text { Hopf } \\
\text { Sela-nó I } \\
\text { Sela-nó II }\end{array}$} & 227,917 & 0,210 & 0,000 & 306,915 \\
\hline & & - & - & - & - \\
\hline & & 226,871 & 0,208 & 0,000 & 306,109 \\
\hline \multirow{3}{*}{ (b) $\rho=280$} & Hopf & 231,460 & 0,215 & 0,000 & 451,188 \\
\hline & Sela-nó I & 222,072 & 0,193 & 0,000 & 448,064 \\
\hline & Sela-nó II & 230,811 & 0,215 & 0,000 & 450,706 \\
\hline
\end{tabular}


As Figuras 15 (a) e (b) descrevem o comportamento para as curvas estacionárias em duas situações distintas, onde a Figura 15 (a), referente ao valor de $\rho=100$ corresponde a uma situação em que a reinjeção dos pontos de Hopf e sela-nó ocorrem na região estável da curva estacionária. Já a Figura 15 (b), valor de $\rho=280$, realça o processo de reinjeção dos pontos na região instável da curva. As Figuras 15 (a1) e (b1) são ampliações do comportamento dinâmico próximo às vizinhanças dos pontos de Hopf e Sela-nó do tipo II referente ao ramo direito da curva estacionária, com suas órbitas indicadas pelas linhas azul e verde respectivamente. As séries temporais para as condições do ponto de Hopf e sela quando $\rho=100$ e $\rho=280$ são mostradas nas Figuras 15 (a2), (a3), (b2) e (b3) em função da variável $\phi$.

(a)

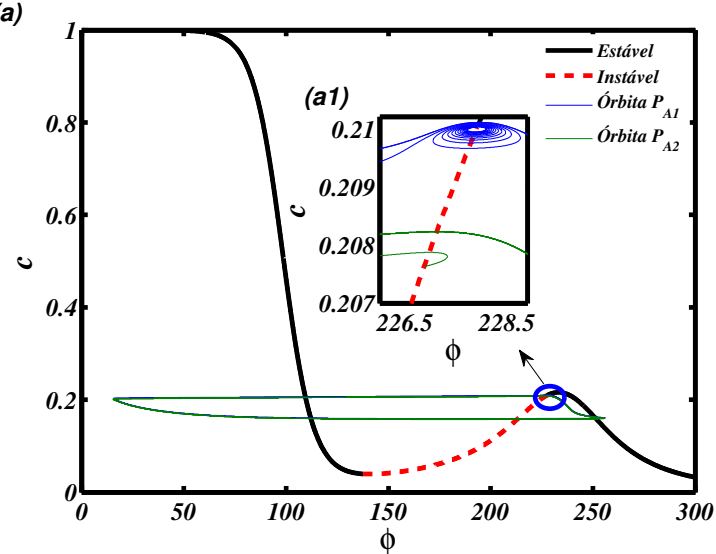

(b)

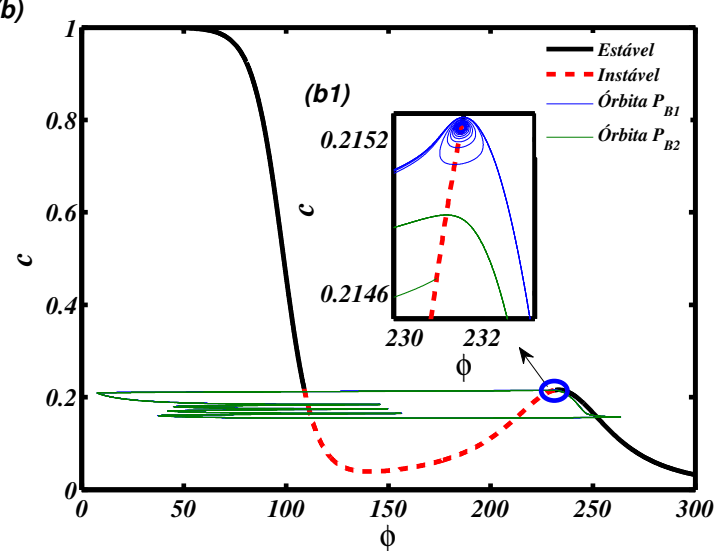

(a2)
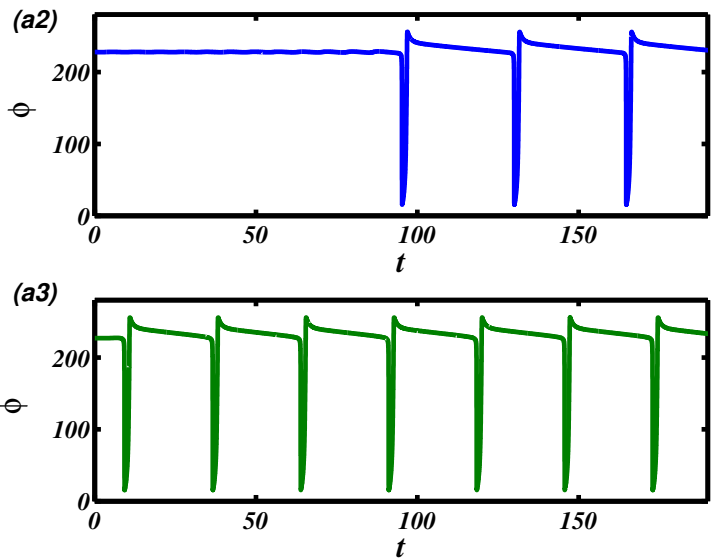

(b2)

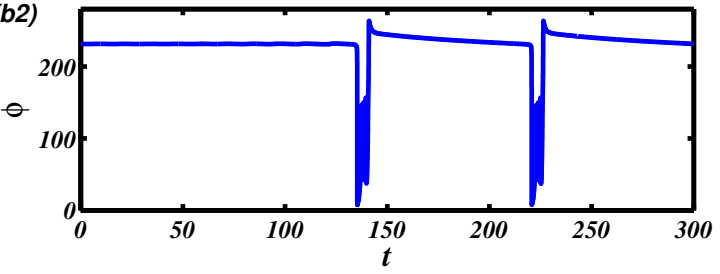

(b3)

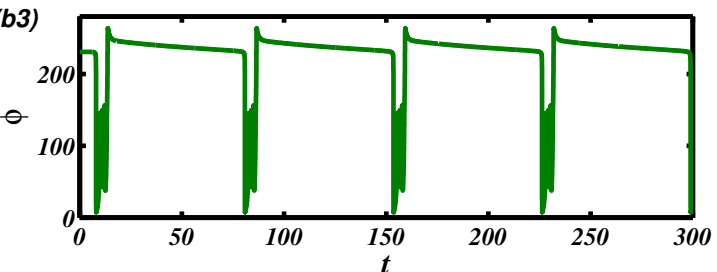

Figura 15: Curvas estacionárias para os valore de (a) $\rho=100$ e (b) $\rho=280$ caculadas para os valores de $\varepsilon=0,001$ e $\mu=50$. As séries temporais referentes às órbitas $P_{A 1}, P_{A 2}, P_{B 1}$ e $P_{B 2}$ são mostradas em (a2), (a3), (b2) e (b3) respactivamente. 
Analisando a Figura 15 (a) e (a1) percebe-se que a órbita para o ponto de Hopf iniciase com pequenos ciclos que vão aumentado no tempo na direção do ponto de sela-nó II até atingir uma condição crítica, em que o fluxo explode rapidamente na direção da região estável a esquerda da curva estacionária que logo em seguida retorna as proximidades do local original completando assim um ciclo. Um dado interessante aqui é que a órbita não retorna exatamente ao ponto de origem. Esse comportamento pode ser interpretado como a formação de uma bifurcação homoclínica, onde um ciclo limite move-se em direção ao ponto de sela, tornadose uma órbita homoclínica. Nesse tipo de bifurcação temos que os ciclos limites atingem as regiões de domínio dos pontos de sela para um valor crítico do parâmetro formando assim uma órbita homoclínica.

Quando voltamos à atenção para o ponto de sela-nó II na Figura 15 (a), percebe-se que a órbita apresenta praticamente o mesmo comportamento observado no ponto de Hopf da Figura 15 (a). A única diferença é com relação ao fluxo na volta ao ponto original, onde para o ponto de Hopf o percurso de volta é mais lento em relação ao percurso do ponto de sela, essa característica indica que processos diferentes ocorrendo nas regiões de slow manifold podem ser diferenciados pela escala de tempo envolvida. Tal comportamento pode ser visto nas séries temporais referentes aos pontos de Hopf e sela-nó na Figura 15 (a2) e (a3) respectivamente.

Ao analisar os dados da Figura 15 (b) referente ao valor de $\rho=280$, notamos que as órbitas para os pontos de Hopf e sela-nó do tipo II apresentam um comportamento oscilatório na região de reinjeção correspondente ao lado esquerdo da curva estacionária. Note que as únicas diferenças entre as reinjeções nas regiões estacionárias são as suas instabilidades, onde a curva calculada para o valor de $\rho=100$ a região de reinjeção é estável, já para o valor de $\rho=280$ temos instabilidade. Contudo, o critério da reinjeção ocorrer fora ou dentro das regiões de instabilidade não pode ser utilizado para justificar o comportamento oscilatório de ordem mista ou caos. Apesar disso seu entendimento é crucial para explicar o comportamento dinâmico manifestado no interior das bifurcações quando a condição de Shilnikov não é satisfeita.

Um dado interessante a respeito do comportamento dinâmico para o parâmetro $\rho=$ 280 é que toda variação feita no sistema próxima dos pontos de sela-nó do tipo I e II e interior de suas bifurcações vão apresentar órbitas de reinjeção. Esse comportamento pode ser observado 
nos atratores das Figuras 16 (a), (b), (c) e (d) que mostram um conjunto formado por oito órbitas na qual quatro correspondem aos pontos de Hopf com suas condições expressas na Tabela 4 (a) e sela-nó expressas na Tabela 4 (b).

Tabela 4: Condições para construção das oito órbitas referente às Figuras 16 (a), (b), (c) e (d), onde as órbitas para os pontos de Hopf correspondem a Tabela (a). Já as órbitas de sela-nó do tipo I e II Tabela (b).

(a)

\begin{tabular}{|c|c|c|c|c|}
\hline \multirow[b]{2}{*}{ Órbitas } & \multicolumn{4}{|c|}{ Hopf } \\
\hline & 1 & 2 & 3 & 4 \\
\hline$\phi \rightarrow$ & 108,862 & 112,201 & 120,209 & 133,205 \\
\hline$c \rightarrow$ & 0,218 & 0,159 & 0,078 & 0,042 \\
\hline$\theta \rightarrow$ & 0,901 & 0,851 & 0,649 & 0,230 \\
\hline$U \rightarrow$ & 327,865 & 347,803 & 378,261 & 401,464 \\
\hline
\end{tabular}

(b)

\begin{tabular}{l|c|c|c|c|}
\multicolumn{4}{c}{ Sela-nó I e II } \\
\cline { 2 - 5 } Órbitas & 5 & 6 & 7 & 8 \\
\cline { 2 - 5 }$\phi \rightarrow$ & 145,221 & 178,304 & 206,542 & 215,110 \\
\cline { 2 - 5 }$c \rightarrow$ & 0,0395 & 0,065 & 0,132 & 0,166 \\
\cline { 2 - 5 }$\theta \rightarrow$ & 0,052 & 0,000 & 0,000 & 0,000 \\
\cline { 2 - 5 }$U \rightarrow$ & 414,161 & 439,906 & 449,390 & 448,723 \\
\cline { 2 - 5 } & & &
\end{tabular}

A Figura 16 (a) e (b) descrevem os atratores nas condições de Hopf para o espaço de fase dado por $(\phi, c)$, Figura 16 (a) e o espaço de fase $(\phi, \theta)$, Figura 16 (b). As Figuras 16 (a1) e (b1) são ampliações do atrataor referente à primeira órbita localizada no interior dos círculos roxos.

Observando os gráficos da Figura 16 (a) e (b), percebe-se que às órbitas 1 e 2 apresentam um comportamento bem diferente em comparação às órbitas 3 e 4 que possuem um comportamento praticamente idêntico. As órbitas 1 e 2 são muito compactas e limitads a uma região pequena do espaço de fase. Tal comportamento pode ser explicado simplesmente considerando a distância em relação ao parâmetro $U$ dos pontos de Hopf 1 e 2 com relação ao primeiro pondo de sela-nó dados pela condição da órbita 5, ver Tabela 4 (a) e (b). Tal proximidade com os pontos de sela-nó faz com que o fluxo seja redirecionado para a região de slow manifold. Uma vez nessa região, o fluxo segue uma órbita homoclínica e o processo de reinjeção passa a atuar. Quando observamos os atratores obtidos nas condições de sela-nó, ver Figura 4 (c) e (d), percebemos melhor essa influência do princípio de reinjeção, note que todos 
os atratores dentro das bifurcações de sela-nó do tipo I e II apresentam praticamente o mesmo comportamento em relação à região de slow manifold.
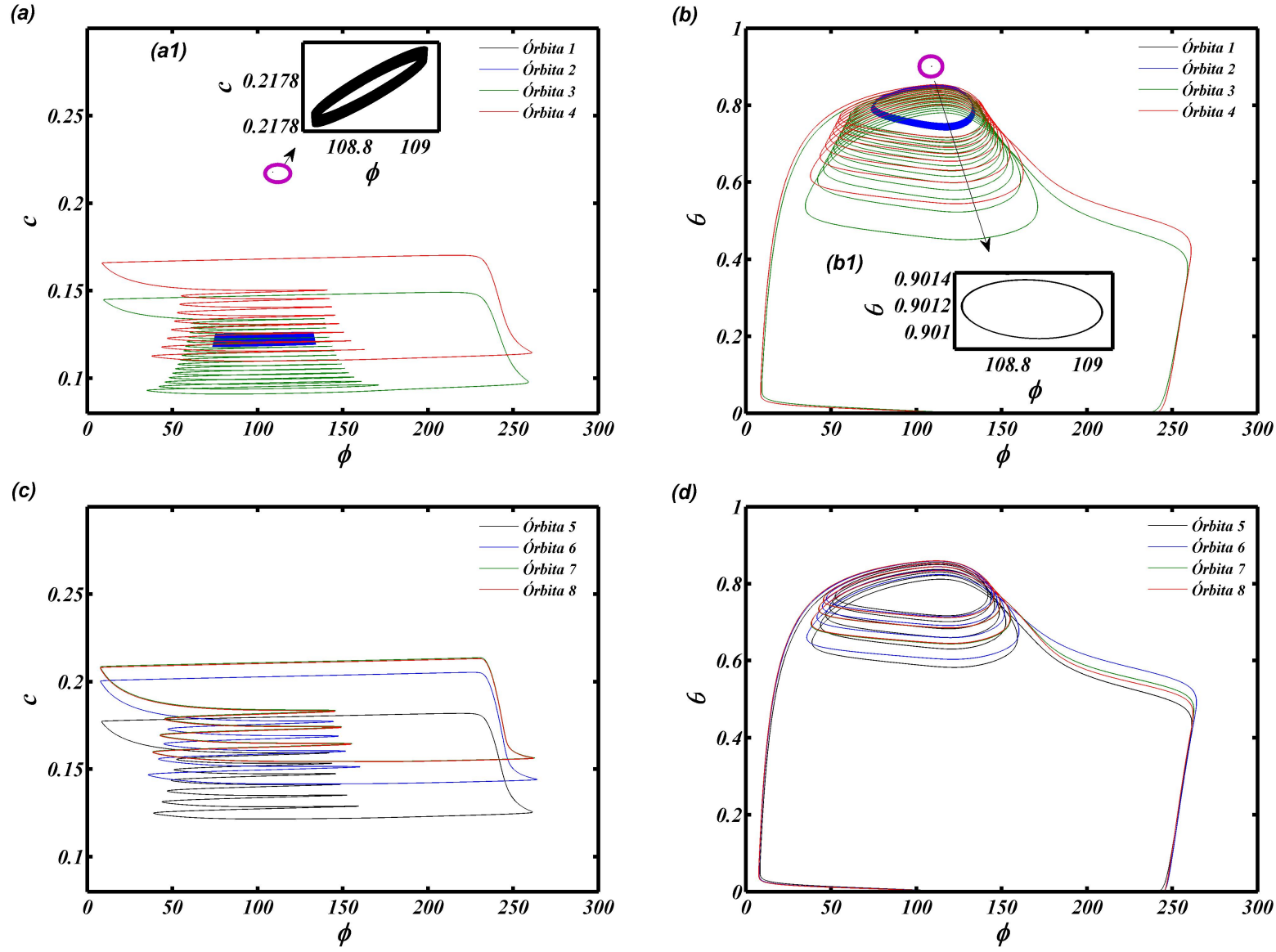

Figura 16: Órbitas nas condições de Hopf, sela-nó I e II calculadas para o parâmetro $\rho=280$, $\varepsilon=0,001$ e $\mu=50$, onde (a) e (b) correspondem às órbitas referentes aos pontos de Hopf no plano de fase $(\phi, c)$ e $(\phi, \theta)$. Já (c) e (d) referem-se às órbitas dos pontos de sela-nó no plano de fase $(\phi, c)$ e $(\phi, \theta)$.

Tanto os diagramas das curvas estacionárias, Figuras 15 (a) e (b), quando a análise das órbitas dos atratores, Figuras (a), (b), (c) e (d), são ferramentas importantes para o diagnóstico do comportamento dinâmico fora da condição de Shilnikov. Contudo, ainda fica muito difícil de diagnosticar todo o espaço de parâmetros para uma variação de $\rho$ versus $U$. Uma das formas de fazer essa análise é encontrando todas as condições com relação ao comportamento de Shilnikov, Equações 37 e 38. Para isso foi proposto um gráfico que descreve o comportamento da dinâmica pontual com base nos autovalores obtidos pelo modelo. Esse gráfico pode ser visto na Figura 17 (a), sendo que a Figura 17 (b) corresponde a uma versão do gráfico de parâmetros onde não é feita a correção do eixo $x$ em relação a $U$, que nesse caso vamos adotar a nomen- 
clatura de $U$ não corrigido ao longo desse texto. A não correção do parâmetro $U$ faz com que a superfície em forma de $S$ seja esticada mostrando alguns comportamentos que são sobrepostos na forma usual de análise.

As Figuras 17 (a) e (b) foram calculadas para as condições de parâmetros referentes à Figura 12, onde as cores no diagrama de estabilidade pontual mostradas no mapa de cor ao lado de cada figura correspondem ao tipo de estabilidade adquirida pelo pondo fixo. As condições de sela-foco I e II, Shilnikov I e II, assim como sela-nó I e II são relacionados à direção do fluxo assumido pelos pontos no espaço de fase. Já o critério de $0,5<v<1$ e $0<v<0,5$ na condição de Shilnikov é referente ao critério fornecido pelas Equações 37 e 38.

De acordo com Gaspard et al. [72], nas condições de Shilnikov referente à Equação 38 temos que o resultado mostra a complexidade das bifurcações relacionadas aos atratores periódicos perto das órbitas homoclínicas de Shilnikov, sugerindo assim a possibilidade da coexistência de muitos atratores. Outro ponto também interessante levantado por Wiggins [74] a respeito da condição 38 é o fato do tipo de estabilidade apresentada pelos pontos fixos do mapa de Poincaré obtido pela análise do conjunto de Equações 33, 34 e 35, que segundo Wiggins a análise da matriz jacobiana obtida para o mapa possui uma área expansiva nessas condições.
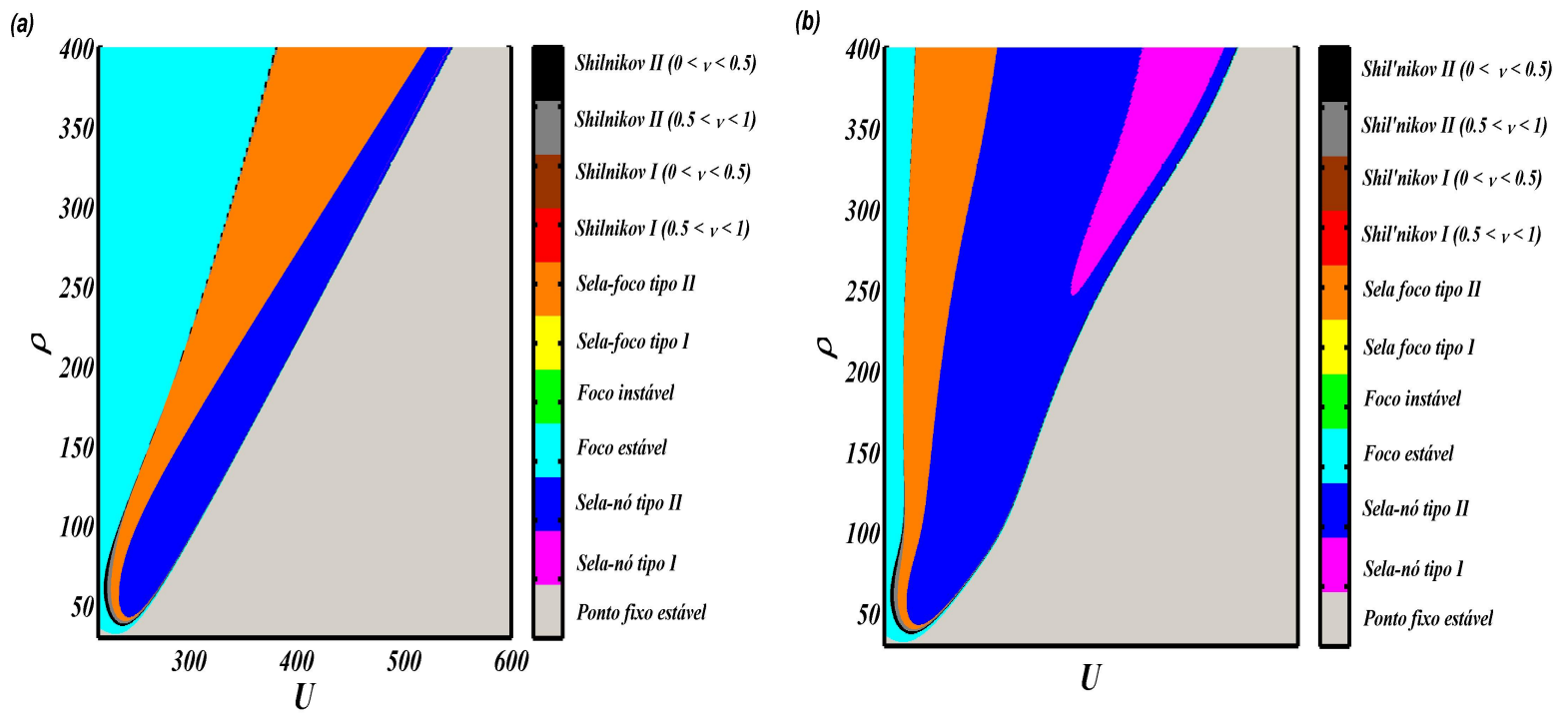

Figura 17: Diagrama de estabilidade pontual para (a) espaço de parâmetro $\rho$ versus $U$ e (b) diagrama de estabilidade pontual deformado em relação ao eixo $U$, obtidos para os valores de $\varepsilon=0,001$ e $\mu=50$. 
Observando as Figuras 17 (a) e (b), notamos que a região no interior da bifurcação de Hopf é dominada basicamente por três tipos distintos de estabilidades indicadas pelas cores laranja, rosa e azul escuro que correspondem às estabilidades de sela-foco II, sela-nó I e II respectivamente. Note que além das três regiões, também encontramos duas regiões bem comprimidas logo no início do diagrama de bifurcação para valores de $30<\rho<100$ e $215<$ $U<250$ aproximadamente, essas regiões referem-se aos valores de Shilnikov II nas condições de $0<v<0,5$ e $0,5<v<1$, representadas nas cores preta e cinza escuro respectivamente. Um ponto interessante a ser relatado a respeito do comportamento dinâmico dessas regiões é que devido a sua compressão não deve ser possível observar comportamento oscilatório complexo como oscilações de ordem mista e caos, assim podemos falar que para as condições em que as Figuras 17 (a) e (b) foram obtidas o comportamento dinâmico não sofre influência da condição de Shilnikov. Essa afirmação é muito forte, pois implica em dizer que outro comportamento dinâmico está atuando no sistema para gerar as oscilações mista e caótica. Contudo, vale salientar que nesse texto foi levantada a possibilidade do comportamento homoclínico ser obtido não só por meio dos pontos de sela-foco, mas também por meio dos pontos de sela-nó por meio do princípio de reinjeção.

Quando comparamos os resultados obtidos pela Figura 17 (a) com o diagrama de órbita calculado para $I_{F}$ versus $U$ com o valor de $\rho=180$, ver Figura 11 (a). Percebemos que os intervalos de parâmetros contendo comportamento caótico são restringidos por regiões periódicas. Essa característica associada ao fato das órbitas de Hopf se aproximarem dos pontos de sela-nó, formando assim uma órbita homoclínica fornecem as condições ideais para a formação das sequências de Farey, que segundo Koper e Gaspard [81] uma sequência de Farey deve ocorrer quando dois estados vizinhos são conectados por um encadeamento de órbitas periódicas originadas de dois estados dominantes. Outra característica levantada por Freire e Gallas [84] a respeito das sequências observadas nos modelos não-lineares, foi que alguns sistemas apresentaram uma sequência denominada de Stern-Brocot. Uma discussão mais detalhada a respeito do comportamento dinâmico abordando as sequências de Farey e Stern-Brocot exibido pelo modelo $H N-N D R$, será realizado mais a frente quando abordamos o comportamento por meio dos diagramas de períodos. 
Um ponto a ser colocado aqui e defendido nessa Tese é que o comportamento sequenciado do período por meio das regras de Farey e Stern-Brocot, deve ser observados mais facilmente em modelos que não apresentem comportamento dinâmico inseridos na condição de Shilnikov. Contudo, essa afirmação não exclui a possibilidade de observar tais fenômenos em modelos que apresentem essa condição. Entretanto, enfatizamos que as sequências de Farey e Stern-Brocot são manifestações das órbitas homoclínicas causadas pelos pontos de sela-nó e, portanto, todo sistema que apresente comportamento periódico descrito por essas regras tem que possuir duas escalas distintas de tempo, uma rápida e outra lenta na superfície estacionária (slow-fast manifold).

Para as condições de $\varepsilon=0,001$ e $\mu=50$ o sistema não apresentou uma região expressiva contendo o comportamento de Shilnikov, a região onde se observa esse comportamento fica em torno dos limites $30<\rho<100$ e $215<U<250$. Contudo, a variação dos parâmetros $\varepsilon$ e $\mu$ pode alterar tanto a forma, como o comportamento do sistema no interior da bifurcação de Hopf. As variações nas curvas de Hopf para o plano $\rho$ versus $U$, podem ser visualizadas nas Figuras 18 (a) e (c) que descrevem o comportamento das bifurcações para uma variação dos parâmetros $\varepsilon$ e $\mu$ respectivamente. Já as Figuras 18 (b) e (d), variação de $\varepsilon$ e $\mu$, correspondem às diferenças entre as intensidades normalizadas levando em consideração o máximo e mínimo da série temporal, onde as variáveis normalizadas são representadas por $\phi_{N}, c_{N}, \theta_{N} \mathrm{e}$ $I_{F N}$. Para as respectivas variações de $\varepsilon$ e $\mu$ foram usados os valores de $\rho=50, U=250, \rho=55$ e $U=250,2$ referente às Figuras 18 (b) e (d).

Estudando o comportamento apresentado nas Figuras 18 (a) e (c), referente à bifurcação de Hopf, notamos na medida em que variamos os parâmetros $\varepsilon$ e $\mu$ que a bifurcação de Hopf sofre alterações na sua morfologia. Quando observamos melhor a Figura 18 (a) percebemos que a variação do parâmetro $\varepsilon$ para valores maiores, provoca um deslocamento do comportamento $H N-N D R$ para o comportamento $N-N D R$ manifestado pelo estreitamento da bifurcação de Hopf. Entretanto quando variamos o parâmetro $\mu$, Figura 18 (c), o comportamento observado para o modelo não tem mostrado uma variação muito significativa para uma diminuição do parâmetro até o valor de $\mu=10$. Por outro lado, se passarmos desse valor notamos uma variação muito brusca da bifurcação e o comportamento apresentado passa a ser 
$N-N D R$. O comportamento $N-N D R$ apresetado pelo modelo $H N-N D R$ formado pelas Equações 22, 23 e 24 mostra que em determinadas condições o modelo pode ser utilizado para avaliar o comportamento em sistemas $N-N D R$, isso também levantar a possibilidade do modelo ser usado na descrição de sistemas que apresentem os dois comportamentos simultaneamente. Sistemas experimentais que apresentam esse comportamento simultâneo vêm sendo estudado na literatura, como exemplo temos o trabalho de Zhao et al. [85].

(a)

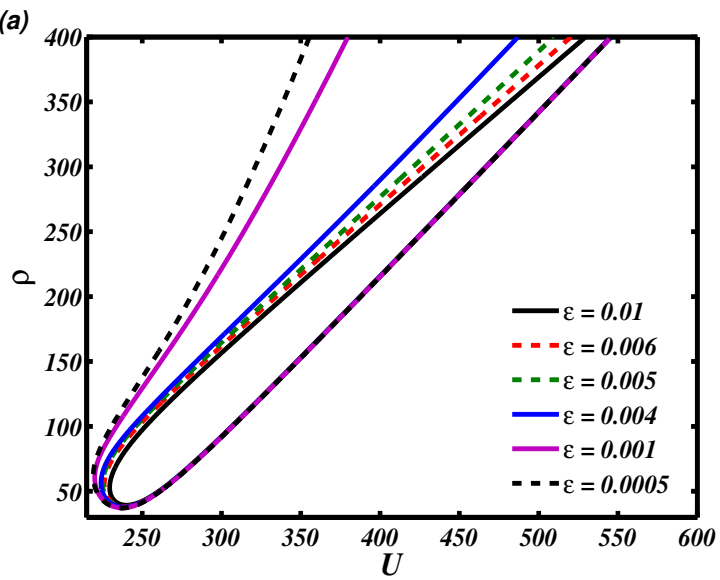

(c)

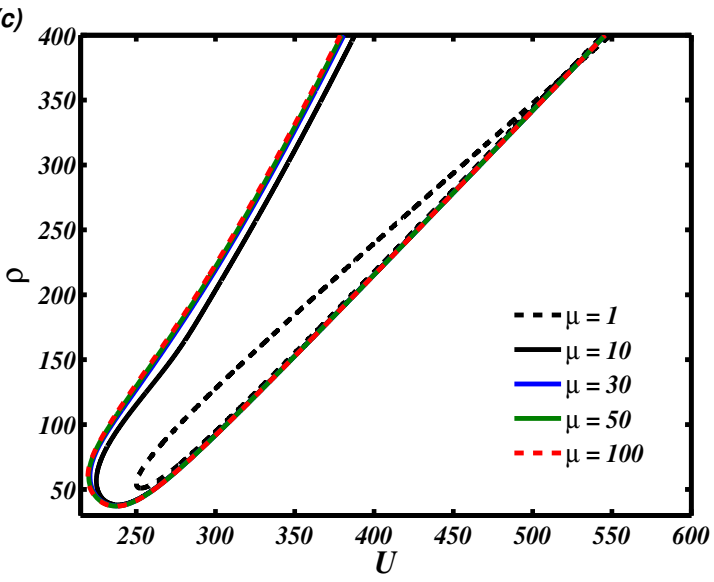

(b)

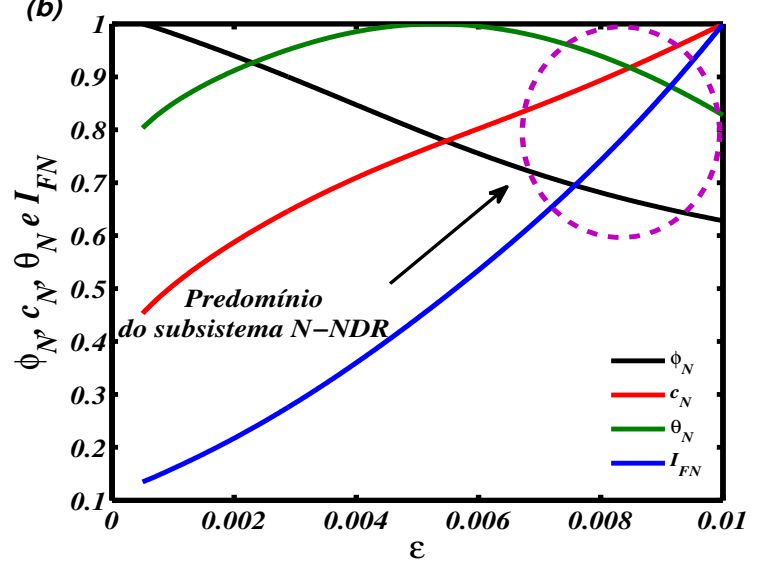

(d)

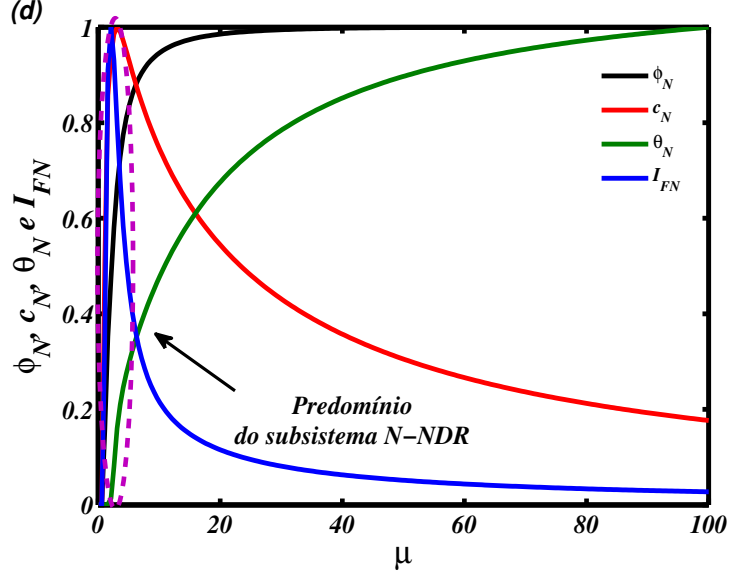

Figura 18: Diagramas de bifurcações de Hopf no espaço $\rho$ versus $U$ comparando a infuência dos parâmetros (a) $\varepsilon$ mantendo constante o valor de $\mu=50$ e (c) $\mu$ mantendo o parâmetro $\varepsilon$ constante e igual a 0,001 . Já os gráficos (b) e (d) correspondem às curvas das intensidade normalizada para $\phi_{N}, c_{N}, \theta_{N}$ e $I_{F N}$ quando variamos os parâmetros $\varepsilon$ e $\mu$ nas condições de $\rho=50, U=250$ para (b) e $\rho=55, U=250,2$ para (d).

O comportamento híbrido apresentado pelo modelo $H N-N D R$ em função dos parâmetros $\varepsilon$ e $\mu$ pode ser simplificado avaliando as Figuras 18 (b) e (d). Observe-se que na Figura 18 (b) leva em consideração a variação em $\varepsilon$. A curva referente ao comportamento normalizado das intensidades de $\theta_{N}$, linha verde, sofre uma redução em relação à curva $c_{N}$, linha vermelha, 
que aumenta sua intensidade. Essa característica faz com que os pesos dos modelos sejam alterados e o comportamento $N-N D R$ passe a dominar a dinâmica do modelo, nesse ponto o modelo começa a se comportar como um sistema $N-N D R$. Já quando olhamos para a Figura 18 (d), notamos que o comportamento das intensidades normalizadas para uma variação do parâmetro $\mu$, ocorre de forma semelhante ao da Figura 18 (b). Contudo, nota-se que para se obter o comportamento $N-N D R$ no modelo, as diferenças entre as variações nas curvas de $\theta_{N}$ e $c_{N}$ devem ultrapassar um valor crítico entorno de $\mu=10$, correspondente ao máximo da diferença entre $\theta_{N}$ e $c_{N}$. A região onde observa-se essas transições do comportamento $H N-N D R$ para $N-N D R$ e vice-versa é mostrada pelo círculo roxo tracejado nas Figuras 18 (b) e (d).

Quando variamos os parâmetros $\mu$ e, especialmente, $\varepsilon$, notamos que a forma da bifurcação muda bastante. Entretanto as Figuras 18 (a) e (c) não podem mostrar a dinâmica interna do modelo. Para solucionar esse problema, calculamos os diagramas de estabilidade para algumas variações do parâmetro $\varepsilon \operatorname{com} \mu=50$, o cálculo para $\mu$ variando não será abordado devido à variação desse parâmetro ficar praticamente inalterada para valores maiores que $\mu>10$, os gráficos para essa variação são semelhantes aos da Figura 17. Os diagramas de estabilidades para as variações de $\varepsilon=0,005,0,0005,0,004,0,006$ e 0,01 são mostrados nas Figuras 19 (a) e (b) referente ao valor de 0,005, assim com as Figuras 21 que correspondem às variações (a) 0,0005, (b) 0,004, (c) 0,006 e (d) 0,01.

Analisando a Figura 19 (a), percebe-se que o comportamento no interior da bifurcação de Hopf muda drasticamente em relação as estabilidades apresentadas pelos pontos de selafoco do tipo II. Note que as condições de Shilnikov referente a $0<v<0,5$ e $0,5<v<1$, regiões preta e cinza escuro respectivamente, dominam quase que totalmente a região de selafoco com exceção de uma pequena região nos limites de $300<\rho<400$ e $390<U<550$. Já se observarmos a Figura 19 (b) e compararmos com a Figura 17 (b), notamos que as bifurcações com respeito aos pontos de sela-nó do tipo I e II permanecem praticamente inalteradas. Apesar das características das regiões de sela-nó serem praticamente as mesmas, no que diz respeito às regiões do espaço de parâmetros, a dinâmica imposta por essa região é completamente diferente. Contudo, o princípio de reinjeção causado pelas órbitas homoclínicas de sela-nó ainda é válido, esse princípio é mostrado na Figura 20. 
(a)

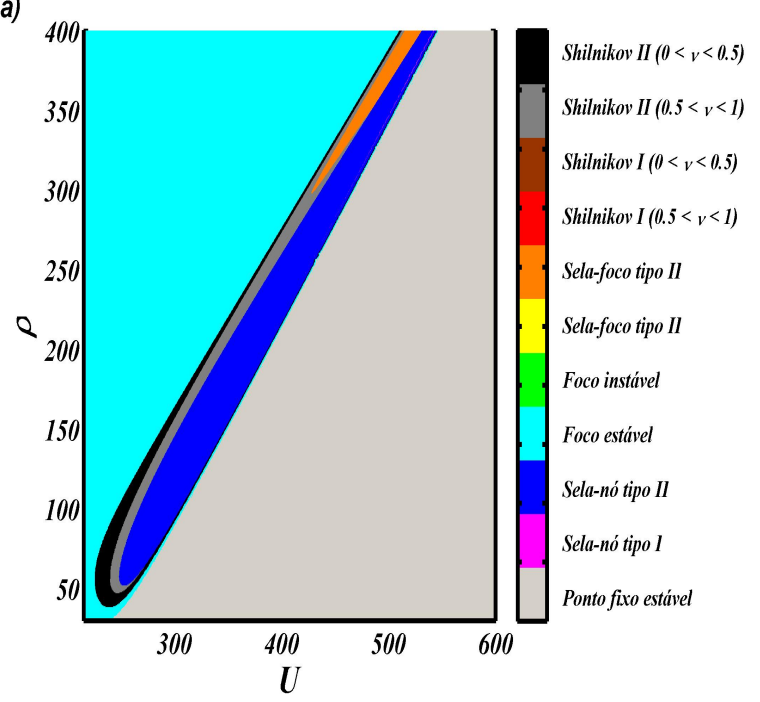

(b)

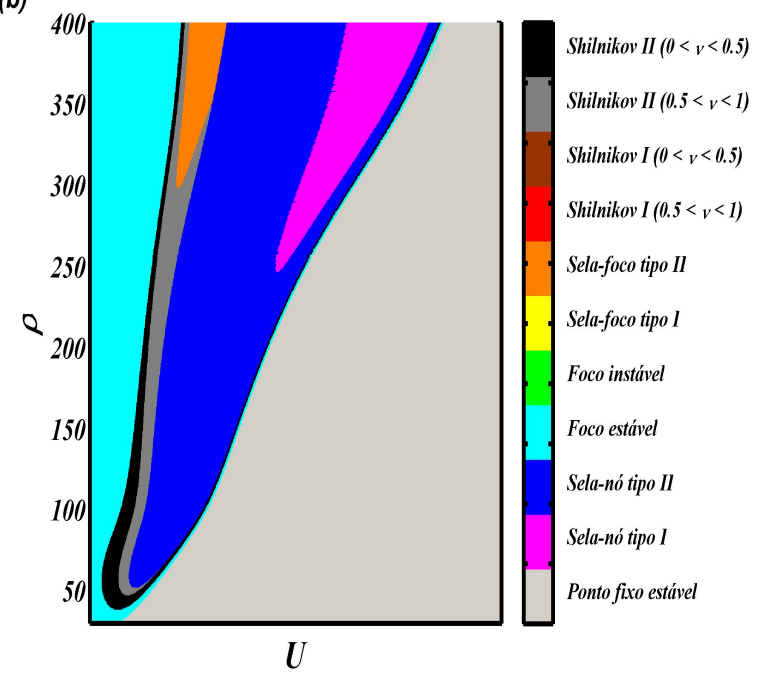

Figura 19: Estabilidades pontuais para o espaço de parâmetros $\rho$ versus $U$ calculados para o valore de $\varepsilon=0,005$ e $\mu=50$, onde (a) corresponde ao diagrama com o parâmetro $U$ corrigido e (b) sem a correção.

Para demonstrar o fenômeno de reinjeção existente no modelo observado na condição de $\varepsilon=0,005$, foram calculadas duas curvas no estado estacionário para o plano de fase $(\phi, c)$ e $(\phi, \theta)$ nas condições de $\rho=280$ e $\mu=50$. Essa condição foi escolhida para que possamos comparar os comportamentos obtidos na Figura 20 (a) com os discutidos na Figura 15 (b). As condições utilizadas no cálculo do conjunto de órbitas referentes aos pontos de sela-nó tipo I, II e Hopf com relação às condições limite na região de instabilidade localizada à direita das curvas estacionárias são mostradas na Tabela 5.

Tabela 5: Condições para construção das três órbitas mostradas nas Figuras 20 (a) e (b), as órbitas para o ponto de sela-nó I, sela-nó II e Hopf, referem-se as condições limite da região de instabilidade localizada a direita das curvas de estabilidade.

\begin{tabular}{|c|c|c|c|}
\hline \multirow{3}{*}{$\begin{array}{l}\text { Órbitas } \\
\phi \rightarrow\end{array}$} & Sela-nó I & Sela-nó II & Hopf \\
\hline & 1 & 2 & 3 \\
\hline & 222,072 & 228,440 & 231,166 \\
\hline- & 0,193 & 0,211 & 0,215 \\
\hline$\theta-$ & 0,000 & 0,000 & 0,000 \\
\hline$U \rightarrow$ & 448.065 & 449.340 & 451,025 \\
\hline
\end{tabular}

As Figuras 20 (a) e (b) descrevem o comportamento das órbitas referentes aos pontos de Hopf, sela-nó do tipo I e II localizados a direita da curva estacionária, onde a linha vermelha 
tracejada corresponde à região de instabilidade. Note que as reinjeções dos pontos fixos ocorrem no ramo estável das curvas estacionárias (a) e (b), diferente das reinjeções mostradas na Figura 15 (b) para os mesmos valores de $\rho$ e $\mu$. Uma característica importante a ser ressaltada aqui é o fato do comportamento apresentado nas Figuras 20 (a) e (b) ser similar ao comportamento demonstrado na Figura 15 (a), sendo que suas reinjeções ocorrem na região estável da curva. Esta observação leva a crer que sistemas que apresentam comportamento dentro das condições de Shilnikov podem apresentar também comportamento homoclínico de sela-nó. Tal característica é deveras interessante, pois podemos supor a existência de sistemas onde a dinâmica apresentada possa ser influenciada pela coexistência dos dois fenômenos. De fato, a coexistência de tais fenômenos foi explorada recentemente no trabalho de Andrey Shilnikov e colaboradores [86] com relação ao modelo de Rössler e será o ponto a ser discutido mais adiante quando abordarmos o modelo por meio dos expoentes de Lyapunov.
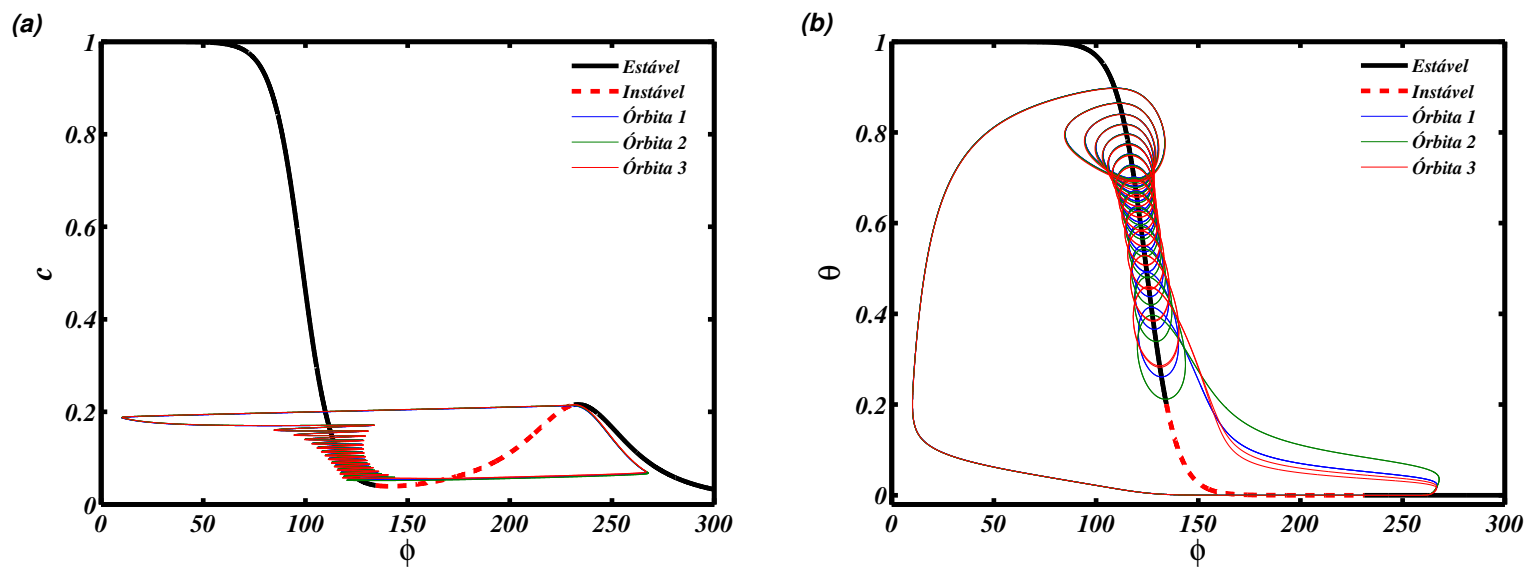

Figura 20: Órbitas nas condições de Hopf, Sela-nó I e II calculadas para o parâmetro $\rho=280$, $\varepsilon=0,005$ e $\mu=50$, onde (a) corresponde a curva estacionária no plano de fase $(\phi, c)$ e (b) referente ao plano $(\phi, \theta)$.

Uma característica peculiar apresentada pelo modelo quando variamos os parâmetros $\varepsilon$ e $\mu$, foi a mudança do comportamento dinâmico com características $H N-N D R$ para $N-N D R$. Essas mudanças não só afetam as formas das bifurcações mas também sua dinâmica interna por meio das regiões de sela-foco e sela-nó. Tal comportamento é exibido no conjunto de gráficos mostrados na Figura 21 para os valores de (a) $\varepsilon=0,0005$, (b) $\varepsilon=0,004$, (c) $\varepsilon=0,006$ e (d) $\varepsilon=0,01$ calculado para o valor de $\mu=50$.

Observando as Figuras 21 (a), (b), (c) e (d), nota-se um comportamento interessante 
em relação as regiões dentro das condições de Shilnikov quando o parâmetro $\varepsilon$ é variado. À medida em que variamos o parâmetro para valores maiores de $\varepsilon$, que corresponde a mudar a dinâmica do sistema $H N-N D R$ para $N-N D R$, as regiões de Shilnikov aumentam até dominarem toda a região de sela-foco. Esse ponto é muito importante pois indica que os fenômenos apresentados pelos sistemas nas condições de $N-N D R$ possuem uma forte influência dos pontos de sela-foco proporcionando o cenário adequado para a formação de estruturas espirais auto-organizadas conhecidas como cascatas caóticas. Tais estruturas serão abordados mas a frente quando falarmos dos expoentes de Lyapunov.

(a)

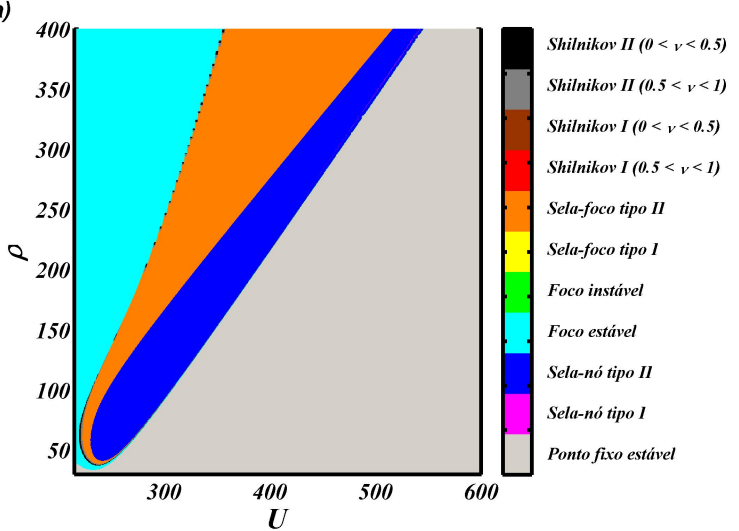

(c)

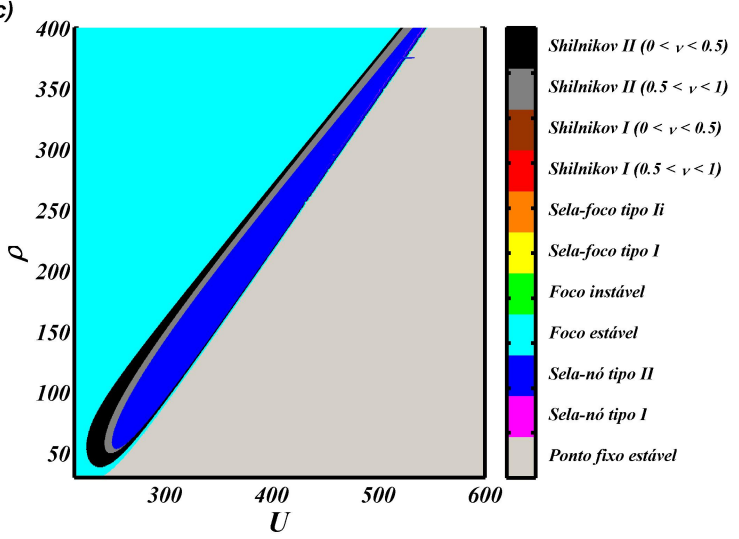

(b)

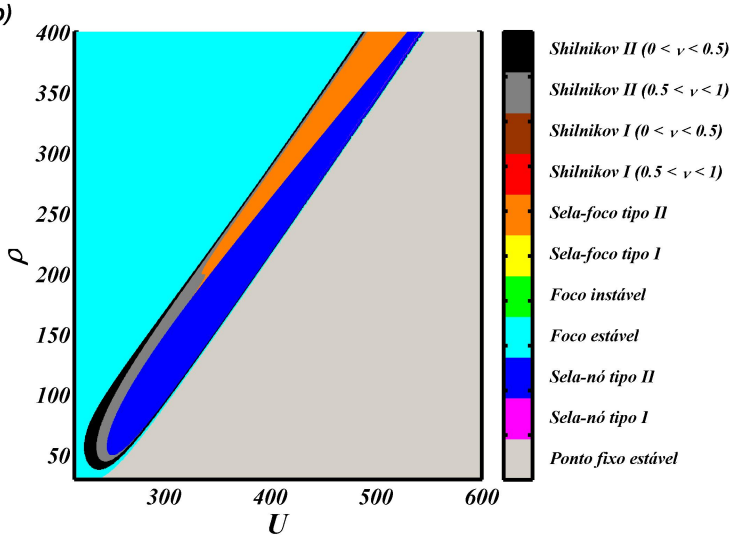

(d)

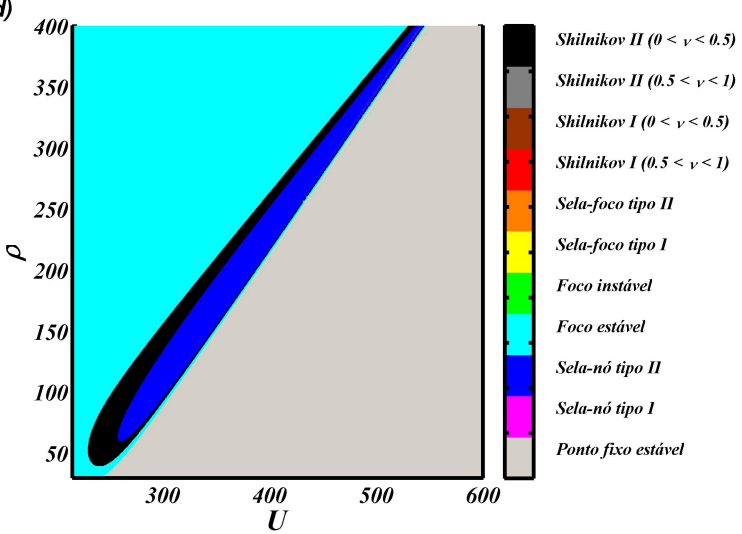

Figura 21: Diagramas de estabilidades pontuais no espaço de parâmetros $\rho$ versus $U$ com uma variação de $\varepsilon$ dada por (a) 0,0005 , (b) 0,004 , (c) 0,006 e (d) 0,01 , mantendo $\mu=50$.

Apesar dos gráficos mostrados nas Figuras 17, 19 e 21 mostrarem todas as regiões discriminado suas estabilidades em relação ao comportamento apresentado pelos pontos fixos no estado estacionário, o método ainda não é suficiente para descrever e caracterizar a dinâmica interna das bifurcações com relação ao comportamento caótico, assim como a distribuição do período ao logo da variação no plano de parâmetros dados por $\rho$ versus $U$. Para resolver essa 
dificuldade o problema foi abordado usando os diagramas de Lyapunov e de períodos.

\section{I.4.2 Diagramas de Lyapunov}

Inúmeras provas convincentes da existência de caos determinístico obtidos para diversos sistemas dissipativos não-lineares e experimentais vem sendo relatado na literatura $[87,88]$ e, portanto, a questão da determinação e quantificação de caos tornou-se um ponto importante no estudo de tais sistemas. Para realizar o diagnóstico do comportamento dinâmico nos dados experimentais e numéricos, diversos autores vêm utilizando uma ferramenta matemática chamada de expoente de Lyapunov [55], que tem como característica medir a taxa média exponencial da divergência ou convergência das órbitas no espaço de fase. As órbitas próximas correspondem aos estados idênticos indicando que o sistema não sofre uma variação acentuada com relação às condições iniciais, por outro lado, a divergência exponencial das órbitas de um sistema significa que ele se encontra em um estado completamente diferente do inicial, quando isso acontece, pode-se dizer que há uma diminuição da possibilidade de predizer o comportamento futuro do sistema. Outra características importante é que sistemas com pelo menos um expoente de Lyapunov positivo são definidos como sendo caóticos, onde a magnitude do expoente reflete a escala de tempo em que a dinâmica do sistema se torna imprevisível [89].

Um ponto interessante a respeito dos expoentes de Lyapunov é o fato de sua utilização não ser restrita somente à caracterização de caos numa análise unidimensional, mas também, podemos utilizá-lo para avaliar o comportamento dinâmico num espaço de parâmetros bidimensional revelando assim fenômenos de codimensão 2. Fenômenos de codimensão 2 vem sendo intensivamente estudado por diversos pesquisadores como exemplo podemos citar os trabalhos de Gallas et al. [90, 91] para o modelo do Laser de $\mathrm{CO}_{2}$.

Nesse ponto da Tese iremos descrever a dinâmica interna do modelo com base nos dados obtidos pelo expoentes de Lyapunov e fazer uma comparação com os dados referente aos diagramas de estabilidade pontual. Para isso foi calculado os diagramas de Lyapunivo no plano de parâmetros $\rho$ versus $U$ com as condições de $\varepsilon=0,001$ e $\mu=50$. um ponto importante aqui, é que em todos os diagramas de Lyapunov calculados foi mantido o parâmetro $\mu$ sempre 
constante. O motivo para isso é que a bifurcação de Hopf não sofre uma alteração na sua forma para valores de $\mu>10$, ver Figura 18 (c). A distribuição dos expoentes de Lyapunov para o sistema em questão pode ser visualizada na Figura 22.

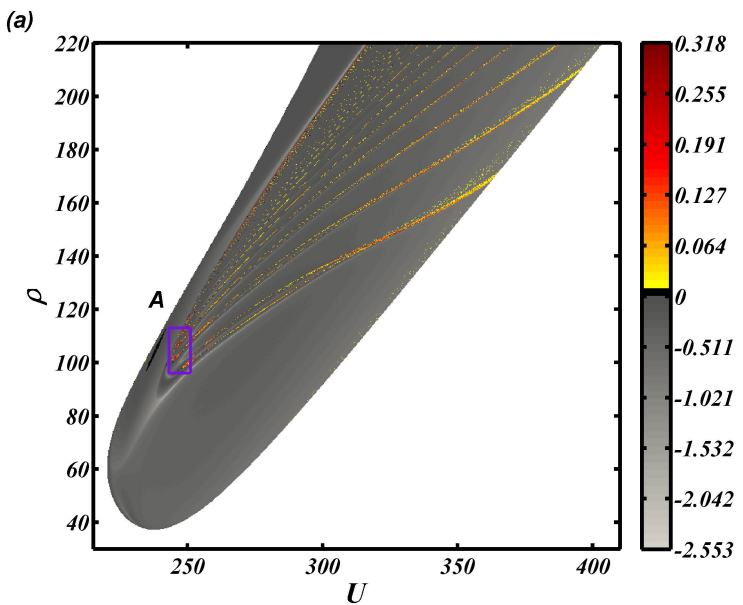

(b)

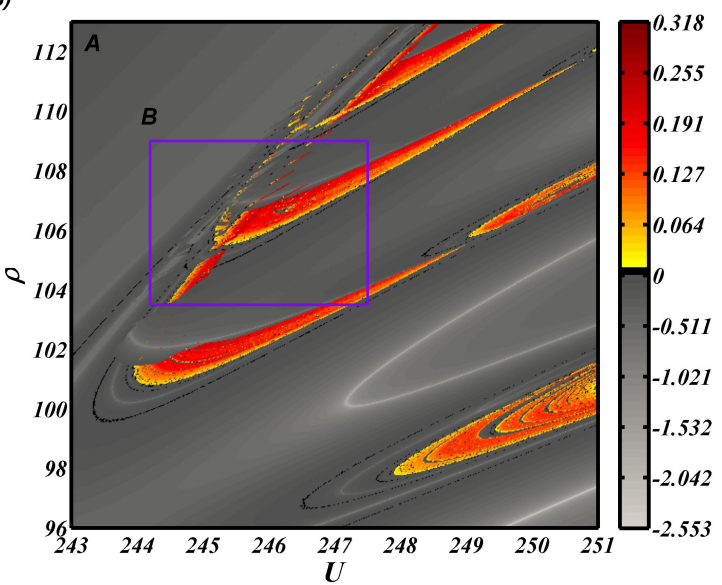

(d)

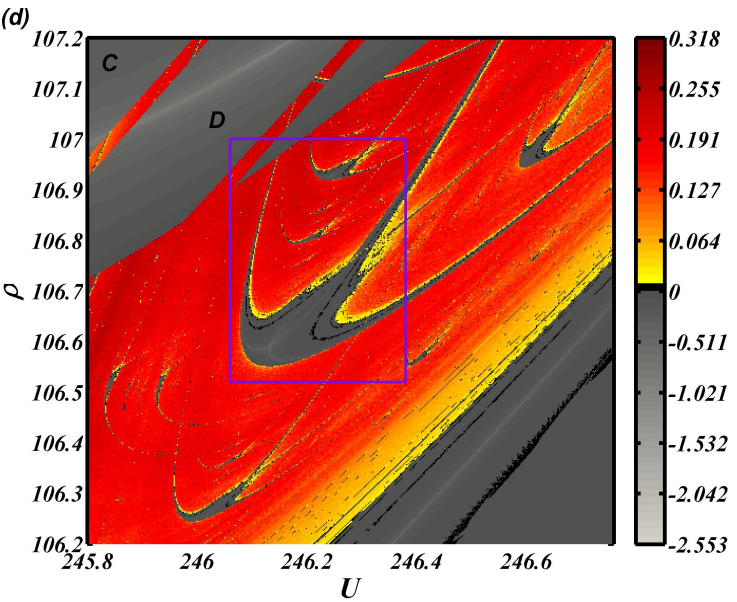

(c)

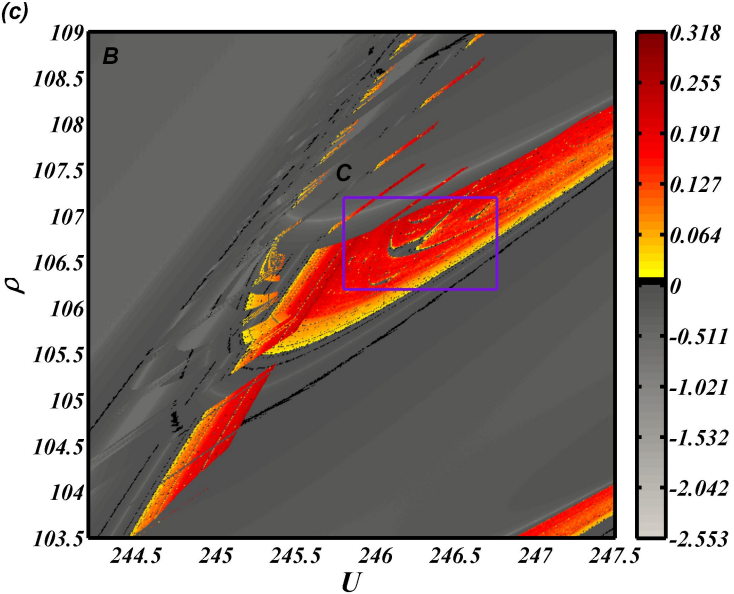

(e)

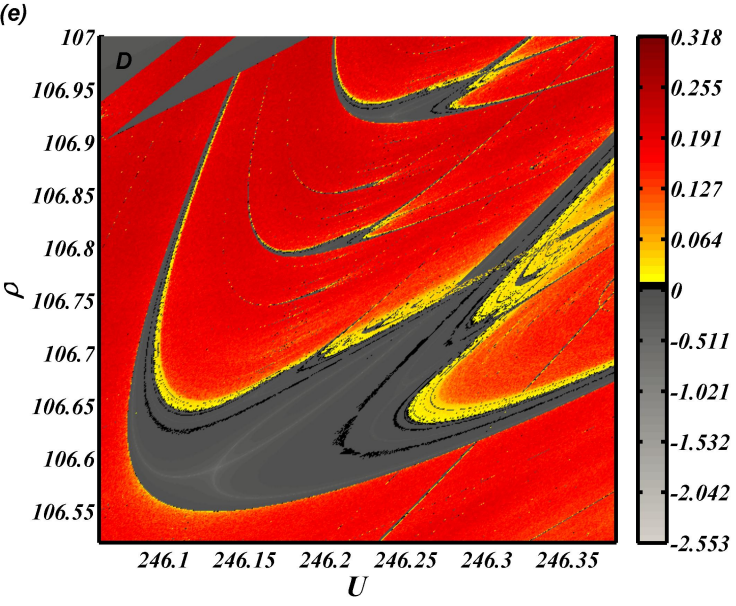

Figura 22: Diagramas de Lyapunov calculados para $\varepsilon=0,001$ e $\mu=50$ no plano de parâmetro $\rho$ versus $U$, onde a Figura 22a corresponde a uma resolução de $700 \times 700$, já os gráficos (b), (c), (d) e (e) corresponde a uma resolução de 500x500. 
A Figura 22 mostra os diagramas de Lyapunov para o sistema $H N-N D R$ em modo potenciostático com os expoentes positivos representados na escala de cor vermelha e os expoentes negativos na escala cinza. Observando a Figura 22 (a) nota-se a presença de linhas caóticas percorrendo toda a extensão do diagrama e convergindo para uma região localizada dentro do retângulo roxo $\mathrm{A}$, onde é possível distinguir uma estrutura muito rica de domínios caóticos. Já as Figuras 22 (b), (c), (d) e (e) são ampliações das regiões dentro dos retângulos roxos A, B, C e D respectivamente. Vale salientar ainda que uma série alternada e concêntrica de regiões caóticas e periódicas é claramente observada em todo o canto inferior direito da Figura 22 (b). Tanto a distribuição geral apresentada na Figura 22 quanto às estruturas concêntricas lembram os resultados numéricos obtidos recentemente para a reação de Belousov-Zhabotinsky [91]. Além de regiões com fronteiras bem definidas, Figura 22 (c). Essa figura mostra também que o domínio caótico apresenta interrupções abruptas, ou seja, não variam suavemente com os parâmetros. Na verdade, tais descontinuidades são apenas aparentes, indicando a presença de multiestabilidade no sistema.

Na medida em que analisamos as ampliações nas regiões, nota-se o surgimento de padrões emergentes de diferentes tamanhos, como o que pode ser observado nas Figuras 22 (c) e (d). Tais padrões são comumente conhecidos na literatura como camarões ou shrimps $[92,93]$. A presença desses objetos reforça o caráter universal da sua ocorrência, conforme relatado anteriormente para uma ampla variedade de modelos de tempo contínuo e discreto [94]. Já a Figura 22 (e) mostra uma visão detalhada do shrimp selecionado em 22 (d). Observando detalhadamente 22 (e), nota-se a existência de linhas brancas no interior das ilhas periódicas. Essas linhas fazem referência aos expoentes mais negativos que cruzam o domínio do shrimp de forma organizada e aparentemente simétrica.

Outro ponto a ser discutido com relação aos expoentes de Lyapunov é a respeito das linhas pretas nos gráficos, ver Figura 22 (d) e (e). Essas linhas fazem referência ao valor zero assumido pelos expoentes. $\mathrm{O}$ expoente zero indica um ponto de extrema instabilidade e pode ser associado ao um ponto de bifurcação. Logo, pode-se notar em 22 (e) a existência de bifurcações dentro das ilhas periódicas, shrimps, indicando a existência de uma rota para o caos no interior das ilhas. 
A Figura 19 mostra um diagrama de Lyapunov para uma variação do parâmetro $\varepsilon=$ 0,005 , onde os retangulos azul e verde correspondem as ampliações das regiões $A$ e $B$ respectivamente.

(a)

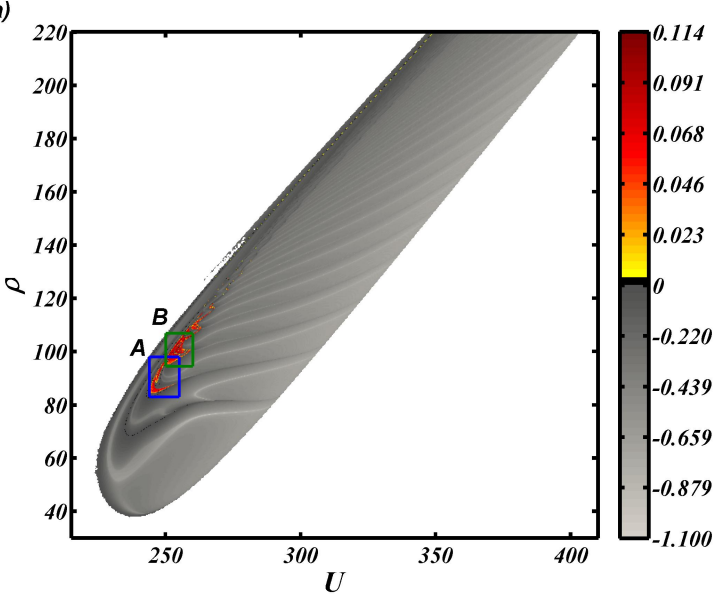

(c)

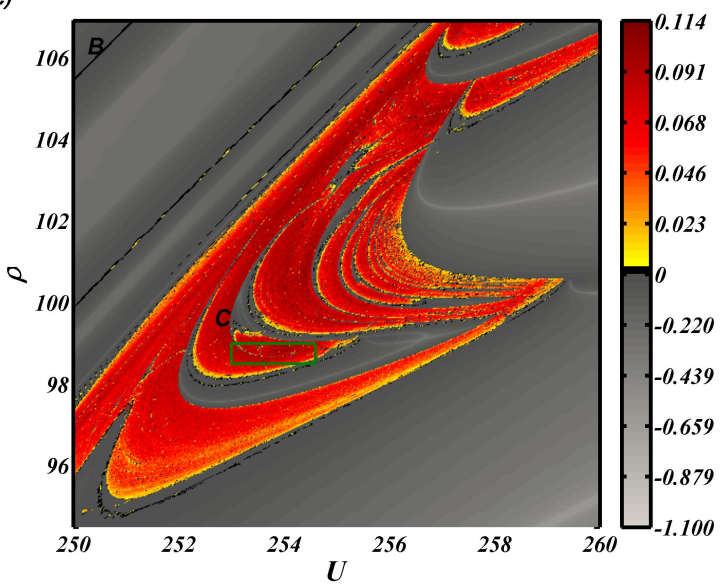

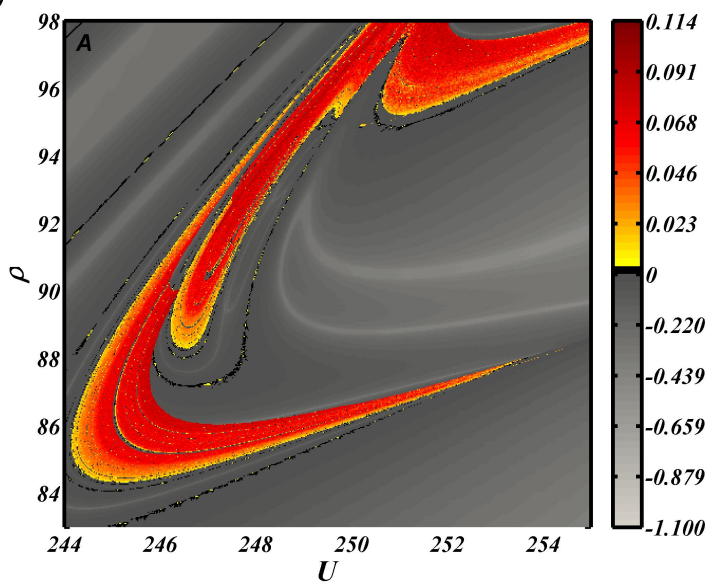

(d)

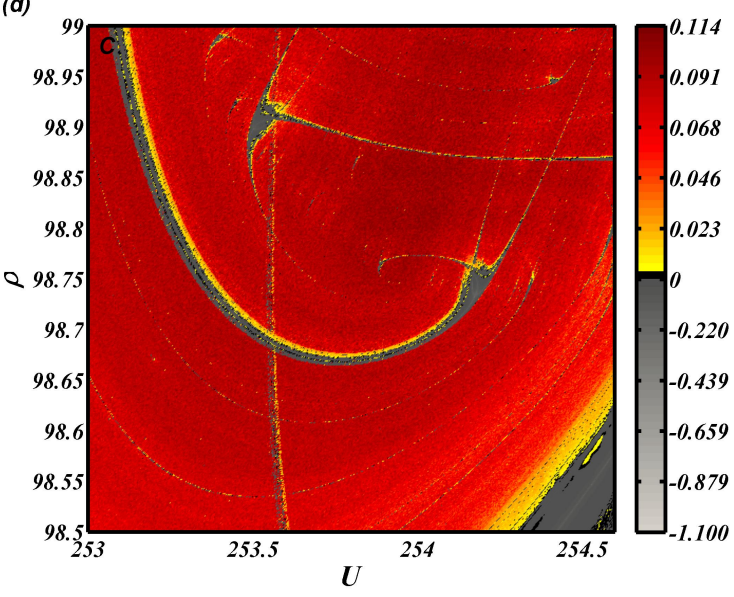

Figura 23: Diagramas de Lyapunov para $\varepsilon=0,005$ e $\mu=50$ no plano $\rho$ versus $U$, onde o gráfico (a) possui uma resolução de $700 \times 700$. Os gráficos (b) e (c) correspondem as áreas A e $\mathrm{B}$, já o gráfico (d) corresponde a área $\mathrm{C}$ do gráfico (c), todos com resolução de 500x500.

Quando variamos o parâmetro $\varepsilon$ para o valor de 0,005 , ver Figura 23 (a), nota-se que a dinâmica interna da bifurcação muda completamente em relação à Figura 22 (a). Foi observado na Figura 19 (a), referentes ao diagrama de estabilidade, que a variação do parâmetro $\varepsilon$, apresentou um comportamento dinâmico dentro das condições de Shilnikov referentes aos pontos de sela-foco, diferentemente do comportamento apresentado pelos pontos de sela-foco na Figura 17 (a). Comparando as Figuras 23 (a) e 22 (a), percebe-se que o comportamento caótico na Figura 23 (a) é mais localizado, ao contrário da Figura 22 (a) que apresentou linhas caóticas percorrendo toda a região de bifurcação. Tais observações mostram que o sistema apresen- 
tando comportamento dinâmico fora da condição de Shilnikov deve apresentar um fluxo mais disperso. Tal dispersão do comportamento pode ser causada pelo comportamento homoclínico presente nos pontos de sela-nó.

Fazendo as ampliações nas regiões $A$ e $B$ referente as Figuras 23 (b) e (c), observase que as regiões caóticas estão organizada na forma de dobras que por sua fez reorganizam o comportamento periódico. Esse tipo de organização leva a formação das estruturas chamadas de hubs [95], ver Figura 23 (d), que corresponde a um conjunto de funções periódicas conectadas entre si, tais estruturas foram abordadas por Gaspard e colaboradores [72, 73] por meio das órbitas homoclínicas de Shilnikov. Entretanto, a formação desses hubs não está restrita só aos sistemas que apresentam o comportamento de Shilnikov, mais sim a uma combinação das dinâmicas homoclínics causadas pelos pontos sela-nó e sela-foco [86]. De fato, sistemas apresentando huds fora das condições de Shilnikov foram relatados por Freire e Gallas [96] para o modelo do laser semicondutor. Essa característica importante relacionada à formação das estruturas no interior das bifurcação, não só para o modelo aqui estudado, mas sim, para todos os modelos que de certa forma apresentam comportamento caótico auto-organizado na forma de hubs e shrimps, ver Figuras (d) e (e), é o fato de tais comportamentos estarem associados às órbitas homoclínicas apresentadas nesses modelos e suas superfícies estacionária terem a forma de $S$. Logo pode-se afirmar que quando as condições não satisfazem o critério de Shilnikov, mas possuem um comportamento dinâmico homoclínico de sela-nó, o sistema pode apresentar uma dinâmica equivalente ao demonstrado na Figura 22 (a).

Para verificar as influência do comportamento de Shilnikov na dinâmica apresentada pelo sistema por meio dos expoentes de Lyapunov. Foi calculado um conjunto de gráficos para os valores de $\varepsilon=0,0005,0,004,0,006$ e 0,01 mostrados nas Figuras 24 (a), (b), (c) e (d) respectivamente. 
(a)

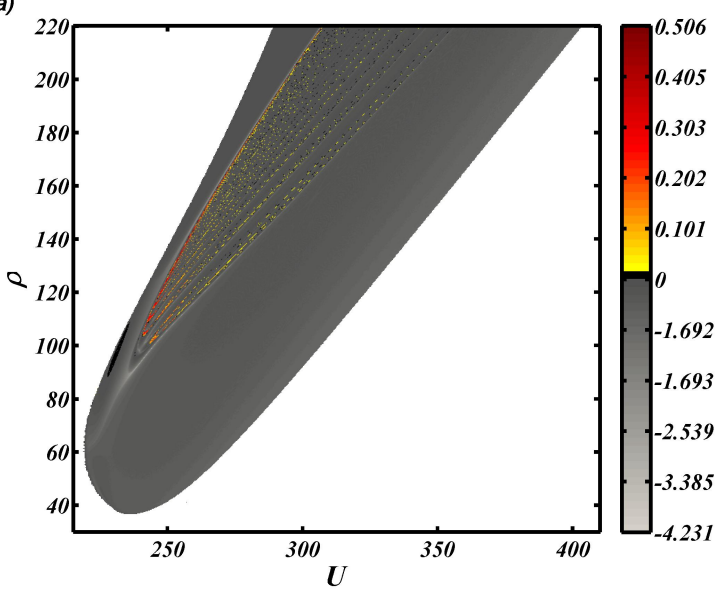

(c)

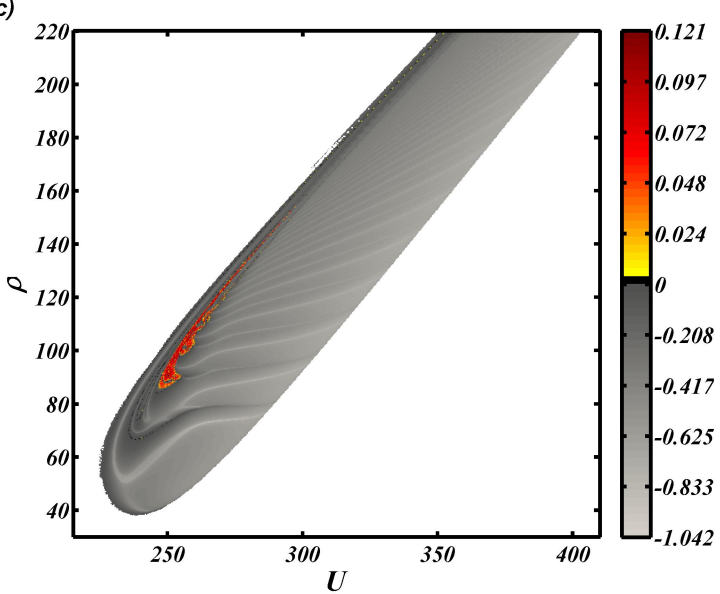

(b)

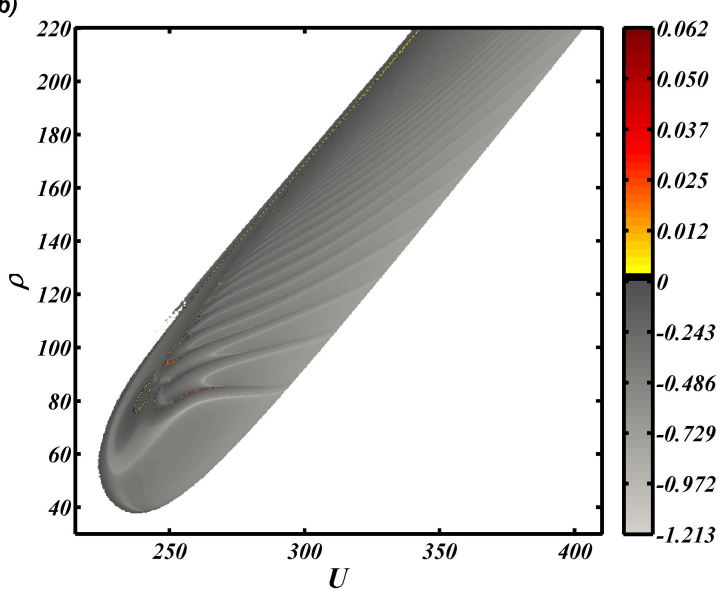

(d)

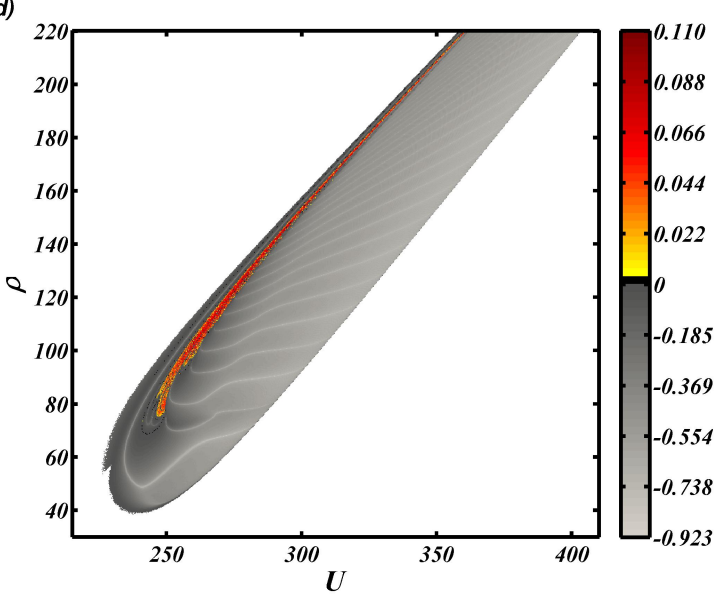

Figura 24: Diagramas de Lyapunov com resolução de $700 \times 700$ no espaço de parâmetros $\rho$ versus $U$ para o sistema $H N-N D R$ em modo potenciostatico com o parâmetro $\varepsilon$ variando de (a) 0,0005 , (b) 0,004 , (c) 0,006 e (d) 0,01 mantendo o parâmetro $\mu=50$.

Observando as Figuras 24 (a), (b), (c) e (d), nota-se que as regiões caóticas tendem a se concentrarem no lado esquerdo da bifurcação de Hopf à medida que o parâmetro $\varepsilon$ aumenta. Quando comparamos esses resultados com os obtidos para os diagramas de estabilidade mostrados nas Figuras 21 (a), (b), (c) e (d), observa-se uma relação muito forte em relação à localização das regiões caóticas com a condição de Shilnikov. Repare que para a condição da Figura 24 (a) correspondente ao cenário não shinikoviano, temos que a dispersão das regiões caóticas forma linhas muito semelhante às observadas na Figura 22 (a), esse comportamento pode ser atribuído ao cenário homoclínico de sela-nó existente no modelo. Tal comportamento também foi observado no modelo químico para a reação de Belousov-Zhabotinsky [91, 97] e para os modelos de Bonhoeffer-van der Pol e FitzHugh-Nagumo [84]. 


\section{I.4.3 Diagramas de Período}

A Figura 25, mostra os diagramas de período para o sistema nas condições de $\varepsilon=$ 0,001 e $\mu=50$, onde as regiões caóticas são representadas em branco no interior da bifurcação de Hopf e os períodos das regiões são mostrados no mapa de cor ao lado da figura. Os retângulos em preto correspondem às regiões ampliadas e a linha vermelha tracejada em 25 (a), refere-se a condição do diagrama de órbita calculado para $U=280$ na Figura 26.
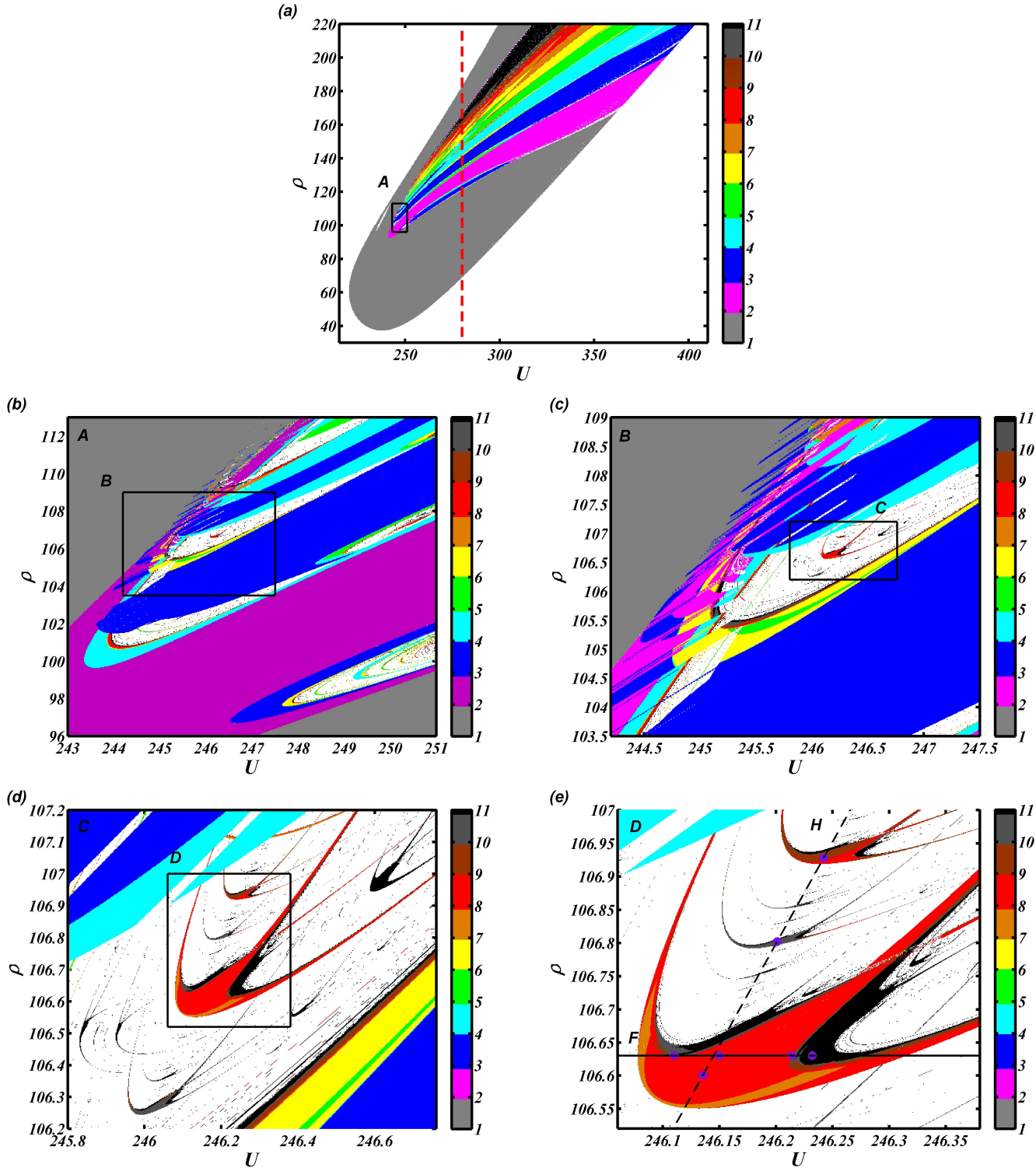

Figura 25: Diagramas de período obtidos para o espaço de parâmetros $\rho$ versus $U$ com $\varepsilon=$ 0,001 e $\mu=50$, onde a escala de cor indica o período entre 1 e 11 . Os períodos acima de 11 estão indicados pela cor preta. A Figura 25 (a) corresponde a uma resolução de $700 x 700$, já os gráficos (b), (c), (d), e (e) possuem uma resolução de 500x500. 
Na Figura 25 (a), pode-se observar claramente a organização estrutural dos períodos, onde tem-se uma grande região em cinza claro correspondente as oscilações de período 1. Quando aumentamos o valor do parâmetro $\rho$ percebe-se que o sistema sofre sucessivas bifurcação duplicando seu período. Note também que o período 2 indicado pela cor roxa, desenvolve dois ramos periódicos que formam uma cascata de duplicação periódica. Um ponto interessante a ser discutido aqui é a formação praticamente "simétrica" do comportamento dinâmico quando observado no espaço de parâmetros $\rho$ versus $U$. Tal simetria em relação ao fenômeno de duplicação pode ser correlacionada com conjuntos ordenados de números racionais. Para isso, primeiramente iremos estabelecer uma nomenclatura para representar o comportamento oscilatório no interior da bifurcação obtida para o modelo $H N-N D R$, que nesse caso foi $l^{p}$, onde $l$ representa os números de oscilações com alta amplitude e o termo do expoente $p$ representa os números de oscilações com baixa amplitude observadas durante a evolução no tempo, a soma dos termos de baixa e alta amplitude informa o período da série temporal.

Quando observamos a distribuição do comportamento oscilatório para os diagramas de órbitas da Figura 26, correspondente a linha em vermelho tracejado na Figura 25 (a) calculada para uma variação do parâmetro $\rho$ mantendo $u=280$. Observa-se que existe um conjunto de bifurcações com comportamento periódico bem definido que pode ser divido em duas sequências, em que a primeira, indicada pelas linhas azuis na Figura 26 (b), correspondente a uma ampliação da região roxa na Figura 26 (a), será chamada de $A$ e representa o conjunto formados pelas oscilações $1^{p}$ para $p=1,2,3, \ldots, n$. Já a segunda sequência, indicada pela linha vermelha na Figura 26 (b), será chamada de sequência $B$ representando o conjunto formado por $2^{p}$ para $p=1,2,3, \ldots, n$.

Uma característica intrigante quando avaliamos os períodos no modelo usando a notação $l^{p}$, é que podemos representar seu comportamento utilizando uma sequência de números racionais. Em trabalhos realizados por Freire e Gallas $[84,97]$ foi relatado que o comportamento períodico para alguns sistemas dinâmicos podem ser representados por meio da sequência numérica de Stern-Brocot. Também podemos citar o trabalho de Koper e Gaspard referente a modelagem de sistemas eletroquímicos [81], nesse trabalho eles enfatizam que o comportamento no interior a bifurcação de Hopf segue uma sequência numérica de Farey. Entretanto, 
quando comparamos as duas sequências percebe-se que a sequência de Stern-Brocot é mais geral que a sequência de Farey. Para mostrar essa diferença foram construídos dois esquemas mostrando a distribuição dos ramos para as árvores de Farey, ver Figura 27 (a), e Stern-Brocot Figura 27 (b).

(a)

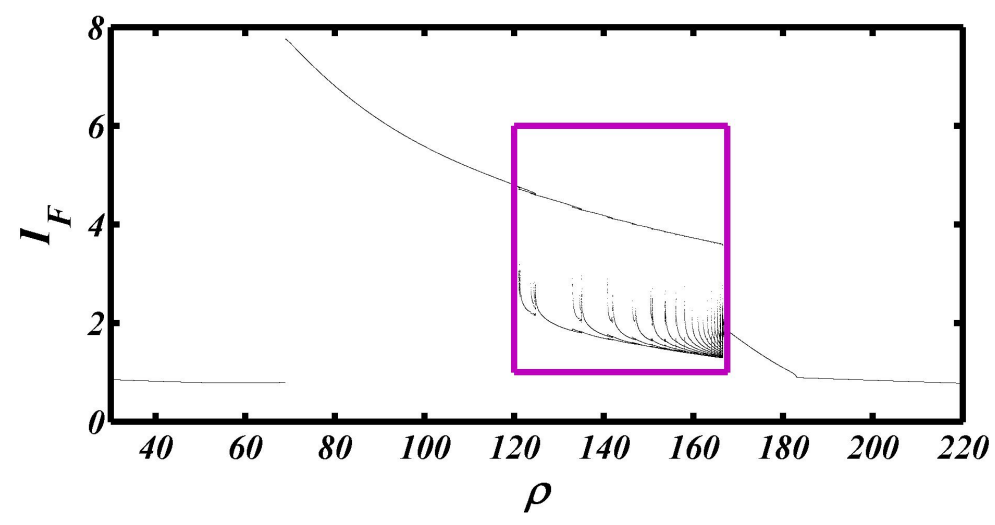

(b)

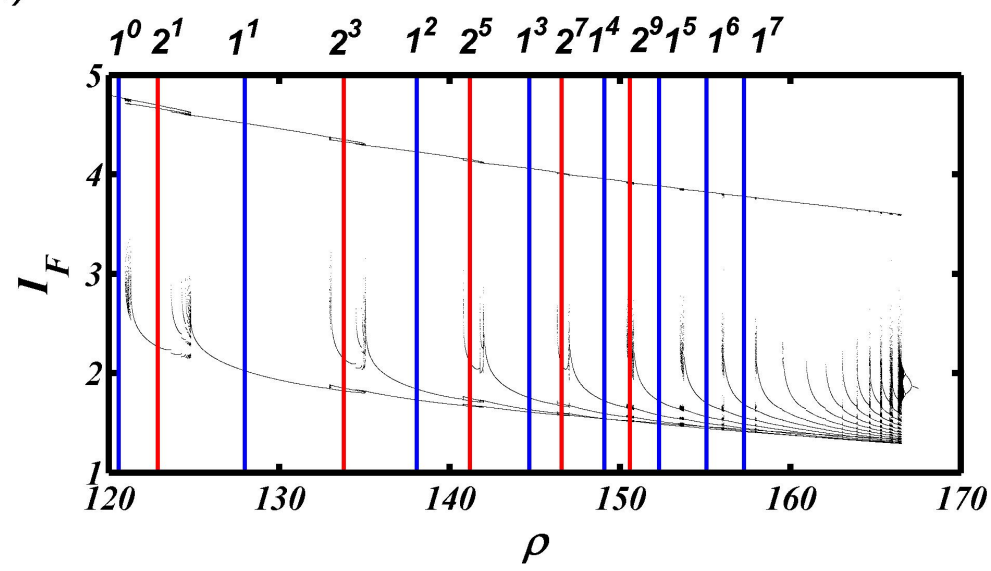

Figura 26: Diagrama de órbitas para a linha vermelha tracejada indicada na Figura 25 (a) referente ao valor de $U=280$ com $\rho$ variando. O gráfico (b) corresponde a uma ampliação da região interna ao retângulo roxo em (a). As linhas azuis e vermelhas representam as sequências $A$ e $B$ respectivamente.

Ao comparar as sequências dos ramos, tanto para a árvore de Farey como para SternBrocot Figura 27 (a) e (b) respectivamente, com a Figura 26 (b) correspondente ao diagrama de órbitas para a linha vermelha tracejada na Figura 25 (a). Tem-se que a sequência apresentada durante a variação de $\rho$ correspondem aos ramos destacados em azul e vermelho correspondente às sequências $A$ e $B$. Note que o comportamento na árvore de Farey explica de forma incompleta a sequência observada na Figura 26 (b), pois não especifica a formação do período $1^{1}$, $\operatorname{logo}$ 
temos que os domínios iniciais para se obter a sequência são $1^{0}$ e $1^{1}$. Isso indica que no diagrama de período mostrado na Figura 25 (a) teríamos que ter uma região assimétrica separadas por dois comportamentos diferentes, um referente ao período $1^{0}$ e o outro referente a $1^{1}$. Em contra partida quando avaliamos a sequência obtida pela árvore de Stern-Brocot, percebe-se que os domínios iniciais para se obter a sequência são $1^{0}$ e $1^{0}$. Tal característica é confirmada quando se observa o diagrama de período na Figura 25 (a), onde temos uma simetria na distribuição do período. Portanto, pode-se afirmar que para os valores de parâmetros $\varepsilon=0,001$ e $\mu=50$ a dinâmica apresentada para o modelo é compatível com a descrição das sequências de SternBrocot.

Um ponto importante a ser mencionado aqui é que as condições exibidas pelo diagrama de período na Figura 25 (a) correspondem às condições fora do critério de Shilnikov. Esse é um ponto muito interessante pois pode indicar que sequências descrita pela árvore de Stern-Brocot podem ser mas facilmente observadas. Em contrapartida, quando temos condições de Shilnikov as sequências devem ser aproximadas pela árvore de Farey. O motivo é que nas condições em que o sistema apresentou comportamento de Shilnikov dominante o modelo perdeu "simetria" nas regiões caóticas devido ao acúmulo dos períodos se intensificarem nas regiões de Shilnikov.

(a)

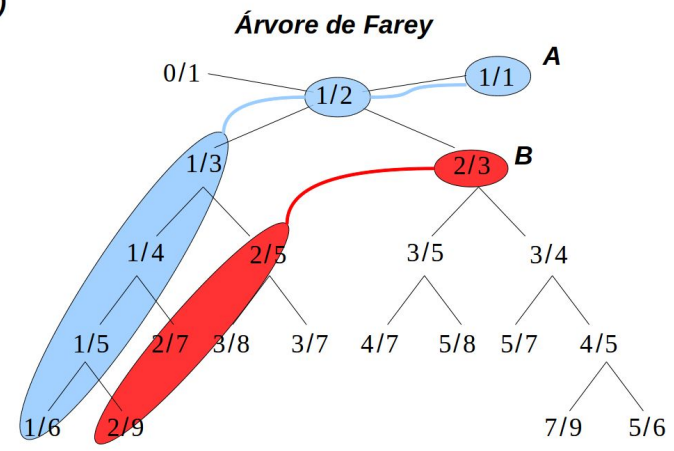

(b)

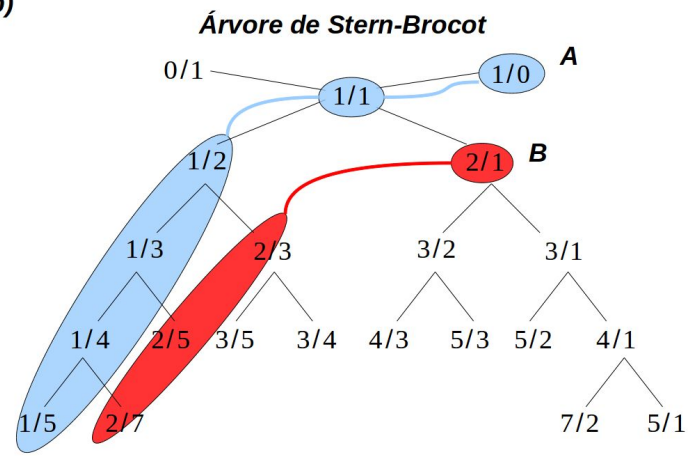

Figura 27: Esquema mostrando a distribuição dos período para a árvore de (a) Farey e (b) Stern-Brocot.

Quando se avalia o comportamento oscilatório no interior das estruturas periódicas denominadas de shrimps Figuras 25 (b), (c), (d) e (e), nota-se a transição por meio de duplicação do período indo na direção das regiões caóticas. Contudo, quando comparamos os períodos entre as estruturas, ver Figura 25 (e), percebe-se que não é possível determinar, a princípio, 
uma rota para o caos. Para auxiliar na descrição do comportamento interno dos shrimps foram calculados dois diagramas de órbitas referentes às linhas $F$ e $H$ em preto que cortam a Figura 25 (e), onde a linha contínua $F$ representa o diagrama de órbitas calculado para o valor de $\rho=106,63$ com $U$ variando. Já a linha tracejada $H$ refere-se ao diagrama de órbitas obtido para uma variação de $U$ e $\rho$ dada pela Equação 39, os diagramas de órbitas são mostrados nas Figuras 28 (a) e (b).

$$
U=211,36+0,33 \rho
$$

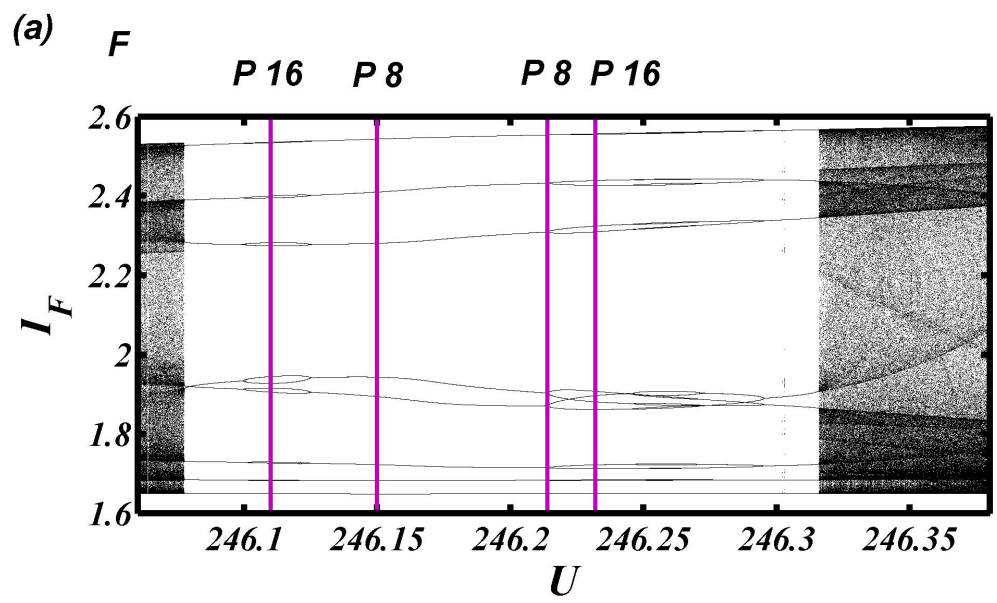

(b) $H$

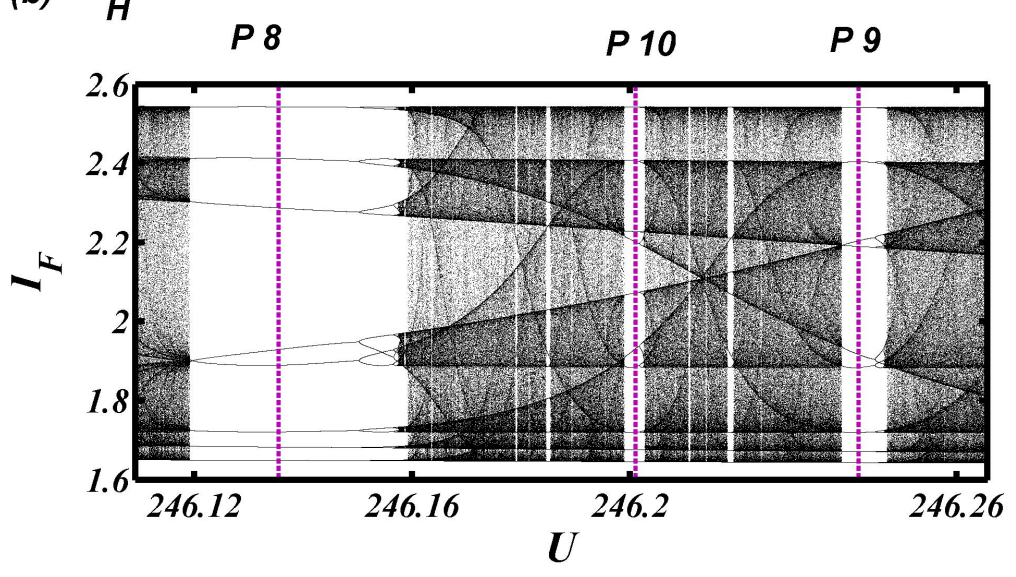

Figura 28: Diagramas de órbitas para as condições referente às linhas (a) $F$ calculada para $\rho=$ 106,63 e (b) $H$ correspondente variação de $U$ e $\rho$ dada pela Equação 39. As linhas continuas e tracejadas nas figuras indicam o período referentes aos pontos roxos na Figura 25 (e).

Observando os diagramas de órbitas para as Figuras 28 (a) e (b), referentes à Figura 25 (e). Nota-se que o comportamento apresentado no interior das estruturas, shrimps, sofre um conjunto de bifurcações do tipo duplicação de período indicando pela linha roxa contínua 
e tracejada nas Figuras 28 (a) e (b) respectivamente. Contudo, quando avaliamos as estruturas entre os vários shrimps, Figura 28 (b) referente a reta $H$ na Figura 25 (e). Percebe-se que a rota do comportamento dinâmico não é tão evidente em relação ao comportamento apresentado no interior de cada shrimp. Outro ponto interessante é a existência de uma rede formada por bifurcações de duplicação de período no interior das estruturas. Essa característica nos remete ao Teorema de Gavrilov e Shilnikov [74] que estabelece que para cada bifurcação de duplicação de período temos uma bifurcação de sela-nó associada. De fato Bonatto [50] mostrou esse comportamento para o modelo do circuito eletrônico simétrico. 


\section{I.5 Conclusões}

Apresentou-se nesta primeira parte da Tese um estudo numérico sistemático de um modelo genérico para um oscilador eletroquímico. A análise foi realizada por meio de uma investigação aprofundada dos diagramas de bifurcação e diagramas de fase de alta resolução levando em consideração dois parâmetros relevantes em condições experimentais. Esta é a primeira análise deste tipo para um sistema eletroquímico, representado por um modelo mínimo de 3 equações diferenciais autônomas.

Os resultados obtidos com relação ao comportamento apresentados pelos diagramas de estabilidades pontuais revelou a existência do cenário homoclínico pelos pondo de sela-foco, referentes à condição de Shilnikov. Também foi mostrado que nas condições de parâmetro em que não se observa um cenário de Shilnikov a dinâmica observada é causada pelos pontos de sela-nó por meio do princípio de reinjeção demonstrando a existência de duas regiões com escalas de tempo bem definidas (slow-fast manifold).

Quando avaliamos a dinâmica interna da bifurcação de Hopf por meio dos expoentes de Lyapunov observou-se a existência de estruturas complexas de domínios periódicos incorporados em um fundo caótico. Os shrimps observados em outros sistemas distintos também foram detectados no sistema estudado aqui. Esse resultado enfatiza o caráter universal da ocorrência de tais estruturas. Contudo, pode-se observar que tais estruturas estão relacionadas com o comportamento homoclínico nos sistemas e que é retratado nessa tese.

Outro ponto fundamental estudado aqui, foi a descrição da sequência periódica no interior da bifurcação que pode ser explicada utilizando as árvores de Farey e Stern-Brocot, onde foi observado que para a condição de $\varepsilon=0,001$ o comportamento interno pode ser descrito pela sequência de Stern-Brocot. 


\section{Parte II}

\section{Análise Multivariada Aplicada a Séries Temporais Experimentais}




\section{II.1 Introdução}

Como já mencionado, várias reações de eletro-oxidação de moléculas orgânicas pequenas podem apresentar instabilidade cinética em experimentos no modo potenciostático e galvanostático $[62,63]$. Especificamente no caso do metanol as etapas reacionais têm sido extensivamente discutidas na literatura [98] e sua complexidade se deve à variedade de intermediários adsorvidos na superfície do eletrodo, que pode ser $P t$ monocristalina ou policristalina [99]. Os mecanismos para o metanol discutido na literatura são conhecidos por associar dois caminhos paralelos acontecendo simultaneamente que podem ser formulados como: (a) uma via dita indireta causada pela adsorção do monóxido de carbono na superfície envolvendo várias etapas de desidrogenação da molécula e (b) uma via direta que envolve a adsorção da molécula de metanol pelo átomo de carbono formando monóxido de carbono que reage com espécies hidrogenadas na superfície de $\mathrm{Pt}$ formando $\mathrm{CO}_{2}$ [100].

Um ponto a ser destacado aqui, com relação ao comportamento observado durante a eletro-oxidação dessas moléculas, é o fato das séries temporais obtidas em experimentos apresentarem uma variação em seu comportamento associada ao tempo de oscilação chamada de drift. Tal variação é conhecida por relacionada uma dependência temporal com um ou mais parâmetros livres no sistema afetando de forma significativa sua dinâmica. A hipótese relacionada a esse comportamento é que o sistema pode ser descrito por dois subsistemas envolvendo duas escalas de tempo distintas [101], onde teríamos um subsistema com uma escala de tempo lenta que está relacionado à variação dos parâmetros livres no tempo definido por $\mathbf{g}$ e um subsistema rápido q que seria influenciado por $\mathbf{g}$ causando uma variação da série temporal no tempo. Nesse caso, o sistema de equações que descreve o comportamento para dados experimentais com as duas escalas de tempo pode ser descrito por conjunto vetorial: 


$$
\begin{gathered}
\frac{d \mathbf{q}}{d t}=\mathbf{f}(\mathbf{q}, \gamma(\mathbf{g}), t) \\
\frac{d \mathbf{g}}{d t}=\kappa \mathbf{h}(\mathbf{g}, \mathbf{q}, t)
\end{gathered}
$$

onde, $\mathbf{q} \in \mathbb{R}^{n}$ corresponde a direção observada nos experimentos e corresponde ao sistema rápido. Já $\mathbf{g} \in \mathbb{R}^{m}$ refere-se ao sistema lento escondido no processo dinâmico do sistema e $\gamma \in \mathbb{R}^{s}$ é uma função de $\mathbf{g}$ que representa um ou mais parâmetros no sistema. Por fim o termo $\kappa$ que corresponde ao parâmetro que mede a influência do subsistema lento.

É importante salientar que em experimentos eletroquímicos a variação dos parâmetros livres possa esta associada à variação dos períodos nas séries temporais. De fato, Okamoto et al. [102] mostram que o mecanismo de oscilação envolvendo a variação do período deve ser causado pelo acúmulo ou consumo de espécies adsorvidas no eletrodo. Contudo, o problema esta em determinar os parâmetros que podem estar associado ao comportamento da série temporal e associar com possíveis espécies presente no mecanismo de reação. Tal proposta de estudo será levantada nessa parte da Tese para o metanol.

Uma das formas utilizadas para se determinar o número de parâmetros em um sistema experimental consiste no uso das ferramentas de análise estatística conhecidas como análise fatorial [103]. Essa ferramenta reduz a dimensão das matrizes de dados em dimensões menores, por meio do uso de espaços de fatores ortogonais e transformações que auxiliam na predição e reconstrução dos dados. Entretanto, essa ferramenta não pode ser utilizada diretamente nas séries temporais. Para utilizar tal ferramenta, se faz necessário separar as contribuições dos dois subsistemas. Nesse caso temos que fazer uso de outra ferramenta chamada de espaço de fase alterado, do inglês phase-space warping (PSW), desenvolvido por Chelidze e colaboradores [104-107]. Essa metodologia monitora o espaço de fase por meio de uma regressão linear multivariada local [108] gerando uma matriz $\mathbf{M}$ que corresponde à aglomeração sequenciada dos vetores de monitoramento, obtidos para o ajuste do modelo linear multivariado. De posse da matriz de monitoramento $\mathbf{M}$, podemos aplicar todas as ferramentas de análise multivariada descrevendo o comportamento do subsistema lento. 


\section{II.2 Objetivos}

O objetivo desta segunda parte da Tese é propor uma metodologia sistemática para o tratamento das variações dos parâmetro com o tempo observados em experimentos eletroquímicos. Para esse estudo, foi obtido uma série temporal experimental em condições galvanostáticas para a reação de eletro-oxidação de metanol sobre platina. Ainda que aplicado a um experimento específico, a metodologia desenvolvida é de validade geral. 


\section{II.3 Descrição da Metodologia}

\section{II.3.1 Obtenção da Série Temporal}

A série temporal analisada nessa Tese consiste de uma parte dos resultados obtidos para a eletro-oxidação do metanol em platina policristalina. A metodologia utilizada para obtenção desse resultado pode ser encontrada no trabalho de Nagao, Sitta e Varela [109]. Nesse trabalho foi apresentado um método empírico para estabilizar séries temporais experimentais de potencial variando no tempo.

\section{II.3.2 Tratamento da Série Temporal}

Como comentado anteriormente temos que o sistema dinâmico pode ser visto como um conjunto de dois subsistemas comportando-se em escalas de tempos diferentes. Essa diferença causa uma variação nos parâmetros do sistema, resultado em um drift nas séries temporais.

Dada uma série temporal correspondendo ao subsistema rápido que pode ser representado por $x(n)$, podemos reconstruir um espaço de fase equivalente ao sistema dinâmico por meio do método de Takens descrito na literatura [110-112] e dado por:

$$
\mathbf{y}_{n}=[x(n), x(n+\tau), \ldots, x(n+(d-1) \tau)]^{T}
$$

onde $\mathbf{y}_{n} \in \mathbf{R}^{d}$ corresponde ao espaço de fase associado à série temporal, $\tau$ refere-se ao tempo de atraso e $d$ representa a dimensão do espaço de fase reconstruído. Esse método é muito interessante, pois permite obter informações internas ao sistema que não são acessadas apenas observando a série temporal. Contudo, uma das grandes vantagens é o fato de podermos associar a esse espaço de fase reconstruído um mapa definido por: 


$$
\mathbf{y}_{n+1}=\mathbf{P}\left(\mathbf{y}_{n} ; \mathbf{g}\right)
$$

que segundo Chelidze et al. [101] é distorcido pelo drift causado pelo subsistema lento. O princípio do método $P S W$ consiste em ajustar um modelo linear multivariado a esse mapa e gerar um vetor de monitoramento que é utilizado para formar uma matriz $\mathbf{M}$ (matriz de monitoramento). Nesse método, estamos assumindo que todas as variáveis que atuam no drift do sistema estão presentes nessa matriz, assim como o erro experimental que deve ser considerado como muito pequeno em relação ao sinal de monitoramento.

Após as considerações acima, o problema agora se resume em determinar quais variáveis são realmente relevantes para explicar o comportamento de M. Para isso foram utilizadas ferramentas multivariadas de análise conhecidas como análise dos fatores. A base fundamental na análise multivariada consiste em analisar os autovalores referentes a uma matriz de dados D, onde podemos ver os dados como um produto matricial de duas matrizes, uma matriz linha chamada de $\mathbf{B}$ e uma matriz coluna representada por $\mathbf{C}$, o produto matricial é mostrado na Equação 44.

$$
\mathbf{D}_{n x m}=\mathbf{B}_{n x p} \mathbf{C}_{p x m}
$$

De acordo com o Malinowski [103] a matriz D pode ser vista também com o produto matricial entre três matrizes dadas por:

$$
\mathbf{D}_{n x m}=\mathbf{U}_{n x p} \mathbf{S}_{p x p} \mathbf{V}_{p x m}^{\prime}
$$

nesse caso as matrizes $\mathbf{B}$ e $\mathbf{C}$ são definidas como:

$$
\begin{gathered}
\mathbf{B}_{n x p}=\mathbf{U}_{n x p} \mathbf{S}_{p x p} \\
\mathbf{C}_{p x m}=\mathbf{V}_{p x m}^{\prime}
\end{gathered}
$$

onde a matriz $\mathbf{S}$ refere-se à matriz diagonal e seus elementos são as raízes quadradas dos autovalores representada pela matriz diagonal $\Lambda$. Um ponto importante a ser ressaltado aqui é que toda a estatística utilizada na análise dos fatores se baseia no tratamento do vetor diagonal $\operatorname{de} \Lambda$. 


\section{II.4 Resultados e Discussão}

O resultado típico obtido no experimento galvanostático pode ser visualizado na Figura 29 (a) para o valor de densidade de corrente $1,00 \mathrm{~mA} \mathrm{~cm}^{-2}$ [109]. Note que existe uma região ampla na série apresentando oscilações harmônicas com seus limites indicados pelo retângulo azul, limite esquerdo da série e vermelho referente ao limite direito. Esses limites são mostrados nas Figuras 29 (b) e (c) respectivamente. Observe-se também que a série temporal contempla uma ampla faixa de padrões oscilatórios com períodos de ordem $1^{0}, 1^{1}, 1^{2}, 1^{3}, \ldots, 1^{n}$ seguindo um ramo de sequência da árvore de Stern-Brocot, ver Figura 27 referente à sequência do ramo $A$ em azul.
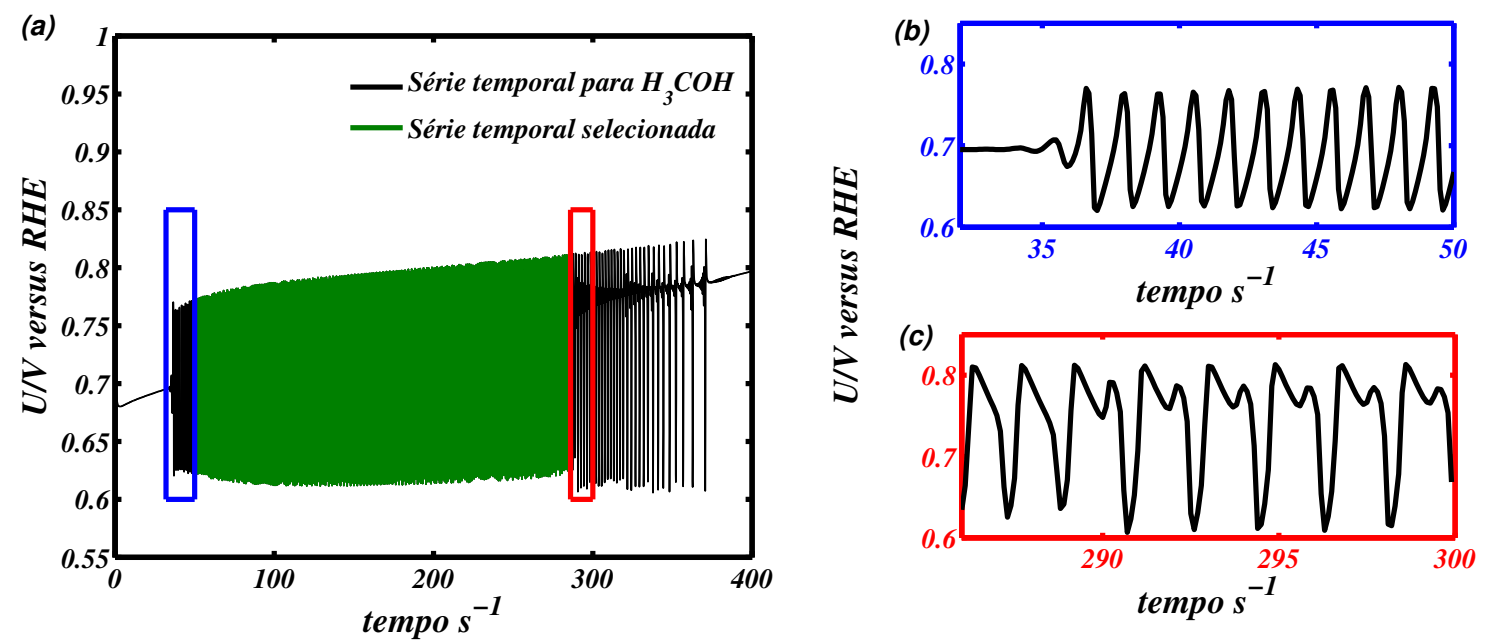

Figura 29: (a) Série temporal para a eletro-oxidação do metanol à $0,5 \mathrm{ml} \mathrm{L}^{-1}$ obtida para a densidade de corrente $1,00 \mathrm{~mA} \mathrm{~cm}^{-2}$, onde (b) e (c) correspondem às extremidades da região utilizada na análise multivariada. 
Para analisar essa série temporal foi selecionada uma região com um tipo de período dominante. Tal região é indicada pela cor verde na Figura 29 (a). Um ponto importante a ser ressaltado é que o método $P S W$ não permite a comparação entre regiões com períodos diferentes. O motivo dessa limitação é que na utilização desse método se faz necessário reconstruir o espaço de fase para um sistema experimental usando o método de Takens ou método do atraso, para isso é necessário determinar o tempo de atraso ideal definido por $\tau_{\text {ideal }}$, que pode mudar para regiões com períodos diferentes. Nesse caso, tem-se que para cada região da série temporal dominada por um período distinto um espaço de fase diferente. Além disso, a determinação do $\tau_{\text {ideal }}$ é de fundamental importância para determinar o melhor espaço de fase reconstruído.

Uma das formas para se determinar o $\tau_{\text {ideal }}$ é utilizar a função de informação mútua para a região selecionada na Figura 29 (a). Esse método é baseado na teoria da informação de Shannon e consiste na medida das entropias para o sistema estudado [113]. Para se determinar o $\tau_{i d e a l}$ deve-se levar em consideração o primeiro mínimo do gráfico de informação mostrado na Figura 30.

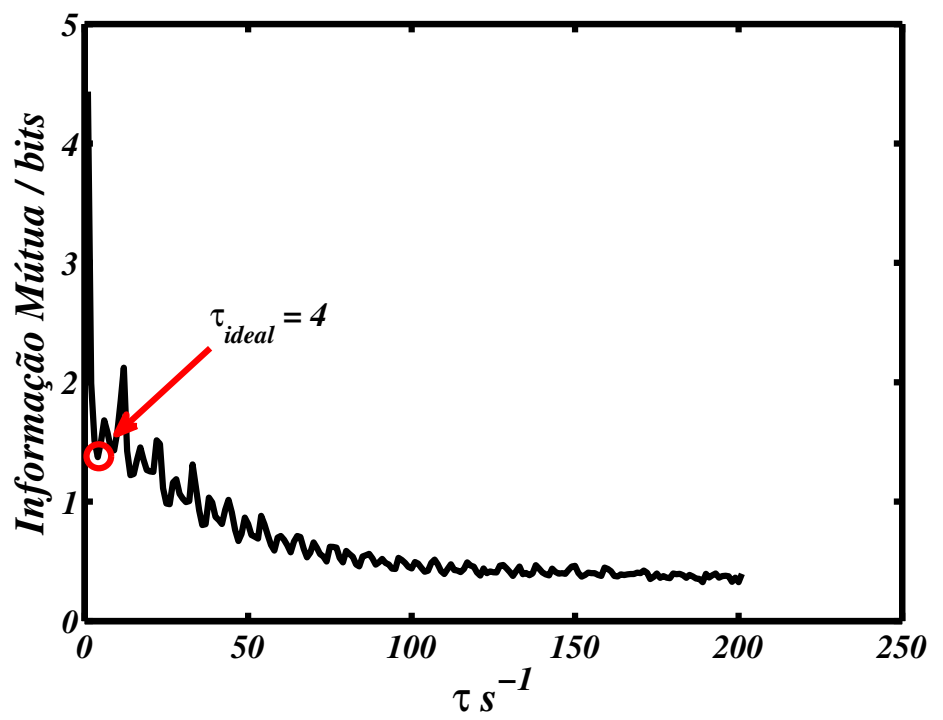

Figura 30: Função de informação mútua para a série temporal selecionada na Figura 29 (a), onde a seta em vermelho indica a posição do primeiro mínimo da função e o valor do $\tau_{\text {ideal }}=4$. 
A Figura 30 fornece o valor do $\tau_{\text {ideal }}=4$ que é utilizado na reconstrução do espaço de fase otimizado, dessa forma pode-se construir uma matriz de dados chamada de $\mathbf{D}$. A matriz correspondente ao espaço de fase reconstruído é mostrada na Figura 32 (a), onde o eixo $x$, irá corresponder às linhas na matriz $\mathbf{D}$, refere-se ao tempo de cada segmento. Já o eixo $y$ representa os elementos nas colunas da matriz $\mathbf{D}$ e refere-se às dimensões do espaço de fase. Outro ponto importante e que deve ser levado em consideração durante a reconstrução do espaço de fase, são os falsos vizinhos próximos (do inglês False Nearest Neighbors, FNN) [110]. Um FNN corresponde a um ponto num conjunto de dados que é vizinho apenas porque a órbita reconstruída no espaço de fase esta contraída, esses pontos podem causar problemas no tratamento referente ao ajuste do modelo linear multivariado. Para determinar a influência dos $F N N$ foi calculada as dimensões de embutimento (do inglês embedding dimension, ED), como mostrado na Figura 31.

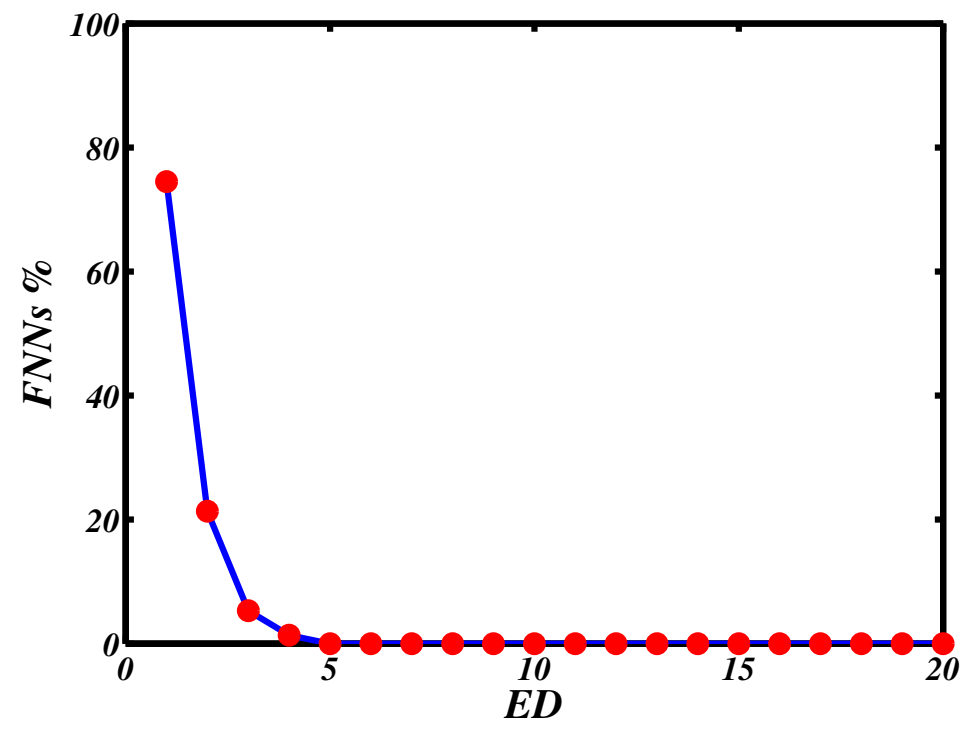

Figura 31: Dimensão ED para a série temporal selecionada na Figura 29 (a).

De acordo com a Figura 31 temos que o valor de $E D$ adequado, levam em consideração uma porcentagem pequena de $F N N$ e uma convergência na rotina $P S W$ fornecida por Chelidze [114], foi o valor de $E D=3$. Utilizando os valores de $\tau_{\text {ideal }}=4$ e $E D=3$, foi calculada uma matriz de monitoramento $\mathbf{M}$ com dimensão 150. O número de pontos usado na modelagem local foi de 150 e o resultado pode ser observado na Figura 32. 

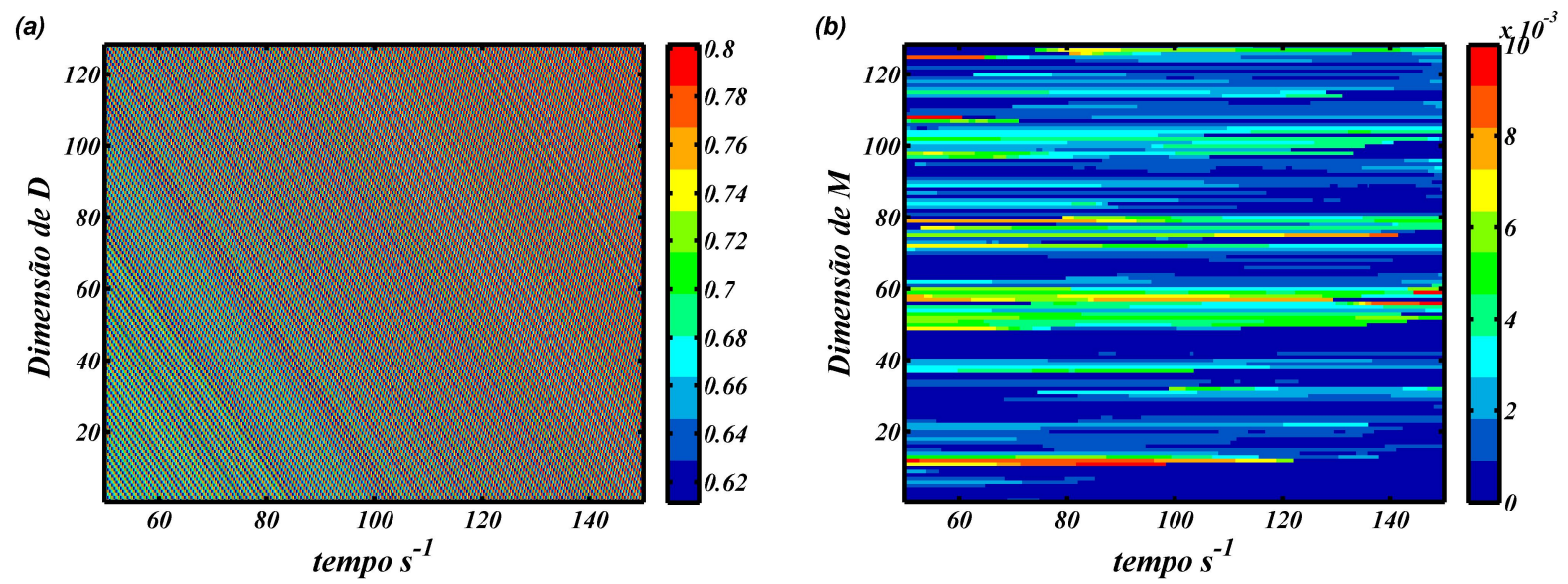

Figura 32: (a) Espaço reconstruído pelo método de Takens com $\tau_{\text {ideal }}=4$ e (b) Matriz de monitoramento obtida pelo método $P S W$ com dimensão incorporada de $d=3$.

Uma vez obtida a matriz $\mathbf{M}$ o procedimento foi calcular os fatores usando a decomposição dos valores singulares $(S V D)$ que gera a relação exibida na Equação 45. Esse procedimento foi realizado para obter a matriz dos autovalores referente à matriz de monitoramento, um gráfico do $\log (\Lambda)$ pode ser visto na Figura 33 (a). A análise dos autovalores é de fundamental importância, pois as intensidades refletem a relação sinal/ruído existente nos dados experimentais. Essa relação separa os autovalores em dois subconjuntos que serão chamados aqui de autovalores principais e autovalores secundários. Os autovalores principais têm com característica estarem associados ao número de variáveis no sistema. Já os secundários são atribuídos ao erro experimental. Contudo, quando observamos a Figura 33 (a) não é possível inferir o número de variáveis no sistema referente ao comportamento da série temporal. Para auxiliar na análise foi utilizada uma função empírica desenvolvida por Malinowski [103] chamada de Função Indicadora de Fator (do inglês Factor Indicador Function, IND), essa função é definida pela Equação 48.

$$
\begin{gathered}
I N D=\frac{R E}{(c-n)^{2}} \\
R E=\left[\frac{\sum_{j=n+1} \lambda_{j}^{0}}{r(c-n)^{2}}\right]^{\frac{1}{2}}
\end{gathered}
$$


em que $R E$ corresponde ao erro real da medida, $r$ representa o número de linhas da matriz $\mathbf{M}$, $c$ refere-se ao número de colunas de $\mathbf{M}$ e $n$ diz respeito ao número do autovalor considerado no laço para calcular os vetores $I N D$ e $R E$. Essa função tem como característica principal aproxima-se rapidamente do limite entra os autovalores principais e secundários, fornecendo um valor que corresponde ao máximo de variáveis possíveis no sistema. Pode-se observar um gráfico da função IND na Figura 33 (b), onde o ponto em vermelho indica o mínimo da função que corresponde a 58 variáveis no sistema.
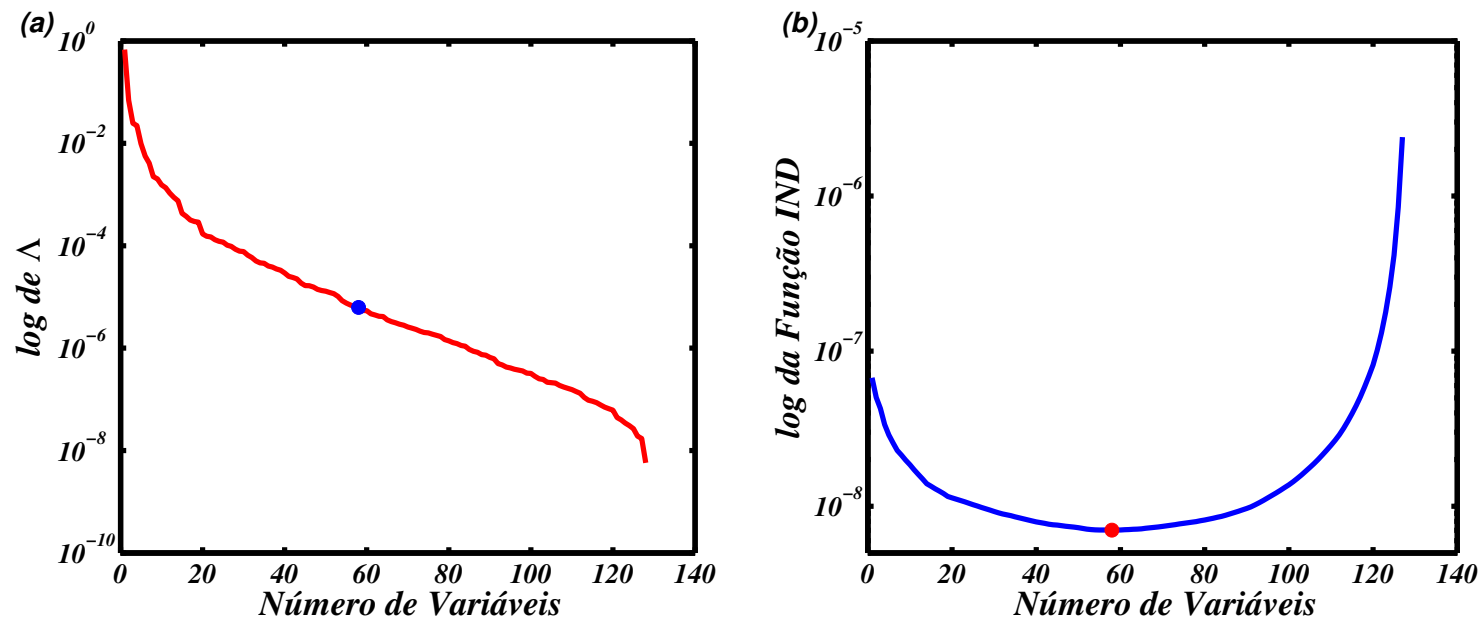

Figura 33: (a) Gráfico dos autovalores em função do números de fatores na matriz M. Já (b) corresponde ao gráfico da função IND definida por Malinowski [103].

Apesar do máximo número de variáveis indicado pela função $I N D$, ainda assim é um valor relativamente alto para ser considerado quando queremos descrever um comportamento experimental num modelo mais simples possível. Para minimizar o número de variáveis do sistema foi realizada uma série de estudos estatísticos nos autovalores, os resultados podem ser vistos na Tabela 6, onde $R E V$ representa o erro reduzido dos autovalores que pode ser associado ao erro na obtenção dos autovalores da matriz de dados, \%NS corresponde à porcentagem do nível de significância dos autovalores sendo calculado por meio do teste $F$ dado pela Equação 51. Por fim, temos a percentagem da variância acumulada \%VA referente aos autovalores e definida pela Equação 54, esse parâmetro é de fundamental importância nessa análise pois ele informa o quanto podemos explicar dos dados experimentais com base no número dos autovalores. Todas as Equações utilizadas na análise são mostradas abaixo: 


$$
\begin{gathered}
R E V_{j}=\frac{\lambda_{j}}{(r-j+1)(c-j+1)} \\
F(1, s-n)=\frac{\sum_{j=n+1}^{s}(r-j+1)(c-j+1)}{(r-n+1)(c-n+1)} \frac{\lambda_{n}}{\sum_{j=n+1}^{S} \lambda_{j}^{0}} \\
N S=100(1-F) \\
V_{j}=\frac{\lambda_{j}}{\sum_{j=1}^{c} \lambda_{j}} \\
V A_{j}=100 \sum_{j=1}^{n} V_{j}
\end{gathered}
$$

onde o termo $s$ refere-se ao menor valor entre $r$ e $c$. Já o termo $j$ representa o número do elementos analisado no laço do programa. O parâmetro $V_{j}$ na Equação 53, corresponde a variância para cada autovalor.

Tabela 6: Parâmetros estatísticos obtidos para autovalores da matriz $\mathbf{M}$

\begin{tabular}{ccccccc}
\hline$n$ & $\lambda$ & $R E$ & $I N D$ & $R E V$ & $\% N S$ & $\% V A$ \\
\hline & & & & & & \\
1 & 0,6806 & 0,0011 & $6,73 \times 10^{-8}$ & $5,32 \times 10^{-6}$ & 0 & 81,98 \\
2 & 0,0693 & $7,98 \times 10^{-4}$ & $5,03 \times 10^{-8}$ & $5,47 \times 10^{-7}$ & $4,34 \times 10^{-9}$ & 90,33 \\
3 & 0,0248 & $6,66 \times 10^{-4}$ & $4,26 \times 10^{-8}$ & $1,97 \times 10^{-7}$ & $5,26 \times 10^{-5}$ & 93,32 \\
4 & 0,0222 & $5,18 \times 10^{-4}$ & $3,37 \times 10^{-8}$ & $1,78 \times 10^{-7}$ & $4,93 \times 10^{-7}$ & 95,99 \\
5 & 0,0099 & $4,36 \times 10^{-4}$ & $2,88 \times 10^{-8}$ & $8,00 \times 10^{-8}$ & $1,66 \times 10^{-4}$ & 97,18 \\
6 & 0.0056 & $3,81 \times 10^{-4}$ & $2,56 \times 10^{-8}$ & $4,61 \times 10^{-8}$ & 0,0033 & 97,86 \\
7 & 0.0041 & $3,36 \times 10^{-4}$ & $2,29 \times 10^{-8}$ & $3,39 \times 10^{-8}$ & 0,0055 & 98,36 \\
8 & 0,0022 & $3,08 \times 10^{-4}$ & $2,14 \times 10^{-8}$ & $1,86 \times 10^{-8}$ & 0,1029 & 98,63 \\
9 & 0,0020 & $2,81 \times 10^{-4}$ & $1,98 \times 10^{-8}$ & $1,70 \times 10^{-8}$ & 0,0641 & 98,87 \\
10 & 0,0015 & $2,58 \times 10^{-4}$ & $1,85 \times 10^{-8}$ & $1,31 \times 10^{-8}$ & 0,1122 & 99,06 \\
11 & 0,0014 & $2,35 \times 10^{-4}$ & $1,72 \times 10^{-8}$ & $1,16 \times 10^{-8}$ & 0,0829 & 99,22 \\
$\vdots$ & $\vdots$ & $\vdots$ & $\vdots$ & $\vdots$ & $\vdots$ & $\vdots$ \\
58 & $6,23 \times 10^{-6}$ & $3,44 \times 10^{-5}$ & $7,02 \times 10^{-9}$ & $9,31 \times 10^{-11}$ & 11,3414 & 99,99 \\
$\vdots$ & $\vdots$ & $\vdots$ & $\vdots$ & $\vdots$ & $\vdots$ & $\vdots$ \\
\hline
\end{tabular}


Quando observamos a Tabela 6, percebe-se que a variância acumulada para os primeiros três autovalores representa 93,32\% dos dados experimentais. Entretanto, se considerarmos os cinco primeiros autovalores explicaremos cerca de 97,18\%. Contudo, vale lembra na medida em que aumentamos o número de variáveis para explicar os dados experimentais estamos também aumentando o grau de complicação do modelo mecanístico. Nesse caso o número de variáveis adotado deve satisfazer uma condição, que é explicar mais de 90,00\% dos daods experimentais.

Outro ponto importante é que experimentos eletroquímicos acoplados com espectroscopia de infravermelho apontam para uma variável presente durante a eletro-oxidação do metanol diferente do $C O_{a d}$ [115]. Bocheto et al. [62] mostraram usando a técnica de $A T R-S E I R A S$ (do inglês surface enhanced infrared absorption spectroscopic in attenuated total reflection configuration) que durante o comportamento oscilatório do metanol os recobrimentos na superfície de $P t$ sofrem alterações no tempo. Os autores mostram que a integração da banda de $C O_{L}$ diminui com o tempo de oscilação, eles também revelaram a existência de uma banda referente ao formiato $(\mathrm{HCOO})$ presente no processo. Por fim, o trabalho de Nagao, Sitta e Varela [109] mostrou que uma compensação na densidade de corrente, pode mudar o tempo de duração para um tipo específico de período existente na série temporal. Eles descreveram um cenário mecanístico simplificado para a variação no recobrimento superficial por meio de três espécies que atuariam na eletro-oxidação do metanol, as espécies propostas foram o $\mathrm{CO}, \mathrm{HCOO}$ e $\mathrm{OH}$.

Com base no relato experimental foi assumido o número de três variáveis para descrever o comportamento do drift existente no sistema experimental. Os respectivos autovetores para as matrizes $\mathbf{B}$ e $\mathbf{C}$ referentes aos três primeiros autovalores são mostradas nas Figuras 34 (a) e (c) respectivamente. Um ponto curioso aqui é que apesar de podemos descrever 93,32\% dos dados, os autovetores não têm o compromisso em dar significado físico as variações observadas, isso faz com que alguma comparação com resultados experimentais se torne impossível. Contudo, é possível rotacionar aos autovetores por meio de uma matriz rotacional chamada T, essa aproxima os autovalores a uma dimensão matemática com características similares à dimensão física. Tal metodologia é conhecida como Resolução de Curvas Multivariadas (do inglês Multivariate Curve Resolution, MCR) [116-119]. Na utilização do método MCR foi usa- 
da a rotina $m c r f m i n$ desenvolvidas por Tauler [120], os resultados podem ser observados nas Figuras 34 (b) e (d).
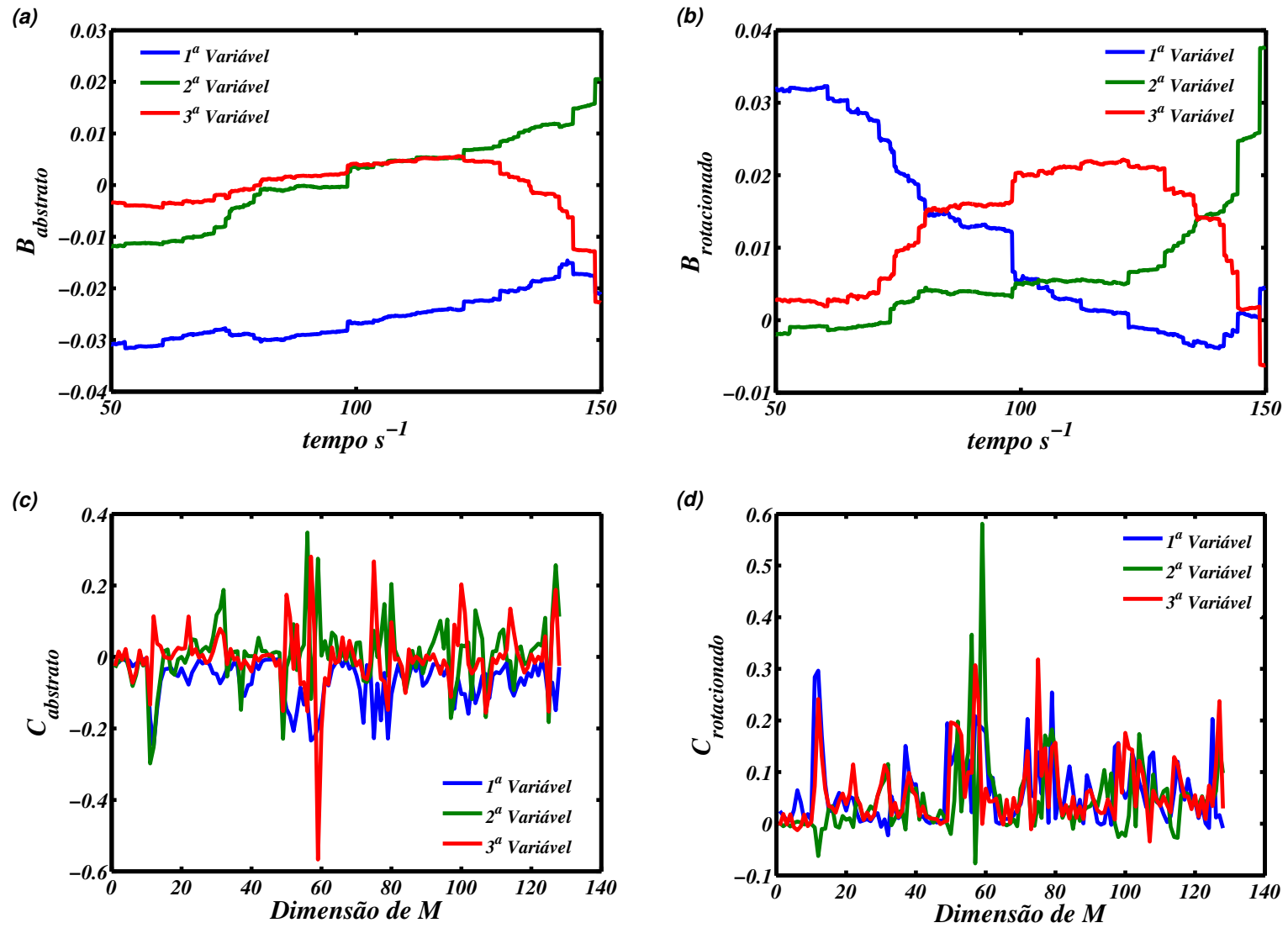

Figura 34: Autovetores correspondentes à matriz $\mathbf{M}$, onde (a) e (c) correspondem às matrizes $\mathbf{B}$ e $\mathbf{C}$ sem rotação no espaço de variáveis. Já os gráficos (b) e (d) referem-se às matrizes $\mathbf{B}$ e $\mathbf{C}$ rotacionadas no espaço por meio da matriz de rotação $\mathbf{T}$.

Observando a Figura 34 (b), percebe-se que as rotações dos autovetores da matriz B possuem uma similaridade com perfis de concentração variando no tempo. Observe que a primeira variável indicada pela linha azul, diminui com o tempo e pode ser relacionada com a variação do recobrimento de $C O_{a d}$. Já a segunda variável representada pela linha verde, pode ser atribuída ao comportamento do $\mathrm{OH}_{a d}$ que representa as espécies hidrogenadas, essa por sinal aumenta à medida que o $C O_{a d}$ diminui. Tal comportamento é esperado, pois o $C O_{a d}$ reage com $\mathrm{OH}_{a d}$ formando $\mathrm{CO}_{2}$. Contudo, quando olhamos para a terceira variável notamos um comportamento de intermediário que pode ser relacionado ao formiato $H C O O_{a d}$ na reação de eletro-oxidação. Um ponto importante aqui é o fato de que todas essas variáveis foram extraídas com relação ao drift da série temporal referente à reação de eletro-oxidação do metanol. Isso 
leva a crer que as variações existentes na superfície em relação aos recobrimentos podem estar relacionadas diretamente com o fenômeno do drift e que existe três espécies envolvidas com perfis de concentração próximos ao mostrado na Figura 34 (b). 


\section{II.5 Conclusões}

Apresentou-se nessa parte da Tese um estudo sistemático do drift referente a série temporal obtida para a reação de eletro-oxidação do metanol, e com base nas análises estatísticas foi demonstrado que $93,32 \%$ do comportamento responsável pelo drift pode ser explicado com base em três variáveis, que podem estar diretamente envolvidas nos processos que dominam o comportamento do recobrimento na superfície do eletrodo. Outro ponto discutido foi a obtenção dos perfis com relação às três variáveis consideradas, que podem ser associados aos perfis experimentais sugeridos na literatura $[62,109,115]$. 


\section{Conclusões Gerais}

Dois diferentes aspectos associados às instabilidades cinéticas observadas em sistemas eletroquímicos foram abordadas nessa Tese. Apresentou-se inicialmente um estudo criterioso do modelo HN-NDR por meio dos diagramas de estabilidade, de Lyapunov e de período, onde foi observada a formação de padrão auto-organizado chamados de shrimps no interior da bifurcação de Hopf. Observou-se que a dinâmica interna é influenciada pelos pontos de sela-foco, referentes à condição de Shilnikov, assim como os pontos de sela-nó por meio do princípio de reinjeção comprovando a existência de duas regiões com escalas de tempo bem definidas no modelo. Um outro ponto relatado na primeira parte da Tese é com relação ao estudo realizado por meio dos diagramas de período que descreveu um comportamento periódico explicado pela sequência de Stern-Brocot. Já na segunda parte dessa Tese foi estudada uma série temporal obtida para a reação de eletro-oxidação do metanol na superfície de Pt policristalina por meio da análise multivariada. Nesse estudo foi demonstrado que o drift apresentado pela série pode ser descrito por três variáveis que pode ser atribuídas às seguintes espécies $\mathrm{CO}_{a d}, \mathrm{HCOO}_{a d}$ e $\mathrm{OH}_{a d}$. 


\section{Perspectivas}

Observou-se nessa Tese o comportamento dinâmico para o conjunto de Equações 22, 23 e 24 que descreve o comportamento do modelo no modo potenciostático. Contudo, pouco foi feito em relação ao modelo galvanostático obtido pela substituição do segundo termo da Equação 22 por $I$. Uma curiosidade aflora quando questionamos a respeito do possível comportamento apresentado pelos diagramas de estabilidade, de Lyapunov e de período. Uma interessante questão é se podemos observar comportamento homoclínico de sela-foco e sela-nó que poderia gerar padrões como shrimps ou simplesmente não é observado comportamento homoclínico de sela-foco e sela-nó gerando a inexistência de padrões auto-organizados no interior da bifurcação.

Outro ponto que levantamos aqui é a necessidade de fazer diagramas de estabilidade para outros modelos diferentes para poder evidenciar a real contribuição das condições de Shilnikov. Sabemos que podem existir condições diferentes que apresentem um comportamento dinâmico rico com formações de padrões auto-organizados. Contudo, ainda é incerto se esses padrões são formados por condições que não apresentem comportamento homoclínico associado ao sistema. Aparentemente se observa que sempre que o sistema apresenta esses comportamentos tem um comportamento homoclínico associado a ele.

Continuando em nossa perspectiva, seria interessante realizar um estudo levando em consideração a sequência da árvore de Stern-Brocot para descrever o comportamento existente em séries temporais experimentais, como por exemplo, a série da Figura 29 (a) que possui uma sequência dada por $1^{0}, 1^{1}, 1^{2}, 1^{3}, \ldots, 1^{n}$. Observe que essa sequência corresponde a um ramo da árvore de Stern-Brocot referente à sequência $A$ em azul. Tal característica é extremamente interessante uma vez que podemos associar o comportamento periódico de uma série temporal experimental a uma sequência numérica, essa característica faz com que muitas das sequências observadas possam ser explicadas pela árvore de Stern-Brocot. Pensando nisso levantamos um 
ponto interessante, é possível que muitos dos comportamentos periódicos observados e que sejam de difícil entendimento possam ser explicada imaginando cada ramo da árvore como um nó, que pode esta ligada a outros nós localizados em diferentes ramos da árvore. Nesse caso, para que o sistema possa atingir o outro ramo ele necessariamente teria que passar pelos períodos existentes entre esses nós. Caso o sistema venha apresentar comportamento caótico, isso implicaria que ao invés de passar por todos os períodos existentes entre as duas extremidades, ele simplesmente saltaria alguns períodos. Essa idéia levanta a possibilidade de associar a árvore de Stern-Brocot a uma rede (network) e que cada nó funcionaria como um $H u b$ comunicando todos os períodos no sistema. A pergunta que fazemos aqui é de que maneira podemos relacionar esses nós para obter uma sequência compatível com o comportamento experimental? Do ponto de vista químico, se representamos cada nó como uma etapa reacional sua sequência significaria o mecanismo reacional global?

Um outro ponto crucialmente importante relatado nessa Tese foi à influência do drift apresentado pela série temporal da reação de eletro-oxidação do metanol. Foi constatado utilizando a análise multivariada que podemos relacionar o drift a diversas variáveis atuando na superfície do eletrodo e explicar uma parte do comportamento. Nesse ponto seria muito interessante aplicar tal metodologia no tratamento de diversas séries temporais para auxiliar na formulação dos mecanismos. 


\section{Referências Bibliográficas}

1. WOJTOWICZ, J., Oscillatory Behavior in Electrochemical Systems, Modern Aspects of Eletrochemistry No.8, J. O’M. Bockris and B. E. Conway, p. 47, Ed. Plenum Press New York, 1972.

2. KOPER, M. T. M., Oscilations and Complex Dynamical Bifurcations in Electrochemical Systems, Advances in Chemical Physics V. 92, I. Prigogine and S. A. Rice, p. 161, Ed. John Wiley \& Sons, 1996.

3. KEPPER, P. and EPSTEIN, I. R., A Mechanistic Study of Oscillations and Bistability in the Briggs-Rouscher Reaction, Journal of the American Chemical Society, v. 104, p. 49-55, 1982.

4. OBÁN, M.; KEPPER, P. and EPSTEIN, I. R., Minimal Bromat Oscillator Bromate-Bromite-Catalyst, Journal of the American Chemical Society, v. 104, p. 2657-2658, 1982.

5. KEPPER, P.; EPSTEIN, I. R.; KUSTN, K. and OBÁN, M., Batch Oscillations and Spatial Wave Patterns in Chlorite Oscilating Systems, Journal of the American Chemical Society, v. 82, p. $170-171,1982$.

6. TURNER, J. S.; ROUX, J. C.; McCORMICK, W. D. and SWINNEY, L., Alternating Periodic and Chaotic Regimes in a Chemical Reaction - Experiment and Theory, Physics Letters, v. 85 A, p. 9-12, 1981.

7. HUDSON, J. L.; HART, M. and MARINKO, D., An experimental study of multiple peak periodic and nonperiodic oscillations in the Belousov-Zhabotinskii reaction, Journal of Chemical Physics, v. 71, p. 1601-1606, 1979.

8. HUDSON, J. L. and TSOTSIS, T. T., Electrochemical Reaction Dynamics: A Review, Chemical Engineering Science, v. 49, p. 1493-1572, 1994.

9. BASSETT, M. R. and HUDSON, J. L., Quasi-Periodicity and Chaos during an Electrochemical Reaction, Journal of Physics Chemical, v. 93, p. 2731-2737, 1989.

10. ALBAHADILY, F. N.; RINGLAND, J.; SCHELL, M., Mixed-mode Oscillation in an Electrochemical System. I. A farey sequence which does not occur an a torus, Journal of Chemical Physics, v. 90, p. 813-821, 1989.

11. SCHELL, M. and ALBAHADILY, F. N., Mixed-mode Oscillations in an Electrochemical System. II. A Periodic-Chaos Sequence, Journal of Chemical Physics, v. 90, p. 822-828, 1989. 
12. AlligOOD, K. T.; SAUER, T. D.; YORKE, J. A., Chaos an Introduction to Dynamical Systems, Springer, 1996.

13. STROGATZ, S. H., Nonlinear Dynamics and Chaos: With Applications to Physics, Biology, Chemistry, and Engineering. Addison-Wesley, 1994.

14. SEYDEL, R., Practical Bifurcation and Stability Analysis, Springer, 2010.

15. HILBORN, R. C., Chaos and Nonlinear Dynamics: An Introduction for Scientists and Engineers, Oxford, 1994.

16. KOPER, M. T. M.; SLUYTERS, J. H., A mathematical model for current oscillations at the active-pasive transition in metal electrodissolution, Journal Electroanalytical Chemistry, v. 347, p. 31-48, 1993.

17. SAZOU, D.; PAGITSAS, M.; GEORGOLIOS, C., Bursting and Beating Current Oscillatory Phenomena Induced by Chloride Ions During Corrosion/Passivation of Iron in Sulphuric Acid Solutions, Electrochimica Acta, v. 38, p. 2321-2332, 1993.

18. SAMJESKÉ, G.; OSAWA, M., Current Oscillations during Formic Acid Oxidation on Pt Eletrode: Insight into the Mechanism by Time-Resolved IR Spectroscopy, Angewandte Chemie, v. 44, p. 5694-5698, 2005.

19. KRISCHER, K., Prnciples of Temporal and Spatial Pattern Formation in Electrochemical Systems, Modern Aspects of Eletrochemistry No.32, J. O’M. Bockris , B. E. Conway and R. E. White, p. 1, Ed. Kluwer Academic Publishers New York, 2002.

20. KOPER, M. T. M.; SLUYTERS, J. H., Electrochemical Oscillators: Their Description Through a Mathematical Model, Journal Electroanalytical Chemistry, v. 303, p. 73-94, 1991.

21. STRASSER, P.; EISWIRTH, M.; KOPER, M. T. M., Mechanistic Classification of Electrochemical Oscillators - An Operational Experimental strategy, Journal Electroanalytical Chemistry, v. 478, p. 50-66, 1999.

22. VARELA, H.; KRISCHER, K., Nonlinear Phenomena during Electrochemical Oxidation of Hydrogen on Platinum Electrodes, Catalysis Today, v. 70, p. 411-425, 2001.

23. VARELA, Hamilton. Spatiotemporal Patterm Formation during Electrochemical Oxidation of Hydrogen on Platinum. 2003. $170 \mathrm{f}$. Ph. D. Thesis, Freie Universität Berlin, http://www.diss.fu-berlin.de/2003/304/indexe.html 003.

24. KRISCHER, K; VARELA, H., Oscillations and other dynamic instabilities, IN:

Handbook of Fuel Cells: Fundamentals, Technology, Applications. W. Vielstich, H. A. Gasteiger, A. Lamm, v. 2, p. 679, Wiley \& Sons, 2003.

25. CAPON, A.; PARSONS, R., The Oxidation of Formic Acid at Noble Metal Electrodes: I. Review of Previous Work, Journal Electroanalytical Chemistry, v. 44, p. 1-7, 1973. 
26. NAGAO, Raphael. Efeito da Temperatura na Eletro-oxidação Oscilatória de Ácido Fórmico Sobre Platina: Experimentos e Simulações. 2009. 78 f. tese (Mestrado em Físico-Química)-Instituto de Química de São Carlos, Universidade de São Paulo, São Carlos, 2009.

27. SCHELL, M.; ALBAHADILY, F. N.; SAFAR, J.; XU, Y., Characterization of Oscillatory States in the Electrochemical Oxidation of Formaldehyde and Formate/Formic Acid, Journal of Physical Chemistry, v. 93, p. 4806, 1989.

28. SCHELL, M.; XU, Y., Bistability and Oscillations in the Electrocatalyzed Oxidation of Formaldehyde, Journal of Physical Chemistry, v. 94, p. 7137-7143, 1990.

29. MARKOVIC, N. and ROSS, P. N., Oscillatory Behavior in the Electrochemical Oxidation of Formic Acid on Pt(100), Journal of Chemical Physics, v. 97, p. 9771-9778, 1993.

30. STRASSER, P.; LÜBKE, M.; RASPEL, F.; EISWIRTH, M. and ERTL, G., Oscillatory instabilites during formic acid oxidation on $P t(100), P t(110)$ and $\operatorname{Pt}(111)$ under potentiostatic control. I. Experimental, Journal of Chemical Physics, v. 107, p. 979-990, 1997.

31. STRASSER, P.; EISWIRTH, M. and ERTL, G., Oscillatory instabilites during formic acid oxidation on $\operatorname{Pt}(100), \operatorname{Pt}(110)$ and $P t(111)$ under potentiostatic control. II. Model calculations, Journal of Chemical Physics, v. 107, p. 991-1003, 1997.

32. SENSSE, A.; GATERMANN, K.; EISWIRTH, M., Analytic solution for the electrocatalytic oxidation of formic acid, Journal Electroanalytical Chemistry, v. 577, p. 35-46, 2005.

33. SAUERBREI, S; NASCIMENTO, M. A.; EISWIRTH, M. and VARELA, H., Mechanism and model of the oscillatory electro-oxidation of methanol, Journal of Chemical Physics, v. 132, p. 154901-1-10, 2010.

34. KOPER, M. T. M.; SLUYTERS, J. H., On the mathematical unification of a class of electrochemical oscillators and their desing procedures, Journal Electroanalytical Chemistry, v. 352, p 51-64, 1993.

35. KOPER, M. T. M.; SLUYTERS, J. H., Instabilities and Oscillations In Simple-Models of Electrocatalytic Surface-Reactions, Journal Electroanalytical Chemistry, v. 371, p 149-159, 1994.

36. KOPER, M. T. M., The thery of electrochemical instabilities, Electrochimica Acta, v. 37, p. 1771-1778, 1992.

37. BARD, A.; FAULKNER, L. R., Electrochemical Methods Fundamentals and Applications, John Wiley, 2001.

38. BONNEFONT, A.; KOSTECKI, R.; McLARNON, F.; ARRAYET, J. C.; SERVANT, L. and ARGOUL, F., In situ atomic force microscopy imaging of electrodeposition of mixed layers of copper/curous oxide, Journal of the Electroanalitycal Society, v. 146, p. 4101-4104, 1999. 
39. SURVILA, A.; MOCKUS, Z. and JUSKENAS, R., Current oscillations observed during codeposition of copper and tin from sulfate solutions containing Laprol 2402C,

Electrochimica Acta, v. 43, p. 909-917, 1997.

40. TRENIDL, L. and OLEXOVA, A., Electrochemical oscillations of the system $\mathrm{Hg}, \mathrm{HSO}_{4}^{-}$, $\mathrm{BrO}_{3}^{-}$and phenol, Electrochimica Acta, v. 28, p. 1495-1499, 1993.

41. DORFLER, H. D. and MULLER, E., The analusis of current oscillations on the basis of the retardation of electrode processes by different surfactants, Journal of Electroanalytical Chemistry, v. 135, p. 37-53, 1982.

42. LI, Z.; CAI, J.; ZHOU, S., Current oscillations in the reduction or oxidation of same anions involving convection mass transfer, Journal of Electroanalytical Chemistry, v. 436, p. 195-201, 1997.

43. ZHAO, G.; TANG, Y.; CHEN, R.; GENG, R. and LI, D., Potential and current oscillations during formaldehyde oxidation on platinum particles dispersed in three-dimensional pare networks of Tio $_{x} / \mathrm{Ti}$, Elctrochimica Acta, v. 53, p. 5186-5194, 2008.

44. LEVIE, R., On the electrochemical oscillator, Electroanalytical Chemistry and Interfacial Electrochemistry, v. 25, p. 257-273, 1970.

45. LEVIE, R., On some electrochemical oscillators at the mercury I water interface, Journal of Electroanalytical Chemistry, v. 552, p. 223-229, 2003.

46. KARIUKI, S. and DEWALD, H. D., Current oscillations in the reduction of indium (III) and gallium (III) in dilute chloride and nitrate solutions at the dropping mercury electrode, Electrochimica Acta, v. 43, p. 701-704, 1998.

47. KARIUKI, S.; DEWALD, H. D.; THOMAS, J. and ROLLINS, R. W., Current oscillations of indium (III) at a dropping mercury electrode,Journal of Electroanalytical Chemistry, v. 486, p. 175-180, 2000.

48. SCOTT, S. K., Chemical Chaos, v.1, Oxford, 1991.

49. KRISCHER, Katharina, Nonlinear Dynamics in Electrochemical Systems, Advances in Electrochemical Science and Engineering, Richard C. Alkire, v. 8, Wiley, 2002.

50. BONATTO, Cristiano. Estrutura de Diagramas de fase de Sistemas Dinâmicos de Tempo Contínuo. 2008. 136 f. tese (Doutorado em Ciências)-Universidade Federal do Rio Grande do Sul, 2008.

51. KUBICEK, M.; MAREK, M., Computational Methods in Bifurcation Theory and Dissipative Strutures, Springer-Verlag, 1983.

52. CHAMPNEYS, A. R.; DERCOLE, F.; FAIRGRIEVE, T.; KUZNETSOV, Y. PAFFENROTH, R.; SANDSTEDE, B.; WANG, X.; ZHANG, C., AUTO-07P: Continuation and Bifurcation Software for Ordinary Differential Equations,

http://sourceforge.net/projects/auto-07p/files/auto07p/0.8/, Agosto de 2009. 
53. GOVAERTZ, W.; KUZNETSOV, Y. A., MatCont Software de Continuação no MATLAB, http://sourceforg.net/projects/matcont, Abril de 2011.

54. BURDEN, R. L.; FAIRES, J. D., Análise Numérica, Thomson, 2003.

55. WOLF, A.; SWIFT, J. B.; SWINNEY, H. L.; VASTANO, J. A., Determining Lyapunov Exponents From a Time Series, Physica 16D, p. 285-317, 1985.

56. DEITEL, H. M.; DEITEL, P. J., C++ Como Programar, Bookman, 2001.

57. ALBAHADILY, F. N.; SCHELL, M., Observation of sereral different temporal patterns in the oxidation of formic acid at a rotating platinum-disk electrode in on acidic medium, Journal of Electroanalytical Chemistry, v. 308, p. 151-173, 1991.

58. CHEN, S.; LEE, D.; SCHELL, M., Enhancement of the electrochemical oxidation of formic acid. Effects of anion adsorption and variation of rotation rate, Electrochemical Acta, v. 46, p. 3482-3492, 2001.

59. CHEN, S.; LEE, D.; SCHELL, M., Increases in reaction rates and improvement of current-potential characteristics in the electrochemical oxidation of formic acid,

Electrochemical communication, v. 3, p. 81-85, 2001.

60. NAGAO, R.; EPSTEIN, I. R.; GONZALEZ, E. R.; VARELA, H., Temperature (over) compensation in on oscilatory surface reaction, Journal of Physical Chemistry A, v. 112, p. 4617-4624, 2008.

61. BATISTA, B. C.; VARELA, H., Open Circuit Interaction of Formic Acid with Oxidized Pt Surfaces: Experiments, Modeling, and Simulations, Journal of Physical Chemistry C, v. 114, p. 18494-18500, 2010.

62. BOCHETO, E.; BATISTA, B. C.; LIMA, R. B.; VARELA, H., A surface-enhanced infrared absorption spectroscopic (SEIRAS) study of the oscillatory electro-oxidation of methanol on platinum, Journal Electroanalytical Chemistry, v. 642, p. 17-21, 2010.

63. MARTINS, A. L.; BATISTA, B. C.; SITTA, E.; VARELA, H., Oscillatory Instabilities during the Electrocatalytic Oxidation of Methanol on Platinum, Journal Brazilian Chemical Society, v. 19, p. 679-687, 2008.

64. CHEN, S.; SCHELL, M., Bistability and excitability in the electrochemical oxidation of ethanol, Electrochemical Acta, v. 44, p. 4773-4780, 1999.

65. CHEN, S.; SCHELL, M., A comparison of multistability in the electrocatalyzed oxidations of methanol and ethanol in acid and alkaline solutions, Journal Electroanalytical Chemistry, v. 478 , p. $108-117,1999$.

66. KIKUCHI, M.; MUKOUYAMA, Y.; OKAMOTO, H., Chloride ion influencing potential oscillation generated by formaldehyde oxidation, Electrochemical Acta, v. 53, p. 7817-7824, 2008 .

67. OKAMOTO, H.; TANAKA, N.; NAITO, M., Chaotic and periodic potential oscillations in formaldehyde oxidation, Journal of Physical Chemistry A, v. 102, p. 7343-7352, 1998. 
68. PLENGE, F.; VARELA, H.; LÜBKE, M.; KRISCHER, K., Quantitative Modeling of the Oscillatory Electrooxidation of Hydrogen on Pt in the Presence of Poisons, Z. Phys. Chem., v. 217 , p. $365-381,2003$.

69. MARTINS, A. L.; BATISTA, B. C.; SITTA, E.; VARELA, H., Oscillatory Instabilities during the Electrocatalytic Oxidation of Methanol on Platinum, Journal Brazilian Chemical Society, v. 19, p. 679-687, 2008.

70. GASPARD, P.; NICOLIS, G., What Can We Learn from Homoclinic Orbits in Chaos Dynamics?, Journal of Statistical Physics, v. 31, p. 499-518, 1983.

71. FEIGENBAUM, M. J., Quantitative Universality for a Class of Nonlinear Transformations, Journal of Statistical Physics, v. 19, p. 25-52, 1978.

72. GASPARD, P.; KAPRAL, R.; NICOLIS, G., Bifucation Phenomena near Homoclinic Systems: A Two-Parameter Analysis, Journal of Statistical Physics, v. 35, p. 697-727, 1984.

73. GASPARD, P.; WANG, X.-J., Homoclinic Orbtis and Mixed-Mode Oscillations in Far-from-Equilibrium Systems, Journal of Statistical Physics, v. 48, p. 151-199, 1987.

74. WIGGINS, S., Introduction to Applied Nonlinear Dynamical Systems and Chaos, Springer, 2003.

75. GUCKERNHEIMER, J.; HOLMES, P., Nolinear Oscillations, Dynamical Systems, and Bifurcations of Vector Fields (Applied Mathematical Sciences 42), Springer, 2002.

76. ARNEODO, A.; COULLET, P.; TRESSER, C., Oscillators with Chaoitic Behavior: An Illustration of a Theorem by Shil'nikov, Journal of Statistical Physics, v.27, p. 171-182, 1982.

77. GLENDINNING, P.; SPARROW, C., Local and Global Behavior near Homoclinic Orbits, Journal of Statistical Physics, v. 35, p. 645-695, 1984.

78. SPARROW, C., The Lorenz Equations: Bifurcation, Chaos and Strange Attractors, Springer New York, 1982.

79. RÖSSLER, O. E., Continuous Chaos-Four Prototype Equations, Annals New York Academy of Sciences, v. 78, p. 376-392, 1979.

80. KOPER, M. T. M.; GASPARD, P.; SLUYTERS, J. H., Mixed-mode oscillations and incomplete homoclinic scenarios to a saddle focus in the indium/thiocyanate electrochemical oscillator, Journal of Chemical Physics, v. 97, p. 8250-8260, 1992.

81. KOPER, M. T. M.; GASPARD, P., The modeling of mixed-mode and chaotic oscillations in electrochemical systems, Journal of Chemical Physics, v. 96, p. 7797-7813, 1992.

82. DESROCHES, M.; JEFFREY, M. R., Canards and curvature: nonsmooth approximation by pinching, Nonlinearity, v. 24, p. 1655-1682, 2011.

83. DESROCHES, M.; JEFFREY, M. R., Canards and curvature: 'the smallness of $\varepsilon$ ' in slow-fast dynamics, Proceedings of the Roiyal Society A-Mathematical Physical and Engineering Sciences, v. 465, p. 2404-2421, 2011. 
84. FREIRE, J. G.; GALLAS, J. A. C., Stern-Brocot trees in cascades of mixed-mode oscillations and canards in the extended Bonhoeffer-van der Pol and the FitzHugh-Nagumo models of excitable systems, Physics Letters A, v. 375, 1097-1103, 2011.

85. ZHAO, Y.; WANG, S.; VARELA, H.; GAO, Q.; HU, X.; YANG, J.; EPSTEIN, I. R., Spatiotemporal Pattern Fromation in the Oscillatory Electro-Oxidation of Sulfide on a Platinum Disk, The Journal of Physical Chemistry C, v. 115, p. 12965-12971,, 2011.

86. BARRIO, R.; BLESA, F.; SERRANO, S.; SHILNIKOV, A., Global organization of spiral structures in biparameter space of dissipative systems with Shilnikov saddle-foci, Physical Review E, v. 84, p. 035201(1-5), 2011.

87. SWINNEY, H. L., Observations of order and chaos in nonlinear system, Physical D, v. 7, p. 3-15, 1983.

88. ROUX, J. C.; SIMOYI, R. H.; SWINNEY, H. L., Observation of a strange attractor, Physica D, v. 8, p. 257-266, 1983.

89. OTT, W.; YORKE, J. A., When Lyapunov exponents fail to exist, Physical Review E, v. 78 , p. 1-6, 2008.

90. BONATTO, C.; GARREAU, J. C.; Gallas J. A. C., Self-Similarities in the Frequency-Amplitude Space of a Loss-Modulated $\mathrm{CO}_{2}$ Laser, Physical Review Letters, v. 95, p. 143905 (1-4), 2005.

91. FREIRE, J. G.; FIELD, R. J.; GALLAS, J. A. C., Relative abundance and structure of chaotic behavior: The nonpolynomoal Belousov-Zhabotinsky reaction kinetics, The Journal of Chemical Physics, v. 131, p. 044105 (1-8) , 2009.

92. FREIRE, J. G.; BONATTO, C.; CAMARA, C.; GALLAS, J. A. C., Multistability, phase diagrams, and intransitivity in the Lorenz-84 low-order atmospheric circulation model, Chaos, v. 18, p. 033121(1-8), 2008.

93. BONATTO, C.; GALLAS, J. A. C., Accumulation boundaries: codimension-two accumulation of accumulations in phase diagrams of semiconductor lasers, electric circuits, atmospheric and chemical oscillators, Philosophical Transactions of the Royal Society A, v. 366, p. 505-517, 2008.

94. BONATTO, C.; GALLAS, J. A. C.; UEDA, Y., Chaotic phase similarities and recurrences in a damped-driven Duffing oscillator, Physical Review E, v. 77, p. 026217(1-5), 2008.

95. GALLAS, J. A. C., The structure of infinite periodic and chaotic hub cascades in phase diagrams of simple autonomous flows, International Journal of Bifurcation and Chaos, v. 20, p. 197-211, 2010.

96. FREIRE, J. G.; GALLAS, J. A. C., Non-Shilnikov cascades of spikes and hubs in a semiconductor laser with optoelectronic feedback, Physical Review E, v. 82, p. 037202 (1-4), 2010 .

97. FREIRE, J. G.; GALLAS, J. A. C., Stern-Brocot trees in the periodicity of mixed-mode oscillations, Physical Chemistry Chemical Physics, v. 13, p. 12191-12198, 2011. 
98. BATISTA, E. A.;MALPASS, G. R. P.; MOTHEO, A. J.; IWASITA, T., New mechanistic aspects of methanol oxidation, Journal of Electroanalytical Chemistry, v. 571, p. 273-282, 2004.

99. PARSONS, R.; VANDERNOOT, T., The oxidation of small organic molecules: A survey of recent fuel cell related research, Journal Electroanalytical Chemistry, v. 257, p. 9-45, 1988.

100. BATISTA, E. A.;MALPASS, G. R. P.; MOTHEO, A. J.; IWASITA, T., New insight into the pathways of methanol oxidation, Electrochemistry Communications, v. 5, p. 843-846, 2003.

101. DINGWELL, J. B.; NAPOLITANO, D. F.; CHELIDZE, D., A nonlinear approach to tracking slow-time-scale changes in movement kinematics, Journal of Biomechanics, v. 40, p. 1629-1634, 2007.

102. OKAMOTO, H.; TANAKA, N.; NAITO, M., Chaos in the Oxidation of Formaldehyde and/or Methanol, The Journal of Physical Chemistry A, v. 101, p. 8480-8488, 1997.

103. MALINOWSKI, E. D., Factor Analysis in Chemistry, second edition, Wiley-Interscience Publication, 1991.

104. CHELIDZE, D., Indentifying Multidimensional Damage in a Hierarchical Dynamical System, Nonlinear Dynamics, v. 37, p. 307-322, 2004.

105. CHELIDZE, D.; LIU, M., Multidimensional Damage Identification Based on Phase Space Warping: An Experimental Study, Nonlinear Dynamics, v. 46, p. 61-72, 2006.

106. CHELIDZE, D; ZHOU, W., Smooth orthogonal decomposition-based vibration mode identification, Journal of Sound and Vibration, v. 292, p. 461-473, 2006.

107. CHELIZE, D; LIU, M., Dynamical systems approach to fatigue damage identification, Journal of Sound and Vibration, v. 281, p. 887-904, 2005.

108. JOHNSON, R. A.; WICHERN, D. W., Applied Multivariate Statistical Analysis, Prentice-Hall, 1998.

109. NAGAO, R.; SITTA, E.; VARELA, H., Stabilizing Nonstationary Electrochemical Time Series, The Journal of Physical Chemistry C, v. 114, p. 22262-22268, 2010.

110. KENNEL, M.; BROWN, R.; ABARBANEL, H. D. I., Determining embedding dimension for phase-space reconstruction using a geometrical construction, Physical Review A, v. 45, p. 3403-3411, 1992.

111. POLLICOTT, M., Time-delay coordinates and polynomial mappings, Advances in Mathematics, v. 177, p. 280-296, 2003.

112. PAVLOS, G. P.; ATHANASIU, M. A., SVD analysis of the magnetospheric AE index time series and comparison with low-dimensional chaootic dynamics, Nonlinear Processes in Geophysics, v. 8, p. 95-125, 2001. 
113. FRASER, A. M.; SWINNEY, H. L., Independent coordinates for strange attractors from mutual information, Physical Review A, v. 33, p. 1134-1140, 1986.

114. CHELIDZE, D., Nonlinear Dynamics Laboratory, Phase Space Warping, 2002.

Disponível em: <http://mcise.uri.edu/chelidze/nld/>. Acessado em: 19 de Outubro de 2011.

115. KUNIMATSU, K.; HANAWA, H.; UCHIDA, H.; WATANBE, M., Role of adsorbed species in methanol oxidation on $P t$ studied by ATR - FTIRAS combined with linear potencial sweep voltammetry, Journal of Electroanalytical Chemistry, v. 632, 109-119, 2009.

116. TAULER, R., Application of non-linear optimization methods to the estimation of multivariate curve resolution solutions and of their feasible band boundaries in the investigation of two chemical and envirommental simulated data sets, Analytica Chimica Acta, v. 595, p. 289-298, 2007.

117. JAUMOT, J.; GARGALLO, R.; JUAN, A.; TAULER, R., A graphical user-friendly interface for MCR-ALS: a new tool for multivariate curve resolutoin in MATLAB, Chemometrics and Intelligent Laboratory Systems, v. 76, p. 101-110, 2005.

118. JAOUMOT, J.; MARCHAN, V.; GARGALLO, R.; GRANDAS, A.; TAULER, R., Multivariate Curve Resolution Applied to the Analysis and Resolution of Two-Dimensional $\left[{ }^{1} H,{ }^{15} N\right]$ NMR Reaction Spectra, Analytical Chemistry, v. 76, p. 7094-7101, 2004.

119. JUAN, A.; MAEDER, M.; MARTINEZ, M.; TAULER, R., Combining hard- and soft-modelling to solve kinetic problems, Chemometrics and Intelligent Laboratory Systems, v. 54, p. 123-141, 2000.

120. TAULER, R., Institute of Environmental Assessment, Programs and utilities

(MATLAB). Disponível em: <http://www.cid.csic.es/homes/rtaqam/>. Acessado em: 20 de Outubro de 2011. 


\section{Curriculum Vitae}

\section{Dados Pessoais}

Nome: Melke Augusto do Nascimento

Nascimento: 31 de Janeiro de 1979 - Natal/RN - Brasil

Filiação: João Augusto do Nascimento e Iracema Coelho do Nascimento

\section{Formação Acadêmica/Titulação}

(2007-2011) Doutorado em Química com área de concentração em FísicoQuímica

Universidade de São Paulo, USP, São Paulo, Brasil

Título: Instabilidades Cinéticas em Sistemas Eletroquímicos: Uma Contribuição Teórica

Ano de obtenção: 2011

Orientador: Hamilton Brandão Varela Albuquerque

Bolsista do(a): Fundação de Amparo à Pesquisa no Estado de São Paulo (FAPESP)

(2005-2007) Mestrado em Química

Universidade Federal do Rio Grande do Norte, UFRN, Natal, Brasil.

Título: Aplicação de tecnicas multivariada e correlação $2 \mathrm{D}$ em espectros de absorção de raio $x$

Ano de obtenção: 2007

Orientador: Renato Canha Ambrosio

Bolsista do(a): Coordenação de Aperfeiçoamento de Pessoal de Nível Superior

(1999-2004) Graduação em Química licenciatura

Universidade Federal do Rio Grande do Norte, UFRN, Natal, Brasil 


\section{Publicações}

1. M. A. Nascimento, J. A. C. Gallas, H. Varela; Self-organized distribution of periodicity and chaos in an electrochemical oscillator; Physical Chemistry Chemical Physics, v. 13, p. 441-446, 2011. (Capa da revista)

2. S. Sauerbrei, M. A. Nascimento, M. Eiswirth, and H. Varela, Mechanism and model of the oscillatory electro-oxidation of methanol, The Journal of Chemical Physics, v. 132, p. 154901(1-10), 2010.

3. Elton Sitta, Melke A. Nascimentoa and Hamilton Varela, Complex kinetics, high frequency oscillations and temperature compensation in the electro-oxidation of ethylene glycol on platinum, Physical Chemistry Chemical Physics, v. 12, p. 15195-15206, 2010.

\section{Congressos}

1. Nascimento, Melke A.; Varela, H.; Tremiliosi-Filho, G. Modelagem e Simulação da Dinâmica Oscilatória em Reações Eletrocatalíticas. 2009. (Apresentação de Trabalho/Congresso). Palavras-chave: Modelagem; Eletroquímica; Dinâmica Oscilatória. Referências adicionais: Brasil/Português; Local: CE-Brasil; Cidade: Fortaleza-CE-Brasil; Evento: XVII Simósio Brasileiro de Eletroquímica e eletroanalítica. (Apresentação oral e pôster)

2. $\quad 33^{\circ}$ Reunião Anual da Sociedade Brasileira de Química, período de 28 a 31 de maio de 2010. Titulo do trabalho apresentado: Diagramas de fase de alta resolução da dinâmica oscilatória em modo eletroquímico, autoria de Nascimento, M.A.; Varela, H. (Apresentação oral e pôster)

3. Dynamics Days South America 2010 international Conference on Chaos and Nonlinear Dynamics, in São José dos Campos, SP, Brazil, from July 26th to 30th, 2010, presenting the work entitled High-resolution phase diagrams of a generic electrochemical oscillator. (pôster) 